\title{
Dynamical wetting transitions: liquid film deposition and air entrainment
}

Tak Shing Chan 
Dynamical wetting transitions: liquid film deposition and air entrainment

Tak Shing Chan 


\section{Samenstelling promotiecommissie:}

Prof. dr. Leen van Wijngaarden (voorzitter) Prof. dr. Detlef Lohse (promotor)

Dr. Jacco H. Snoeijer (assistant promotor)

Prof. dr. Bruno Andreotti

Prof. dr. Jens Eggers

Mr. Michel Riepen

Prof. dr. Paul Kelly

Prof. dr. Serge Lemay
Universiteit Twente

Universiteit Twente

Universiteit Twente

ESPCI, Paris

University of Bristol

ASML

Universiteit Twente

Universiteit Twente

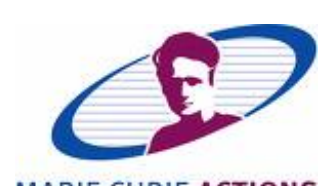

MARIE CURIE ACTIONS
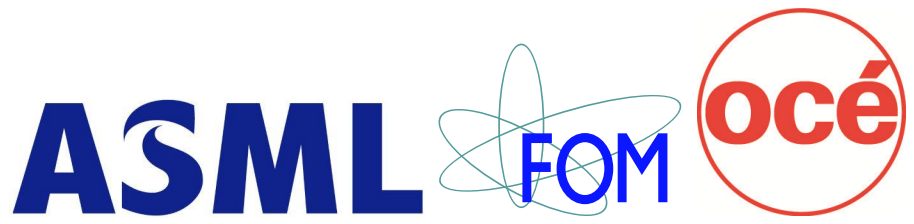

The work in this thesis was carried out at the Physics of Fluids group of the Faculty of

Science and Technology of the University of Twente. The research leading to these results has received funding from the [European Community's] Seventh Framework Programme ([FP7/2007-2013] under grant agreement $n^{o} 215723$. This work is also part of the research programme 'Contact Line Control during Wetting and Dewetting' (CLC) of the Stichting voor Fundamenteel Onderzoek der Materie (FOM), which is financially supported by the Nederlandse Organisatie voor Wetenschappelijk Onderzoek (NWO), ASML and Océ.

Publisher:

Tak Shing Chan, Physics of Fluids, University of Twente, P.O. Box 217, 7500 AE Enschede, The Netherlands pof.tnw.utwente.nl

(C) Tak Shing Chan, Enschede, The Netherlands 2012

No part of this work may be reproduced by print photocopy or any other means without the permission in writing from the publisher

ISBN: 978-90-365-3387-4 


\section{DYNAMICAL WETTING TRANSITIONS: LIQUID FILM DEPOSITION AND AIR ENTRAINMENT}

\section{PROEFSCHRIFT}

ter verkrijging van

de graad van doctor aan de Universiteit Twente,

op gezag van de rector magnificus,

Prof. dr. H. Brinksma,

volgens besluit van het College voor Promoties in het openbaar te verdedigen

op donderdag 30 Augustus 2012 om 16.45 uur

$$
\text { door }
$$

Tak Shing Chan

geboren op $6^{\text {th }}$ February 1982

te GuangDong, China 
Dit proefschrift is goedgekeurd door de promotor:

Prof. dr. rer. nat. Detlef Lohse

en de assistent-promotor:

Dr. Jacco H. Snoeijer 


\section{Contents}

1 Introduction 1

1.1 Moving contact lines . . . . . . . . . . . . . . . 1

1.2 Hydrodynamic challenges . . . . . . . . . . . . . . 3

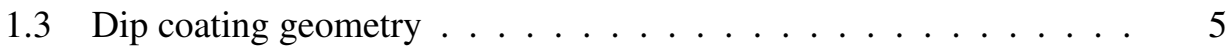

1.4 Guide through the thesis $\ldots \ldots \ldots \ldots$. . . . . . . . . 11

2 Theory of the forced wetting transition 13

2.1 Introduction . . . . . . . . . . . . . . . . . . . . . 13

2.2 Lower branch and critical speed . . . . . . . . . . . . . 17

2.3 Upper branch and bifurcation diagram . . . . . . . . . . . . . 20

2.4 Discussion . . . . . . . . . . . . . . . . . . . . 26

3 Maximum speed of dewetting on a fiber 29

3.1 Introduction . . . . . . . . . . . . . . . . . . . . . . . . 29

3.2 Asymptotic analysis . . . . . . . . . . . . . . 32

3.3 Numerical solution . . . . . . . . . . . . . . . . . . . 40

3.4 Discussion . . . . . . . . . . . . . . . . . . . 45

4 Air Entrainment by Viscous Contact Lines 49

4.1 Introduction . . . . . . . . . . . . . . . . . . . . 49

4.2 Experimental setup and results $\ldots \ldots \ldots \ldots \ldots$

4.3 Interpretation and discussion . . . . . . . . . . . . 55

4.4 Supplementary Material . . . . . . . . . . . . . . . . . . . 57

5 Hydrodynamics of air entrainment by moving contact lines 67

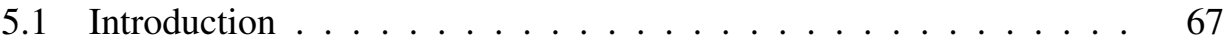

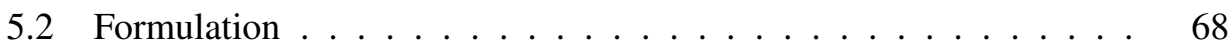

5.3 Methods . . . . . . . . . . . . . . . . 71

5.4 Comparing the lubrication model and Lattice Boltzmann . . . . . 77

5.5 Maximum speeds and transition to air entrainment . . . . . . . . . 81 


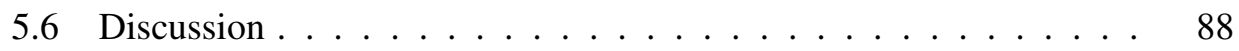

6 Withdrawing a plate from a liquid with small viscosity: effect of inertia 91

6.1 Introduction . . . . . . . . . . . . . . . 91

6.2 Lubrication equation including inertia effect . . . . . . . . . 92

6.3 Results . . . . . . . . . . . . . . . . . . . . . . 97

6.4 Discussion . . . . . . . . . . . . . . . . . . . . . . . 99

$\begin{array}{lll}7 & \text { Summary and Outlook } & 103\end{array}$

$\begin{array}{ll}\text { Samenvatting } & 115\end{array}$

$\begin{array}{ll}\text { Acknowledgements } & 119\end{array}$

$\begin{array}{ll}\text { About the author } & 123\end{array}$ 


\section{1 \\ Introduction}

\subsection{Moving contact lines}

When you see a water drop moving on a leaf or a window, you might not realize that this very common phenomenon is a challenging fluid mechanical problem, involving physics from molecular to macroscopic scales. The behavior of the moving drop is determined by the dynamics of the contact line, the boundary where two immiscible fluids (liquid/gas or liquid/liquid) and a solid meet. One notices that a moving drop appears round at the front, and thinner and sharper at the rear (see Fig. 1.1). Interestingly, detailed experimental studies found that the contact line at the rear develops a corner and this corner becomes sharper as the drop speed increases [1]. Eventually small droplets are released at the tip of the corner if speed is too fast. This instability demonstrates a remarkable property of moving contact lines: the contact line cannot move beyond a maximum speed $U_{c}$ relative to the solid surface $[2,3]$. If one imposes a speed beyond $U_{c}$, there will be a dynamical wetting transition. Like in the moving drop case, the contact line at the rear cannot catch up the speed of the main drop body, thus the liquid at the tip is detached.

Contact line motion is very important in many industrial processes. One example is in Immersion Lithography $[4,5]$, where light is directed through a lens on a substrate to construct circuit patterns. A portion of liquid of high refractive index (usually water) is injected between the lens and the substrate and maintained there 


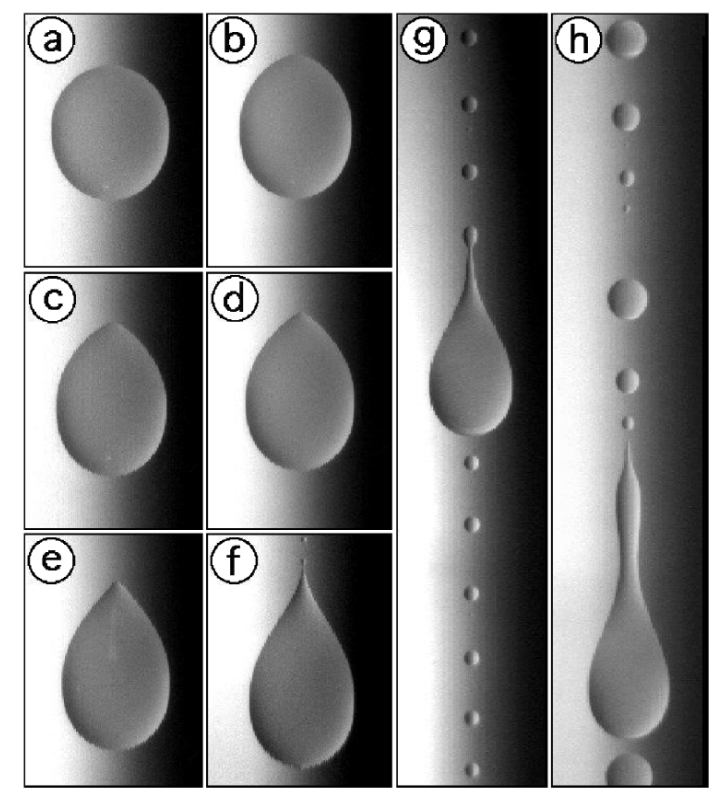

Figure 1.1: Drop running down on an inclined plane at different speeds. Drop speed increases from (a) to (h). (a)-(e) The running drop appears round at the front and sharper at the rear. (f)-(h) Droplets are emitted at the tail of the drop. Figures taken from Podgorski et al. [1].

in order to increase the optical resolutions, see Fig. 1.2a. For example, resolution of $\sim 40 \mathrm{~nm}$ can be achieved when using pure water as the working liquid. Since the substrate cannot be completely covered by the lens, the patterns are not printed on the whole substrate at once. After some patterns have been printed on one region of the substrate, the substrate translates so that an unprinted region is under the cover of the lens. During the translation of the substrate, the contact line is moving relative to the substrate. The problem is that, when the substrate is moving too fast, droplets are emitted at the receding part of the liquid similar to what happens in the moving drop case, see Fig. 1.2b [4]. Similarly, at much higher speed, air bubbles are observed in the advancing part, suggesting entrainment of air at the advancing contact line. All these instabilities could introduce defects to the printed patterns. From an industrial perspective, it is important to push the substrate speed in order to enhance the production rate and lower the cost while maintaining high quality of their products at the same time. For this it is crucial to understand how to optimize the critical speeds. In other words, what are the relevant physical parameters (e.g. wettability of the sur- 
(a)

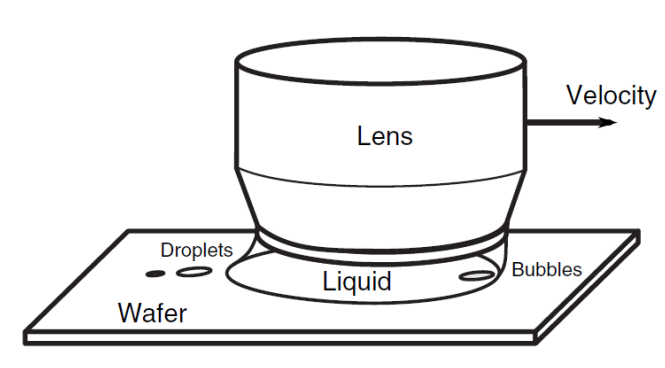

(b)
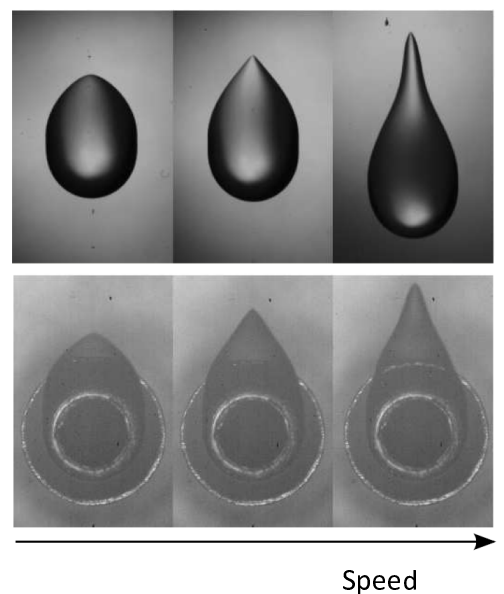

Figure 1.2: (a) Sketch of an Immersion Lithography setup. Small droplets and bubbles appear when the speed is too fast, due to a dynamical wetting transition. (b) Top images are shapes of sliding drops at different speeds as in Fig. 1.1. Bottom images are shapes of water for different speeds of substrate in a setup used in [4] to mimic the Immersion Lithography system. The shapes of the liquids in these two different setups are very similar. Figures taken from Winkels et al.[4].

face, liquid viscosity, surface tension) for the critical speeds of dynamical wetting transitions? How do the critical speeds depend on those parameters? Moreover, the difference in critical speeds of the receding and the advancing parts implies different underlying mechanisms. Why is there a difference? To what extent are the properties of the air relevant for the bubble formation? This thesis therefore addresses the fundamentals of "dynamical wetting transitions".

\subsection{Hydrodynamic challenges}

The fluid mechanical description of contact line motion is challenging for various reasons. Firstly, it has been known since Huh and Scriven [6] pointed out in 1971 that, the shear stress $\tau_{s}$ goes to infinity when approaching the contact line if a no-slip boundary condition is imposed on the liquid/solid interface. This can be understood by a simple argument. Suppose a liquid drop is moving with typical speed $U$. If we look at the tail of the drop, then $\tau_{s} \sim \eta U / h$, where $\eta$ is the liquid viscosity and $h$ the height of the interface, see Fig. 1.3a. When approaching the contact line, $h \rightarrow 0$, then 

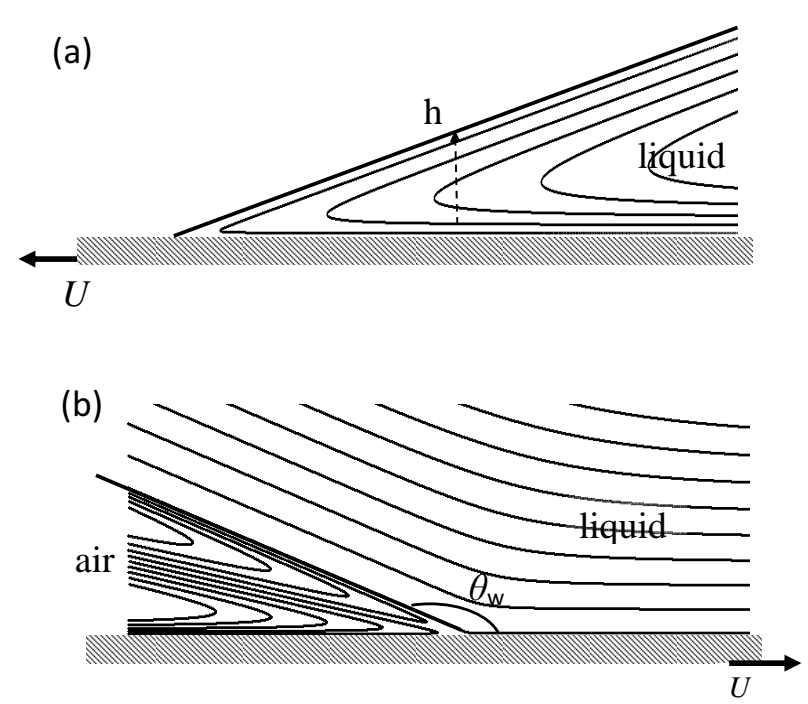

Figure 1.3: (a) Streamlines in a wedge geometry of one-phase flow when a plate is moving with speed $U$. (b) Streamlines in a wedge geometry of two-phase flow when a plate is moving from the air to the liquid (exact solutions by Huh \& Scriven [6]). Streamlines in the air are very dense when the wedge angle $\theta_{w}$ is close to $\pi$.

$\tau_{s} \rightarrow \infty$. This means no drop could move if continuum hydrodynamics with no-slip boundary condition remained valid at the region very close to the contact line. Hence a microscopic mechanism that regularizes the singular tendency at the contact line is required. Many models have been proposed since then that includes precursor film [7], diffusion across the interface [8], Navier-slip condition [6] and others. For an overview we refer the reader to [9]. No matter which model is used, a microscopic length scale has to be introduced to the problem because of the molecular origin of the regularization. This means the typical length scale near the contact line is of order $1 \mathrm{~nm}-10 \mathrm{~nm}$.

Above $10 \mathrm{~nm}$ from the contact line, classical hydrodynamics is valid for the description of the flow in the fluids and the meniscus is determined by the balance between the viscous force and the capillary force [9]. It is natural then to define a dimensionless speed, the capillary number

$$
\mathrm{Ca} \equiv \eta U / \gamma,
$$

where $\gamma$ is the liquid-gas surface tension. As we move further away from the contact line, reaching macroscopic scales, the viscous effect diminishes. At large scales, 
the shape of the interface is determined by the surface tension and the body force, e.g. gravity. In this region, we have macroscopic length scale, e.g. capillary length of order millimeters or the size of the drop. We thus see that there are different length scales involved, characterized by different physical mechanisms. This multiscale property makes the problem difficult to solve. One difficulty one may think of immediately is that large amount of computation resource in numerical calculations is required to resolve the small scales near the contact line, as also a well-known difficulty in study of turbulence.

A second difficulty of the moving contact line problem is the following. In most of the situations one aims to determine the shape of the fluid-fluid interface and how the interface shape evolves. The interface profiles can be computed by solving normal stress condition at the interface (the Young-Laplace equation) which relates the local curvature $\kappa$ of the interface to the normal stress difference across the interface $\delta \tau_{n}$ :

$$
\gamma \kappa=\delta \tau_{n}
$$

To evaluate $\delta \tau_{n}$, one needs to determine the velocity fields in the fluids, but these depend on the shape of the interface. Solving this coupled problem is very challenging.

Thirdly, the standard theoretical tool to deal with problems of flow in thin film is the so-called lubrication theory [10]. When applying it to moving contact line problems, it requires (i) the local slope of the interface to be small, i.e. $h^{\prime} \ll 1$ and (ii) the surrounding air viscosity to be negligible. The first condition usually means that the equilibrium contact angle $\theta_{e}$ has to be small. Experimentally this condition can be fulfilled by properly choosing the material of the substrate and the working fluids. The second condition is more subtle in particular when air entrainment occurs at the advancing contact line. In that case, the local angle of the interface measured in the air is small. One can imagine that the shear stress in the air could be significant since the air is forced to flow in a very confined region. To demonstrate this, we plot the streamlines of flow in a wedge of small angle in air in Fig. 1.3b. We clearly see that the streamlines are extremely dense in the air. This means velocity gradients are large, and thus shear stresses could be significant even though the air viscosity is small. Hence to study air entrainment, one has to consider both the flows in the liquid and the air.

\subsection{Dip coating geometry}

In this thesis, we wish to reveal the nature of dynamical wetting transitions for receding and advancing contact lines. We focus on the common setup of dip coating, a plate of partially wetting surface $\left(\theta_{e}>0\right)$ being withdrawn from, or plunged into, a 
(a)

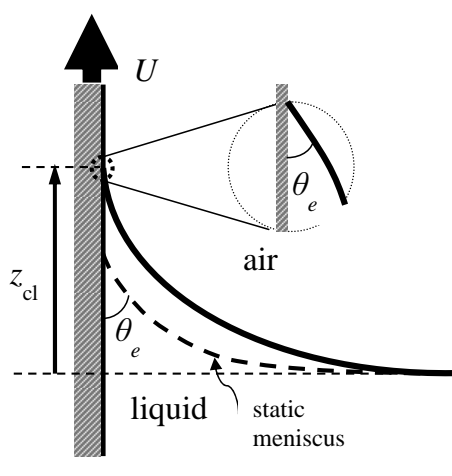

(b)

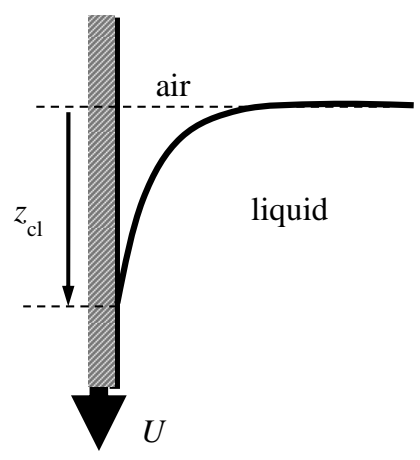

Figure 1.4: Schematic of the dip coating geometry. (a) When $U=0$, the meniscus touches the wall at an static contact angle $\theta_{e}$ (dashed curve). When the plate is being withdrawn from the reservoir, $U \neq 0$, the contact line equilibrates above the static equilibrium position (solid curve). Zoom: In this study, we assume the microscopic contact angle at the contact line remains the same as the static contact angle $\theta_{e}$. (b) When the plate is plunged, the contact line position $z_{\mathrm{cl}}$ falls below its static equilibrium value.

bath of liquid with speed $U$ as shown in Fig. 1.4. The advantage of this geometry with respect to sliding drops or immersion lithography (Fig. 1.2) is that the flow is twodimensional so that the description of the problem is largely simplified. This allows for a detailed study of the physical mechanisms underlying the dynamical wettting transitions.

\subsubsection{Receding contact lines: film deposition}

The dip coating geometry has been studied extensively recently, in particular the receding contact line problem, in which case the plate is withdrawn [3,11-15]. If the plate is not moving, $U=0$, there is no flow in the fluids, and the interface equilibrates to a static shape due to balance between capillarity and gravity. The interface makes an equilibrium angle $\theta_{e}$ with the solid as a result of intermolecular interaction between the three phases at the contact line. When the plate is moving, the viscous drag generated by the moving plate deforms the fluid interface gradually. After a transient time, the contact line position $z_{\mathrm{cl}}$ relaxes to a steady position above its static equilibrium value as long as $U$ is below a critical value $U_{c}$, see Fig 1.4a. For $U>U_{c}$, a dynamical wetting transition occurs during which a liquid film will be 
deposited on the solid surface.

Using lubrication theory [13], one can compute the steady menisci up to the critical capillary number, $\mathrm{Ca}_{\mathrm{c}} \equiv \eta U_{c} / \gamma$. To characterize the solutions, the meniscus rise $z_{\mathrm{cl}}$ is plotted as function of Ca in Fig. 1.5a (solid curve) [14]. We see that $z_{\mathrm{cl}}$ increases from the static equilibrium position as $\mathrm{Ca}$ increases. After reaching $\mathrm{Ca}_{\mathrm{c}}$ (point (ii) of the solid curve, we refer those solutions before as lower branch), the curve then turns back to $\mathrm{Ca}<\mathrm{Ca}_{\mathrm{c}}$ but with solutions of increasing $z_{\mathrm{cl}}$ (these solutions are called upper branch). Further upwards, we observe a series of bifurcations. The shapes of the menisci at different positions of the bifurcation diagram are plotted in Fig. 1.5b. For solutions above point (iii) indicated in the bifurcation diagram, the menisci develop a dimple and above it a ridge-like shape. This ridge grows longer and longer as we move further upward in the bifurcation diagram $[12,16]$. Interestingly, in the limit of $z_{c l} \rightarrow \infty$ corresponding to $\mathrm{Ca}=\mathrm{Ca}^{*}$, a film solution referred as "thick film" can be found, see Fig. 1.5c [15]. Again, the thick film solution matches to the bath through a dimple in between. We should emphasize that the thick film solution is different qualitatively from the classical Landau-Levich (LLD) film [17].

The lubrication calculations of the lower branch of the steady solutions have been well verified by experiments for $\mathrm{Ca}<\mathrm{Ca}^{*}$ [14]. For $\mathrm{Ca}^{*}<\mathrm{Ca}<\mathrm{Ca}_{\mathrm{c}}$, the scenario is more complex. Although steady solutions can still be obtained from the lubrication theory, surprisingly, detailed experimental studies found that the dynamical wetting transition starts at $\mathrm{Ca}^{*}$ instead of $\mathrm{Ca}_{c}[12,14]$. Why the critical speed $\mathrm{Ca}_{\mathrm{c}}$ is avoided remains an open question.

Fig. 1.6 shows a photo of an experiment during dynamical wetting transition, we see a ridge is formed right behind the contact line and is propagating upward [12]. We can also observe that the liquid is not entrained at the edge of the plate which has non-zero curvature. From this one may expect the critical speed at the edge of the plate to be higher. Moreover, contrary to the plate case, when withdrawing a fiber of small radius, Sedev and Petrov [18] found transition occurs at $\mathrm{Ca}=\mathrm{Ca}_{\mathrm{c}}$. This raises the question of how the geometry of the flow can influence the dynamical wetting transition.

\subsubsection{Advancing contact lines: air entrainment}

The reverse process of withdrawing a plate is plunging a plate into a liquid reservoir, see Fig. 1.4b. In this case, we expect air entrainment to occur above a critical velocity. This means the angle in the air gets smaller as Ca increases. Near the dynamical wetting transition (the wedge angle $\theta_{w}$ close to $\pi$ ), we argued previously that air viscosity could play a role since air is forced to flow in a very confined region, see Fig. $1.3 \mathrm{~b}$. So an interesting question to ask is: is air viscosity $\eta_{g}$ really relevant for 


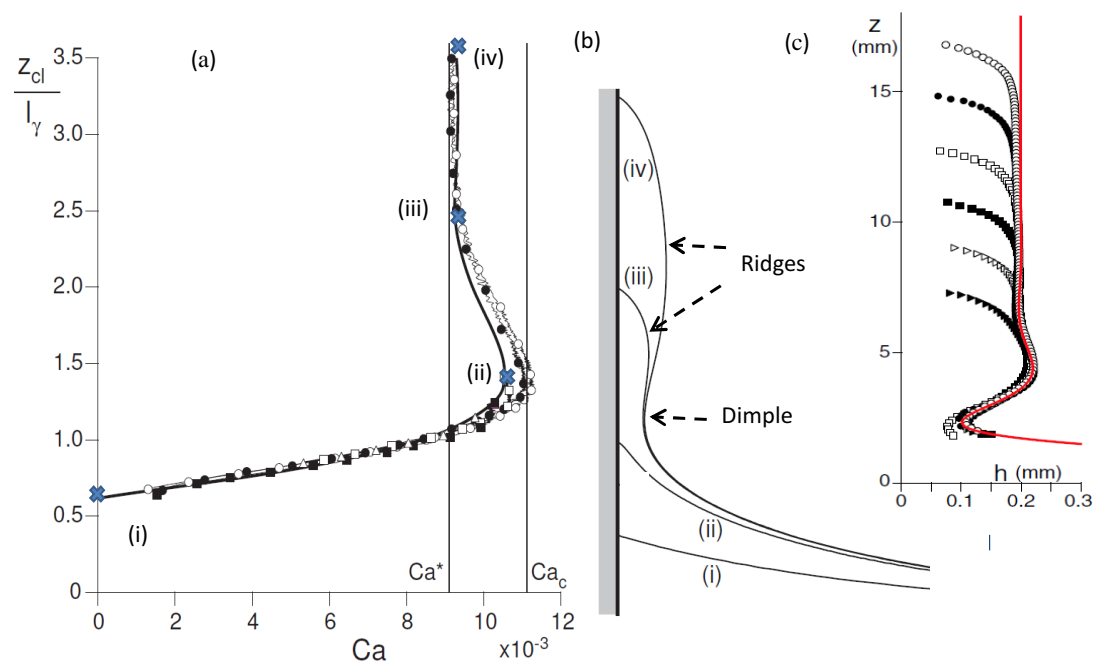

Figure 1.5: (a) Meniscus rise $z_{\mathrm{cl}}$ normalized by the capillary length $\ell_{\gamma}$ versus Ca. Solid curve: steady solutions calculated by lubrication theory, point (ii) corresponds to the maximum, or critical capillary number $\mathrm{Ca}_{\mathrm{c}}$. Symbols: experimental results obtained during dynamical wetting transition, here the capillary number is defined by the contact line speed with respect to the plate instead of plate speed. For details, we refer to Delon et al. [14]. (b) Stationary interface profiles at different positions of the bifurcation diagram in (a). Note that all the curves have the same bath position. Figures taken from Delon et al. [14]. (c) Red curve: Thick film solution calculated by lubrication equation. Symbols are evolution of the interface observed in experiment during the dynamical wetting transition. Figure taken from Snoeijer et al. [15].

the advancing motion of contact lines?

A very similar scenario for air entrainment without contact line motion [19] has been observed for the case where a viscous liquid impacts a reservoir, as in Fig. 1.7. In this study, a horizontal cylinder partially immersed in a reservoir is rotating with constant speed, thus dragging the liquid on the left out from the bath. This liquid flows along the surface of the cylinder and hits the liquid bath on the right. It has been observed that the air-liquid interface forms a cusp shape at the region of impact (Fig. 1.7a). Above a critical speed, air is entrained (Fig. 1.7b). Lorenceau et al. [19] found that the critical capillary number of air entrainment $\mathrm{Ca}_{c}$ depends logarithmically on 


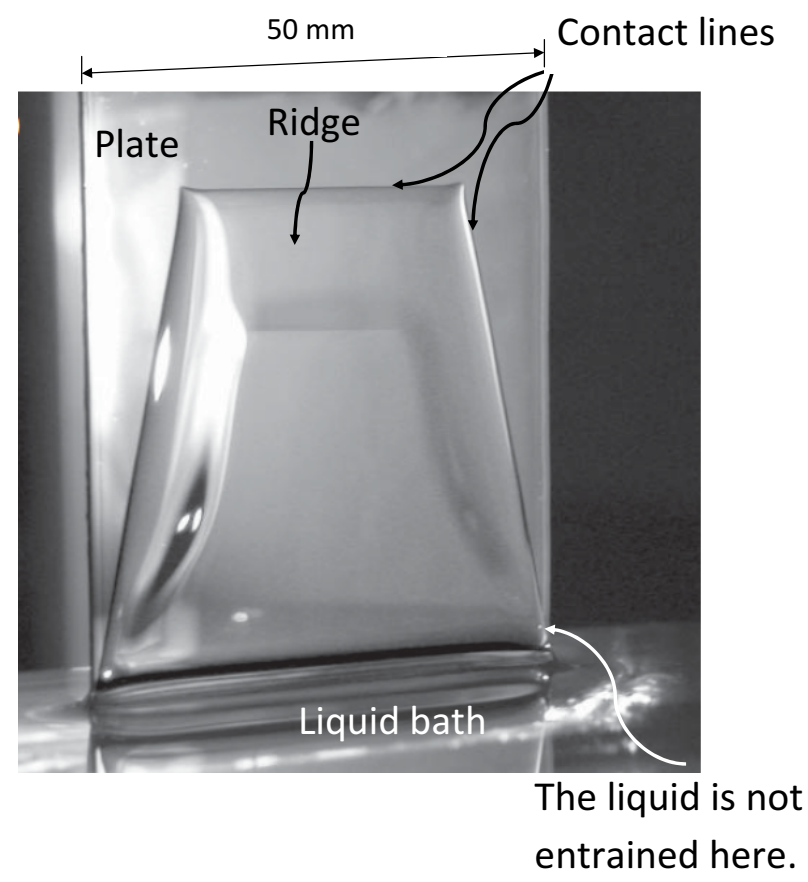

Figure 1.6: Photograph of the withdrawing plate experiment using a very viscous silicone oil (viscosity $=4.95 \mathrm{~Pa} \cdot \mathrm{s}$ ). A ridge is formed right behind the contact line and propagating upward during dynamical wetting transition. Note that while the liquid is entrained at the central region, the contact line has remained still at the edge of the wafer. Figure taken from Snoeijer et al. [12].

the ratio between the air viscosity and the liquid viscosity $\left(R \equiv \eta_{g} / \eta\right)$,

$$
\mathrm{Ca}_{\mathrm{c}} \equiv \frac{\eta_{\ell} U_{c}}{\gamma} \sim-\ln R,
$$

consistent with the prediction by Eggers's theory [20]. Does a similar result hold for the plunging plate case in which there is a contact line motion? Using matched asymptotic expansions, Cox extended the study for one phase to two-phase moving contact line problem in a general geometry [21]. He found results similar to the liquid impact case: a weak logarithmic dependence of the critical capillary number on the viscosity ratio $R$. However, as acknowledged already by Cox, the assumptions of the model break down when $\mathrm{Ca}$ is close to $\mathrm{Ca}_{\mathrm{c}}$. So what do we observe if we investigate the problem experimentally, for example, in the dip-coating geometry? 

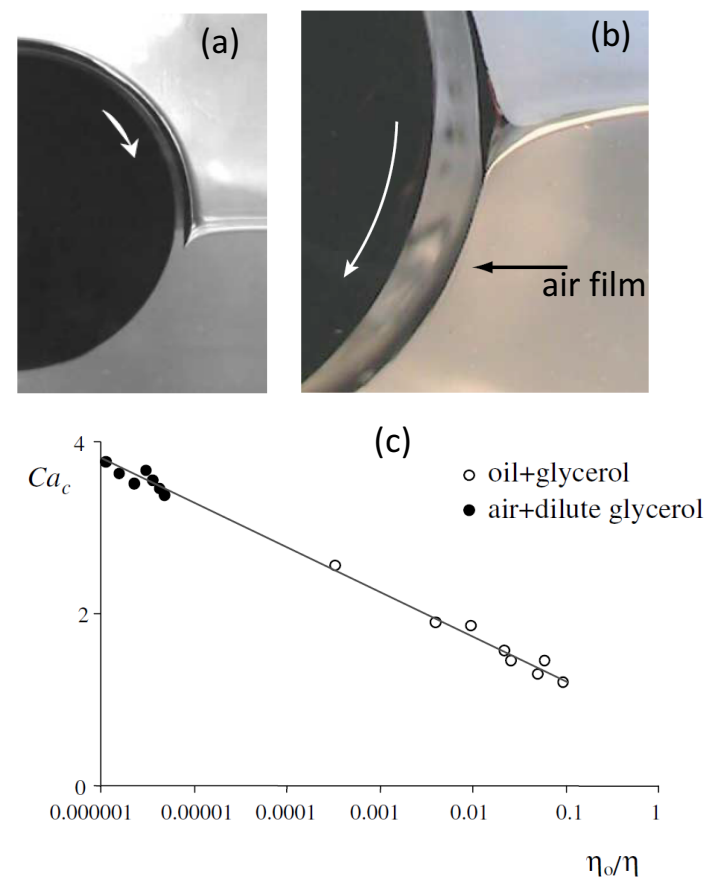

Figure 1.7: (a) A rotating horizontal cylinder is partially immersed in a bath of liquid. The liquid on the left is dragged out from the bath and hits the liquid bath on the right, thus the interface is deformed at the region of impact. (b) Air film (black line) is entrained to the liquid when the cylinder is rotating faster than the critical speed. (c) Critical speed of air entrainment $\mathrm{Ca}_{\mathrm{c}}$ as function of viscosity ratio (on logarithmic scale) between the upper fluid $\left(\eta_{o}\right)$ and the bottom fluid $(\eta)$. Figures taken from Lorenceau et al. [19].

More generally, the role of air has recently become a subject of discussion in a wide context of problems. When impacting a drop on a substrate, it was found that splashing can be suppressed completely by reducing the air pressure [22]. Remarkably, in experiments of plunging a tape into a liquid bath, Benkreira et al. [23] observed that the critical speeds of air entrainment can be increased by depressurizing the gas, see Fig. 1.8. Yet, a pressure reduction does not affect the dynamical viscosity of the air. It thus remains unclear how the air plays a role for all these phenomena. 


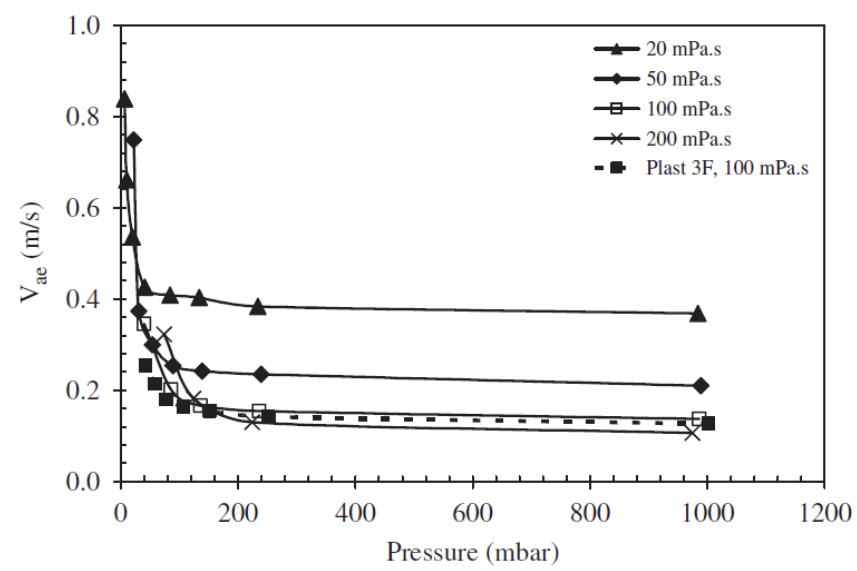

Figure 1.8: Experimental results of plunging a tape into a liquid bath: Critical speed of air entrainment $V_{a e}$ as function of air pressure. Different symbols correspond to different liquid viscosities. $V_{a e}$ is enhanced by depressurizing the air. Figure taken from Benkreira et al. [23].

\subsection{Guide through the thesis}

In this thesis we investigate dynamical wetting transitions in the dip-coating geometry and address the questions raised above. In Chapter 2 and 3, we investigate liquid film deposition by receding contact lines, while Chapter 4 and 5 address air entrainment by advancing contact lines.

The withdrawing plate problem can be investigated by numerically solving the lubrication equation discussed above. On the other hand, the existence of two separated length scales (from nanometer to millimeter) allows for analysis using matched asymptotic expansion. This method has been applied to study the withdrawing plate problem by Eggers $[3,11]$ and provides the lower branch solutions of the bifurcation diagram and the critical capillary number. In Chapter 2, we will implement this method to study the upper branch solutions and show that the bifurcation at the critical point is a saddle-node type.

To understand how the geometry of the wetting flow influences the dynamical wetting transition, we investigate a problem of withdrawing a fiber from a liquid reservoir in Chapter 3. We calculate the critical speeds and the bifurcation diagrams for different values of fiber radius. We will show that the critical speed decreases with the radius of the fiber. Strikingly, we will demonstrate that the bifurcation diagram for small fiber radius differs qualitatively from that for large fiber radius. 
To unravel the role of the air, we experimentally study a plate being plunged into a reservoir of silicon oil of different viscosities in Chapter 4. We will show that the air viscosity plays an important role in air entrainment which is not expected from the classical viewpoint of wetting problems.

In Chapter 5, we will generalize the lubrication model to situations of two-phase flow. This will allow us to investigate air entrainment in the presence of a moving contact line. In particular, we investigate the dynamical wetting transition of air entrainment. The results of the two-phase hydrodynamic theory will be compared with Lattice Boltzmann simulations.

In Chapters 2 - 5, we focus on the regime of small Reynolds number $R e \equiv$ $\rho U L / \eta$, thus the inertia effect is not taken into consideration. However, some very common fluids (e.g. water) we encounter in environment and industrial applications have a relatively low viscosity. For example, in Immersion Lithography, the fluid used in between the lens and the substrate is water which has viscosity $\eta$ around $10^{-3} \mathrm{mPa} \cdot \mathrm{s}$ and density $\rho=10^{3} \mathrm{~kg} / \mathrm{m}^{3}$ at room temperature. If we take the typical speed of flow $U$ as $0.1 \mathrm{~m} / \mathrm{s}$ and the distance between the lens and the substrate $L$ as $10^{-4} \mathrm{~m}$, then $R e=10$. In Chapter 6, we will consider the situation in which $R e$ is not so small. Again we consider a dip-coating geometry. We will develop a lubricationtype model for one-phase flow with the inertial term taken into account. 


\section{2 \\ Theory of the forced wetting transition}

We consider a solid plate being withdrawn from a bath of liquid which it does not wet. At low speeds, the meniscus rises below a moving contact line, leaving the rest of the plate dry. At a critical speed of withdrawal, this solution bifurcates into another branch via a saddle-node bifurcation: two branches exist below the critical speed, the lower branch is stable, the upper branch is unstable. The upper branch eventually leads to a solution corresponding to film deposition. We add the local analysis of the upper branch of the bifurcation to a previous analysis of the lower branch. We thus provide a complete description of the dynamical wetting transition in terms of matched asymptotic expansions.

\subsection{Introduction}

Consider a partially wetting solid plate (with microscopic contact angle $\theta_{\mathrm{e}}$ ), being withdrawn from a liquid reservoir, as illustrated in Fig. 2.1. Depending on the speed of withdrawal $U$, two scenarios can occur. If $U$ is above a certain threshold value $U_{c}$, a liquid film is deposited on the solid surface $[2,9,12,15,24]$. This principle is commonly used in the coating industry $[25,26]$. On the other hand, if $U<U_{c}$ , an initially dry solid surface will remain dry, but the contact line position $z_{\mathrm{cl}}$ (see

${ }^{*}$ Published as: T.S. Chan, J.H. Snoeijer, and J. Eggers, Theory of the forced wetting transition, Phys. Fluids 24, 072104 (2012). 


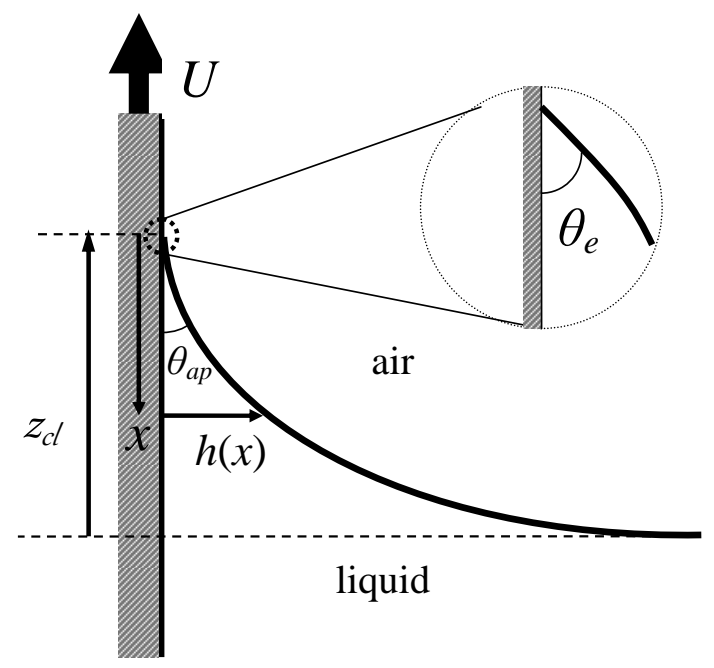

Figure 2.1: Schematic diagram of a plate being withdrawn from a viscous liquid reservoir. The interface shape is $h(x)$, where $x$ is measured relative to the contact line position: $x=z_{c l}-z$. From a large scale, the interface meets the wall with an apparent contact angle $\theta_{a p}$. Near the contact line, the interface is highly curved, and one recovers the equilibrium contact angle $\theta_{\mathrm{e}}$ at the contact line.

Fig. 2.1) rises above its equilibrium value. The critical speed is set by a balance between the liquid-gas surface tension $\gamma$ and viscous forces [21,27], which are proportional to the liquid viscosity $\eta$. As a result, the critical speed is controlled by the dimensionless capillary number $\mathrm{Ca} \equiv \eta U / \gamma$. For simplicity, we restrict ourselves to the most frequently used geometry of a plate being withdrawn vertically.

The bifurcation between wetted and dry states can be understood by considering the solution curve shown in Fig. 2.2a, which plots $z_{\mathrm{cl}}$ as function of the plate speed $[13,28]$. Here and in the following, all lengths are scaled by the the capillary length $\ell_{c} \equiv \sqrt{\gamma / \rho g}$, where $\rho$ is the fluid density and $g$ the acceleration of gravity. Stationary solutions have been computed numerically using a modified lubrication theory [29], which remains valid for arbitrary interface slopes. The only restriction on its validity, when compared to the full viscous fluid equations, is that of small $\mathrm{Ca}$. To model the contact line motion, we introduce a microscopic slip length $\lambda_{s}$, which is necessary for the contact line to be able to move $[6,9]$. On the scale of $\lambda_{s}$, the interface makes a finite contact angle with the solid, which we take to be the equilibrium contact angle. More details of the modeling will be discussed below. 

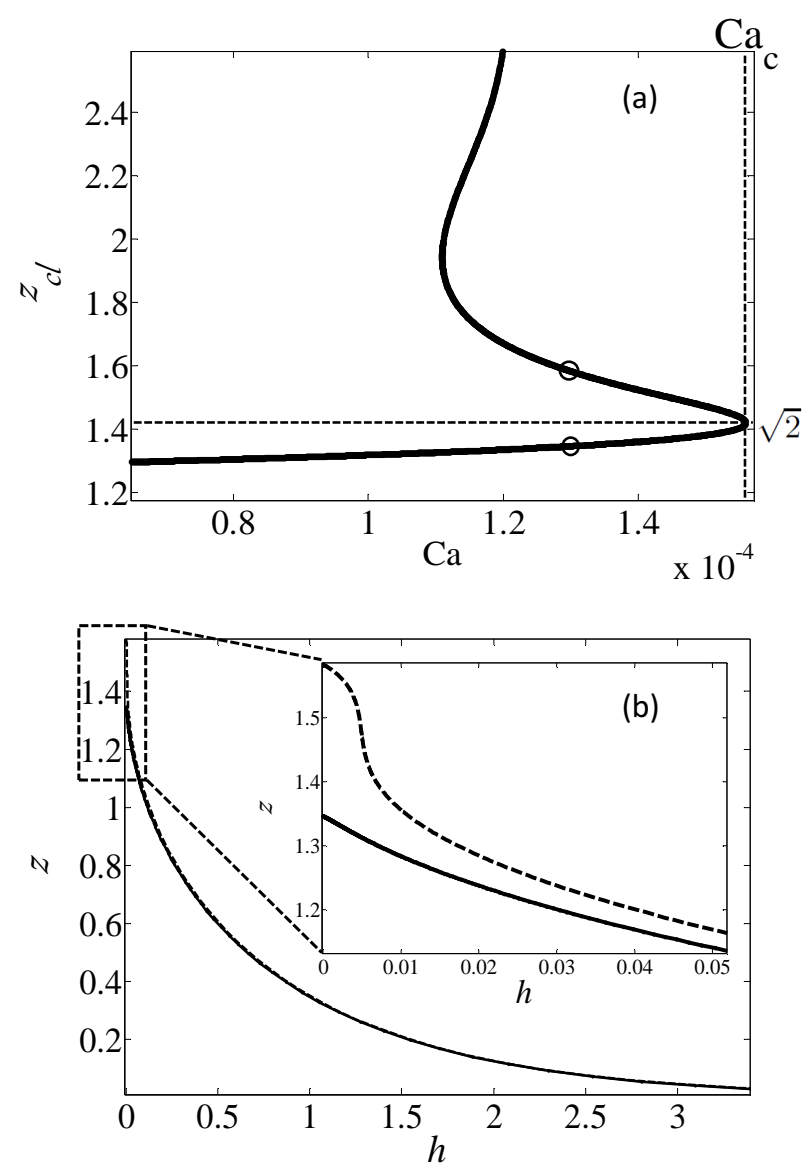

Figure 2.2: (a) Bifurcation diagram of stationary solutions, showing the meniscus rise $z_{\mathrm{cl}}$ (in units of $\ell_{c}$ ), as function of Ca for $\theta_{\mathrm{e}}=0.2$ and slip length $\lambda_{s}=10^{-5}$. Solid curve: result from a numerical solution of lubrication theory, see [13]. The horizontal dotted line indicates $z_{\mathrm{cl}}=\sqrt{2}$, which is the maximum rise height of a meniscus at equilibrium. (b) Interface profiles of the lower branch solution (solid curve) and the upper branch solution (dashed curve) for $\mathrm{Ca}=1.3 \times 10^{-4}$ (indicated by circles in (a)). The contact line position is at $z=z_{\mathrm{cl}}$, while $z=0$ at the bath. In the upper branch, the interface develops a finger close to the wall, making $z_{\mathrm{cl}}$ much larger than expected from the extrapolation of the far-field profile.

Below the horizontal dashed line of Fig. 2.2(a), the contact line position is a monotonically increasing function of speed. For very small $\mathrm{Ca}$, the capillary rise 
approaches its equilibrium value [30]

$$
z_{\mathrm{cl}}=\sqrt{2\left(1-\sin \theta_{\mathrm{e}}\right)} .
$$

As the speed increases, the lower branch solution ends at a maximum value $\mathrm{Ca}_{c}$ of the control parameter, which is the typical scenario for a saddle-node bifurcation [31]. At this point a transition toward a wetted state must occur, since all available steady states correspond to a smaller speed than the actual speed $\mathrm{Ca}_{c}$. The lower branch of the solution curve corresponds to a stable equilibrium, the upper branch to an unstable equilibrium [31]. Eventually, the contact line position goes to infinity, and the plate is covered completely by a film.

In $[3,11]$, the lower branch of the solution curve of Fig. 2.2(a) has been studied analytically, using the method of matched asymptotic expansions. This approach exploits the disparity of scales between the capillary length $\left(\approx 10^{-3} \mathrm{~m}\right)$, and the slip length $\left(10^{-9} \mathrm{~m}\right)$, relevant only very close to the contact line. The outer solution is controlled by a static balance between surface tension and gravity, enabling one to use the relationship (2.1) between capillary rise and contact angle, but with an "apparent" contact angle $\theta_{\mathrm{ap}}$ :

$$
z_{\mathrm{cl}}=\sqrt{2\left(1-\sin \theta_{\mathrm{ap}}\right)} .
$$

The maximum value of $z_{\mathrm{cl}}$ consistent with (2.2) is $z_{\mathrm{cl}}=\sqrt{2}$, which is realized for $\theta_{\mathrm{ap}}=0$. Since (2.2) does not allow for solutions above $z_{\mathrm{cl}}=\sqrt{2}$, a transition occurs as the apparent contact angle vanishes. The corresponding maximum capillary number $\mathrm{Ca}_{c}$ has been calculated in [11]. An inspection of Fig. 2.2a shows that the bifurcation indeed occurs very close to $z_{\mathrm{cl}}=\sqrt{2}$, and the value of $\mathrm{Ca}_{c}$ agrees quantitatively with the theoretical prediction, as well as with experiment [13, 14]. An experiment withdrawing a fiber also found the transition to occur at vanishing apparent contact angle [18].

In this Chapter, we will supply the missing description of the upper branch in terms of matched asymptotic expansions. To our knowledge, this is the first time both branches of a saddle-node bifurcation have been described using this method. Matching requires a new type of inner solution, as illustrated in Fig. 2.2(b). We show solutions on the upper and lower branches of the transition (circles in Fig. 2.2(a)), but corresponding to the same value of $\mathrm{Ca}$. It is clear from the main graph of Fig. 2.2(b) that the two solutions are virtually identical on the large scale, and thus correspond to the same value of the apparent contact angle $\theta_{\mathrm{ap}}$. However, the upper branch solution is distinguished by a different inner solution, which features a thin viscous "finger", only visible in the inset, showing a magnified region very close to the plate. As a result, the actual contact line position $z_{\mathrm{cl}}$ at the tip of the finger is significantly higher 
than $z_{\mathrm{cl}}$ obtained by extrapolating from the outer solution, and which gives $z_{\mathrm{cl}}$ on the lower branch.

This Chapter is organized as follows: we first recount the technique of matched asymptotic expansion used for finding the lower branch solutions, presented in [3, 11]. We then perform an asymptotic analysis for the upper branch solutions by considering another set of inner solutions, which displays the narrow finger shape as discussed above. We thus show that on the upper branch, the true contact line position is shifted upwards from its apparent value. We demonstrate that the upper and lower branch solutions thus found can be joined at the bifurcation point. In the final discussion, we relate the bifurcation theory approach to the result of matched asymptotic expansions, and discuss remaining unsolved problems.

\subsection{Lower branch and critical speed}

The lower branch of the bifurcation diagram was calculated in [11] using matched asymptotics, for the case that the angle at which the plate is withdrawn is small. In this section we present a brief summary of this calculation, and adapt it to the case of vertical withdrawal. In the matched asymptotic description, the solution is broken up into an inner and an outer region, and denoted by $h_{\text {in }}(x)$ and $h_{\text {out }}(x)$, respectively. The variable $x$ is defined relative to the contact line position: $z=z_{c l}-x$, see Fig. 2.1. The full solution is found by imposing appropriate matching conditions between the two solutions.

\subsubsection{Inner solution: lubrication approximation}

For simplicity, we restrict ourselves to the case of small equilibrium contact angles, $h_{\text {in }}^{\prime}(0)=\theta_{\mathrm{e}} \ll 1$, so we can use the lubrication approximation close to the contact line [9]. Since the length scale in the inner region is set by the slip length $\lambda_{s}$, which is much smaller than $\ell_{c}$, gravity is negligible. The corresponding lubrication equation reads [13]:

$$
h_{i n}^{\prime \prime \prime}=\frac{3 \mathrm{Ca}}{h_{i n}^{2}+3 \lambda_{s} h_{i n}} .
$$

Note that the presence of $\lambda_{s}$ makes for a much weaker singularity as $h_{i n}$ vanishes. For $\lambda_{s}=0$, no dynamical solution of (2.3) exists which makes a finite contact angle with the substrate. Thus a finite value of $\lambda_{s}>0$ is needed to allow the contact line to move relative to the substrate. We scale out $\lambda_{s}$ from the problem, by introducing the similarity solution

$$
h_{i n}(x)=3 \lambda_{s} H\left(\frac{x \theta_{\mathrm{e}}}{3 \lambda_{s}}\right), \quad \xi=\frac{x \theta_{\mathrm{e}}}{3 \lambda_{s}} .
$$


With these rescalings (2.3) can be expressed in terms of the dimensionless profile $H(\xi)$,

$$
H^{\prime \prime \prime}=\frac{\delta}{H^{2}+H},
$$

where we introduced a reduced capillary number $\delta=3 \mathrm{Ca} / \theta_{\mathrm{e}}^{3}$. The boundary conditions on the plate are

$$
H(0)=0 \quad \text { and } \quad H^{\prime}(0)=1 .
$$

Since (2.5) is a third order differential equation, one more condition is required. This condition will be obtained from the matching to the outer solution.

Away from the contact line, where $H \gg 1$, (2.5) further reduces to

$$
y^{\prime \prime \prime}=\frac{1}{y^{2}},
$$

where we have put $H(\xi)=\delta^{1 / 3} y(\xi)$. This equation has an exact solution, whose properties have been summarized in [32]. In parametric form, a solution with $y(0)=$ 0 reads

$$
\left.\begin{array}{l}
\xi=\frac{2^{1 / 3} \pi A i(s)}{\beta(\alpha A i(s)+\beta B i(s))} \\
y=\frac{1}{(\alpha A i(s)+\beta B i(s))^{2}}
\end{array}\right\} s \in\left[s_{1}, \infty[,\right.
$$

where $A i$ and $B i$ are the two Airy functions [33]. The limit $\xi \rightarrow 0$ corresponds to $s \rightarrow \infty$, the opposite limit $\xi \rightarrow \infty$ to $s \rightarrow s_{1}$, where $s_{1}$ is a root of the denominator of (2.8):

$$
\alpha A i\left(s_{1}\right)+\beta B i\left(s_{1}\right)=0 .
$$

Since the solution extends to $s=\infty, s_{1}$ has to be the largest root of (2.9).

The solution $y(\xi)$ is thus characterized by $\alpha, \beta$ and $s_{1}$, but only two of these parameters are independent due to (2.9). As detailed in [11], the constant $\beta$ can be determined by matching (2.8), which is valid only for $\xi \gtrsim 1$, to a solution of (2.5), which includes the effect of the cutoff and is thus valid down to the position $\xi=0$ of the contact line. It was found that

$$
\beta^{2}=\pi \exp (-1 /(3 \delta)) / 2^{2 / 3}+\mathscr{O}(\delta),
$$

which eliminates one of the remaining two free parameters. The last parameter $s_{1}$ will be eliminated below by matching the large scale asymptotics of $y(\xi)$ to the outer solution of the problem. To this end, we need the behavior of $y(\xi)$ for large $\xi$, which can be obtained from (2.8):

$$
y(\xi)=\frac{1}{2} \kappa_{y} \xi^{2}+b_{y} \xi+\mathscr{O}(1),
$$


where

$$
\kappa_{y}=\left(\frac{2^{1 / 6} \beta}{\pi A i\left(s_{1}\right)}\right)^{2}, \quad b_{y}=\frac{-2^{2 / 3} A i^{\prime}\left(s_{1}\right)}{A i\left(s_{1}\right)}
$$

\subsubsection{Outer solution: liquid reservoir}

The outer solution $h_{\text {out }}(x)$, valid away from the contact line, is governed by a balance between surface tension and gravity [30]:

$$
\kappa \equiv \frac{h_{\text {out }}^{\prime \prime}}{\left(1+h_{\text {out }}^{\prime 2}\right)^{3 / 2}}=z_{\mathrm{cl}}-x,
$$

where $\kappa$ is the curvature of the interface. Remember that $x=0$ at the contact line, and $x=z_{c l}$ at the height of the bath. The static balance (2.13) is to be solved subject to the boundary conditions

$$
h_{\text {out }}(0)=0, \quad h_{\text {out }}^{\prime}(0)=\theta_{\text {ap }} \quad \text { and } \quad h_{\text {out }}^{\prime}\left(z_{\mathrm{cl}}\right)=\infty,
$$

which contains the apparent contact angle $\theta_{\text {ap }}$ as sole parameter. A Taylor expansion of the outer solution leads to

$$
h_{\text {out }}(x)=\tan \theta_{\mathrm{ap}} x+\frac{1}{2} \kappa_{\mathrm{ap}} x^{2}+\mathscr{O}\left(x^{3}\right) .
$$

Integrating (2.13) once with respect to $x$, we obtain

$$
1-\frac{h_{\mathrm{out}}^{\prime}}{\left(1+h_{\mathrm{out}}^{\prime 2}\right)^{1 / 2}}=\frac{1}{2}\left(z_{\mathrm{cl}}-x\right)^{2},
$$

where the boundary condition $h_{\text {out }}^{\prime} \rightarrow \infty$ at the position of the reservoir $\left(x=z_{\mathrm{cl}}\right)$ is used. Evaluating (2.16) at the contact line position $(x=0)$ and using the geometrical connection $\sin \theta=h_{\text {out }}^{\prime} / \sqrt{1+h_{\text {out }}^{\prime 2}}$ as well as (2.13), we find

$$
\kappa_{a p}=z_{\mathrm{cl}}=\sqrt{2\left(1-\sin \theta_{a p}\right)}
$$

as quoted in the introduction. Our main interest in this Chapter is the neighborhood of the bifurcation, i.e. the region of small $\theta_{a p}$. Thus for the sake of simplicity we contend ourselves with the leading-order expressions for small $\theta_{a p}$ and find:

$$
h_{\text {out }}(x)=\theta_{\mathrm{ap}} x+\frac{1-\theta_{a p} / 2}{\sqrt{2}} x^{2}+\mathscr{O}\left(x^{3}\right) .
$$




\subsubsection{Matching: lower branch}

To match the two solutions on the lower branch we first write the inner solution in term of the original variables,

$$
h_{\text {in }}(x)=\delta^{1 / 3}\left[\frac{\kappa_{y} \theta_{\mathrm{e}}^{2} x^{2}}{6 \lambda_{s}}+b_{y} \theta_{\mathrm{e}} x+\mathscr{O}(1)\right] .
$$

Comparing this to (2.17), we find the matching conditions

$$
\begin{aligned}
\theta_{\mathrm{ap}} & =\delta^{1 / 3} b_{y} \theta_{\mathrm{e}} \\
2-\theta_{\mathrm{ap}} & =\sqrt{2} \delta^{1 / 3} \frac{\kappa_{y} \theta_{\mathrm{e}}^{2}}{3 \lambda_{s}} .
\end{aligned}
$$

Adding these two conditions leads to an equation for $s_{1}$ as a function of $\delta$ :

$$
\frac{2}{\theta_{\mathrm{e}} \delta^{1 / 3}}+\frac{2^{2 / 3} A i^{\prime}\left(s_{1}\right)}{A i\left(s_{1}\right)}=\frac{2^{1 / 6} \exp [-1 /(3 \delta)]}{3 \pi A i^{2}\left(s_{1}\right) \lambda_{s} / \theta_{\mathrm{e}}} .
$$

Once $s_{1}$ is known, one can compute the apparent contact angle

$$
\frac{\theta_{\mathrm{ap}}}{\theta_{\mathrm{e}}}=-\frac{2^{2 / 3} \delta^{1 / 3} A i^{\prime}\left(s_{1}\right)}{A i\left(s_{1}\right)} .
$$

Analysis of (2.21) shows [11] that solutions cease to exist above a critical reduced speed $\delta_{c}$, for which the apparent contact angle goes to zero. According to (2.22), this occurs when the Airy function takes its global maximum, $A i^{\prime}\left(s_{1}\right)=0$, corresponding to $s_{\max }=-1.088 \cdots$ (cf. Fig. 2.3). This gives a critical speed

$$
\delta_{c}=\frac{1}{3}\left[\ln \left(\frac{\delta_{c}^{1 / 3} \theta_{\mathrm{e}}^{2}}{2^{5 / 6} 3 \pi\left(A i\left(s_{\max }\right)\right)^{2} \lambda_{s}}\right)\right]^{-1} ;
$$

remember that $\delta_{c}$ is related to the critical capillary number by $\mathrm{Ca}_{\mathrm{c}}=\delta_{c} \theta_{\mathrm{e}}^{3} / 3$. This completes the description of the lower branch of Fig. 2.2a, up to the critical capillary number. However, the analysis so far gives no clue as to what happens beyond the transition, and thus does not explain the nature of the transition.

\subsection{Upper branch and bifurcation diagram}

We now turn to the upper branch of the bifurcation diagram close to the critical speed. Clearly, this branch cannot be described in terms of (2.2), since $z_{\mathrm{cl}}$ rises above the 


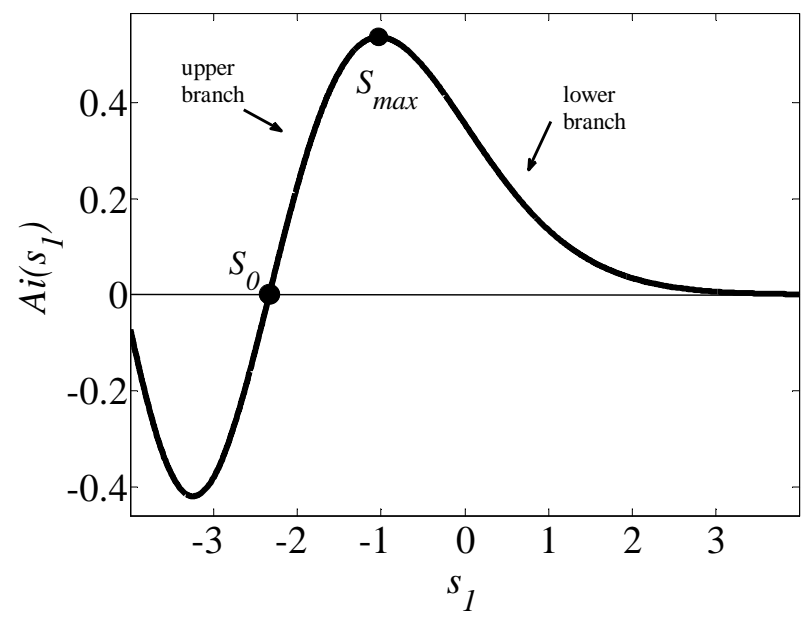

Figure 2.3: Airy function $A i\left(s_{1}\right)$. The critical point occurs at $s_{\max }=-1.088 \cdots$ where the Airy function takes its maximum value. The lower and upper branches of the bifurcation diagram correspond to $s_{1} \geq s_{\max }$ and $s_{0}<s_{1}<s_{\max }$ respectively, where $s_{0}=-2.338 \cdots$ is the rightmost zero of $A i\left(s_{1}\right)$.

maximum value of $\sqrt{2}$ corresponding to $\theta_{\mathrm{ap}}=0$. The critical point is attained when $A i\left(s_{1}\right)$ takes its maximum value, at $s_{1}=s_{\max }=-1.088 \cdots$ (cf. Fig. 2.3). The rest of the lower branch corresponds to values $s_{1}>s_{\max }$. This suggests that the upper branch can be described in terms of solutions on the other side of the maximum, $s_{1}<s_{\max }$, and below we will work out this idea.

\subsubsection{Matching: upper branch}

An inspection of (2.12) shows that $b_{y}$ is strictly negative in the domain $s_{0}<s_{1}<s_{\max }$, where $s_{0}=-2.338 \cdots$ is the rightmost zero of the Airy function (cf. Fig. 2.3). Thus we cannot impose the same matching as in the previous section, since (2.19) would imply a negative apparent contact angle. We get around this problem by noting that the large scale asymptotics of the inner solution (2.11) is a parabola that has two zeros, $\xi=0$ and $-2 b_{y} / \kappa_{y}$. The former coincides with the actual position of the contact line, while we interpret the latter as the 'apparent' position of the contact line

$$
\xi_{\mathrm{ap}}=\frac{-2 b_{y}}{\kappa_{y}} .
$$




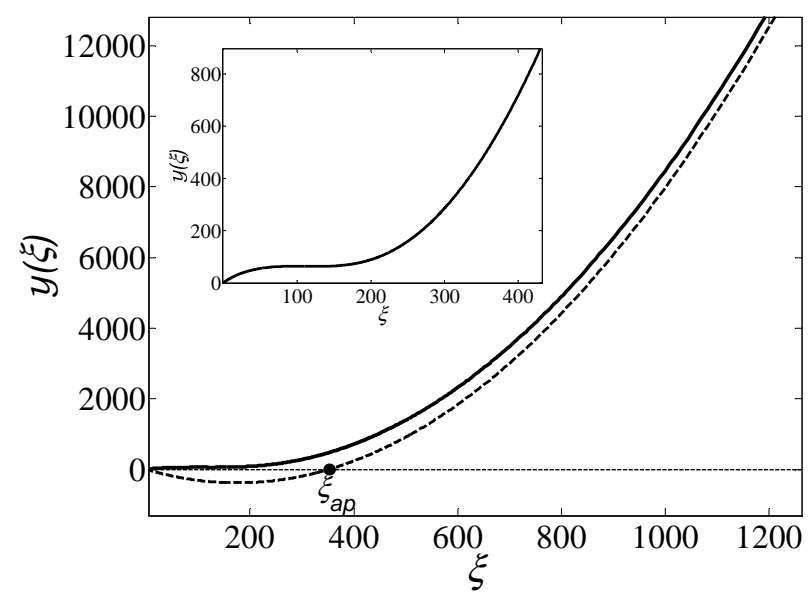

Figure 2.4: The profile (2.8) for $s_{1}=-2.0$ and $\delta=0.063$ (solid line). The dashed line is the asymptotic form (2.11) of the profile for large $\xi$; from a large scale the contact line appears to be located at $\xi_{\text {ap }} \sim 350$. The inset is a zoom on the contact line region, showing a narrow finger which ends at the contact line $\xi=0$.

Figure 2.4 illustrates how a typical solution (2.8) (solid line) approaches its asymptotic limit (2.11) (dashed line), so that the relative distance between the two curves goes to zero for large $\xi$. Matching the next (constant) term in (2.11) would be achieved only at the next order of the asymptotic expansion. Extrapolating the largescale solution to the plate position, the contact line appears to be located at $\xi=\xi_{a p}$, while its actual position is at $\xi=0$. This is due to the narrow finger exhibited by solutions $y(\xi)$ for the parameter range $s_{0}<s_{1}<s_{\max }$ (cf. inset of Fig. 2.4). This finger was already visible in the numerical solutions shown in Fig. 2.2(b).

Expanding (2.11) relative to the apparent contact line position $\xi_{\mathrm{ap}}$, we obtain

$$
\begin{aligned}
y(\xi) & =\frac{1}{2} \kappa_{y} \xi^{2}+b_{y} \xi+\mathscr{O}(1) \\
& =\frac{1}{2} \kappa_{y}\left(\xi-\xi_{\text {ap }}\right)^{2}-b_{y}\left(\xi-\xi_{\text {ap }}\right)+\mathscr{O}(1) .
\end{aligned}
$$

The prefactor of the linear term, $-b_{y}$, is now strictly positive on $s_{0}<s_{1}<s_{\max }$ and can therefore be matched to a 'shifted' bath solution:

$$
\begin{aligned}
h_{\text {out }}(x) & =\theta_{\mathrm{ap}}\left(x-x_{\mathrm{ap}}\right)+\frac{1-\theta_{a p} / 2}{\sqrt{2}}\left(x-x_{\mathrm{ap}}\right)^{2} \\
& +\mathscr{O}\left(\left(x-x_{\mathrm{ap}}\right)^{3}\right) .
\end{aligned}
$$




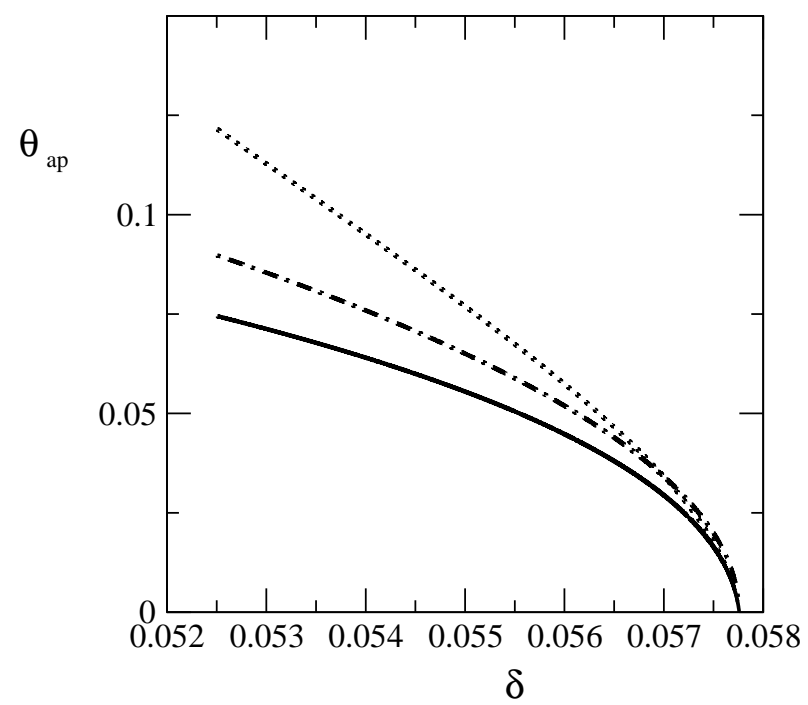

Figure 2.5: The apparent contact angle $\theta_{a p}$ as function of $\delta$ for lower branch (solid line) and upper branch (dotted line). The two curves meet at $\delta=\delta_{c} \approx 0.0578$, where $\theta_{a p}=0$. The dot-dashed line corresponds to (2.37) below.

Retracing the steps of the previous section, one finds the equation for $s_{1}$ as

$$
\frac{2}{\theta_{\mathrm{e}} \delta^{1 / 3}}-\frac{2^{2 / 3} A i^{\prime}\left(s_{1}\right)}{A i\left(s_{1}\right)}=\frac{2^{1 / 6} \exp [-1 /(3 \delta)]}{3 \pi A i^{2}\left(s_{1}\right) \lambda_{s} / \theta_{\mathrm{e}}} .
$$

This differs from (2.21) only by a minus sign in the second term of the left hand side. Similarly, the apparent contact angle follows as

$$
\frac{\theta_{\mathrm{ap}}}{\theta_{\mathrm{e}}}=\frac{2^{2 / 3} \delta^{1 / 3} A i^{\prime}\left(s_{1}\right)}{A i\left(s_{1}\right)}
$$

with a change in sign. In Fig. 2.5, we plot $\theta_{a p}$ as function of $\delta$ for both lower branch (solid line) and upper branch (dotted line). Both curves meet at $\delta=\delta_{c}$, where the apparent contact angle vanishes. Thus the apparent contact angle decreases as the transition is approached from below, and then increases again on the upper branch.

\subsubsection{Meniscus rise}

Let us now express the new solution in terms of the meniscus rise $z_{\mathrm{cl}}$ and compare to the bifurcation diagram of Fig. 2.2(a). To do so, we need to take into account the 
difference between the real position of the contact line at $\xi=0$, and the apparent position $\xi_{\text {ap }}$. This difference comes on top of the meniscus rise of the outer solution, given by (2.2), so that in original variables

$$
z_{\mathrm{cl}}=\sqrt{2}\left(1-\theta_{\mathrm{ap}} / 2\right)+\xi_{\mathrm{ap}} \frac{3 \lambda_{s}}{\theta_{\mathrm{e}}},
$$

once more expanding for small $\theta_{\mathrm{ap}}$. Rewriting (2.25) in terms of the outer variables $x$ and $h$, and comparing it to (2.26), we obtain

$$
b_{y}=\frac{-\theta_{a p}}{\delta^{1 / 3}}, \quad \kappa_{y}=\frac{3 \cdot \sqrt{2} \lambda_{s}}{\delta^{1 / 3} \theta_{e}} .
$$

Now using (2.24), one finds

$$
\xi_{\mathrm{ap}} \frac{3 \lambda_{s}}{\theta_{\mathrm{e}}}=\sqrt{2} \theta_{\mathrm{ap}},
$$

giving the contact line position directly in terms of $\theta_{\text {ap }}$. Thus we finally arrive at:

$$
z_{\mathrm{cl}}=\sqrt{2} \begin{cases}1-\theta_{\mathrm{ap}} / 2 & \text { for } z_{\mathrm{cl}} \leq \sqrt{2} \\ 1+\theta_{\mathrm{ap}} / 2 & \text { for } z_{\mathrm{cl}} \geq \sqrt{2}\end{cases}
$$

To test (2.31), in Fig. 2.6 we replot $z_{\mathrm{cl}}$ as function of $\delta$ for the same parameter values as those of Fig. 2.2(a). The solid curve is the numerical solution of the improved lubrication theory, the dashed lines represent (2.31), with $\theta_{a p}$ calculated for the lower and upper branches, respectively. The agreement of the numerical result and the analytical results is very good for both branches.

\subsubsection{Comparison to bifurcation theory}

Returning to the bifurcation argument presented in the introduction, the transition occurs because the solution curve folds over, and is thus guaranteed to have a local expansion of the form

$$
\delta-\delta_{c}=a_{1}\left(z_{\mathrm{cl}}-\sqrt{2}\right)^{2}+\mathscr{O}\left(z_{\mathrm{cl}}-\sqrt{2}\right)^{3} .
$$

To discover the local behavior resulting from the matched asymptotics, we insert the expansion

$$
\delta-\delta_{c}=\delta_{1} \delta s_{1}+\delta_{2} \delta s_{1}^{2}, \quad \delta s_{1}=s_{1}-s_{\max }
$$

into (2.21) and (2.27), respectively. On the basis of (2.32), we would expect $\delta_{1}$ to vanish and $\delta_{2}$ to be the same above and below the transition. 


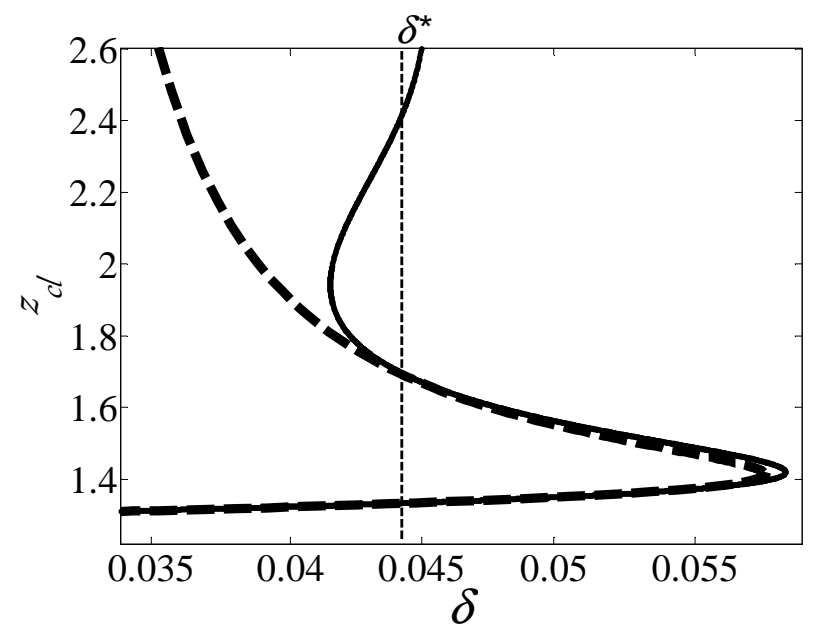

Figure 2.6: Meniscus rise $z_{\mathrm{cl}}$ as function of $\delta$ for $\theta_{\mathrm{e}}=0.2$ and the slip length $\lambda_{s}=$ $10^{-5}$. Solid line: Same as in Fig. 2.2a. Dashed lines are (2.31), with $\theta_{a p}$ from (2.21), (2.22) (lower branch) and (2.27),(2.28) (upper branch). For large $z_{c l}$, the curve approaches a limiting speed $\delta^{*}$.

Instead, we find

$$
\delta_{1}=-\frac{3 \sigma 2^{2 / 3} s_{\max } \theta_{\mathrm{e}} \delta_{c}^{7 / 3}}{2\left(\delta_{c}+1\right)},
$$

where $\sigma= \pm 1$ above and below the transition, respectively; the coefficient $\delta_{2}$ does not have a definite sign. Clearly, the bifurcation curve as predicted by matched asymptotics is not smooth at the bifurcation point. Indeed, close inspection of Fig. 2.6 reveals that the dashed line does not have a vertical tangent at the turning bifurcation point [11]. Instead, the "correct" behavior emerges only in the limit of small slip length $\lambda_{s} \rightarrow 0$ or $\delta_{c} \rightarrow 0$. In this limit, we find

$$
\delta_{2}=3 s_{\max } \delta_{c}^{2}
$$

which is indeed the same above and below the transition. Thus the first term of (2.33) is negligible (and the structure (2.32) is valid) for $\delta s_{1} \gg \delta_{c}^{1 / 3}$.

In addition, from (2.22) and (2.28) we find that to leading order

$$
\theta_{a p}=-2^{2 / 3} \theta_{\mathrm{e}} s_{\max } \delta_{c}\left|\delta s_{1}\right|,
$$

which is valid both above $\left(\delta s_{1}<0\right)$ and below $\left(\delta s_{1}>0\right)$ the transition. Thus in 
summary we find

$$
\theta_{a p}=2^{2 / 3} \theta_{\mathrm{e}}\left|\frac{s_{\max }}{3}\right|^{1 / 2} \delta_{c}^{-2 / 3}\left|\delta-\delta_{c}\right|^{1 / 2}
$$

which is valid for $\theta_{a p} \gg \delta_{c}^{2 / 3}$. The asymptotic local behavior (2.37) is plotted in Fig. 2.5 as the dot-dashed line. Its behavior for small $\theta_{a p}$ is hard to distinguish from (2.22) and (2.28).

\subsection{Discussion}

Matched asymptotics clearly gives a quantitative description of both branches of the saddle-node bifurcation. In the case of the lower branch, this holds true for the entire branch down to vanishing speed. The behavior of the upper branch, on the other hand, is considerably more complicated, as described in $[14,15]$. Following the upper branch, one encounters an infinite sequence of saddle-node bifurcations, as the solution curve oscillates around a second characteristic speed $\delta^{*}$. The oscillations of the solution curve, which are due to oscillations of the interface profile [16], are damped very quickly, so at a capillary rise of a few times the capillary length the solution effectively corresponds to a film of constant thickness covering the plate. This film, of thickness $h^{*}=\sqrt{\delta^{*} \theta_{\mathrm{e}}^{3}}$, is maintained by a balance of viscosity and gravity. Experimentally, this film has indeed been realized when a plate is withdrawn with speed above $\delta^{*}[15]$.

Our present analysis is not able to capture this feature, since gravity is not included in the balance (2.3), which describes the inner solution. As the finger seen in the inset of Fig. 2.4 grows in length, the hydrostatic pressure difference across it becomes significant, and the solution fails. An estimate of the capillary rise at which this occurs will be given by the point where the theoretical curve crosses the vertical line $\delta^{*}$, where gravity and viscosity balances. Using (2.31) and (2.37), this leads to the estimate

$$
z_{\mathrm{cl}}-\sqrt{2} \approx \theta_{\mathrm{e}} \delta_{c}^{-2 / 3} \sqrt{\delta_{c}-\delta^{*}}
$$

for the rise at which gravity becomes significant. Using $\delta_{c} \approx 0.058$ and $\delta^{*} \approx 0.044$, this leads to $z_{\mathrm{cl}}-\sqrt{2} \approx 0.16$, which agrees reasonably well with Fig. 2.6.

It is worth reviewing the relative merits of bifurcation theory and those of matched asymptotics, which are complementary. Within bifurcation theory, once one understands the origin of the transition as a fold of the solution curve of Fig. 2.2(a), the local structure or order of the transition results automatically. In addition, it is clear that the transition must correspond to moving from a stable branch to an unstable 
branch [31]. As always, the disadvantage is that the critical parameters of the transition, such as $\delta_{c}$, cannot be calculated within bifurcation theory.

Matched asymptotics, on the other hand, requires a detailed calculation, which reveals the full spatial structure of the solution above and below the transition, as well as the values of all the critical parameters, calculated directly from the system parameters. For example, it is shown that the upper branch solution contains an additional, and unforeseen structure, which is the finger seen in Fig. 2.2(b). However, the local structure (2.32) of the saddle-node bifurcation emerges from the calculation only in the limit $\lambda_{s} \rightarrow 0$ or $\delta_{c} \rightarrow 0$. Note the subtle point that the two branches as predicted by asymptotics do not fit together to form a differentiable curve. The reason is that both parts present very different spatial features, and hence agreement between matched asymptotic expansion and bifurcation theory is achieved in a pointwise fashion only. It would be an interesting project to see if the next order of the asymptotic expansion will reproduce the next term in the expansion about the bifurcation point.

The stability properties of the two branches have not yet been investigated within matched asymptotics, and remain a non-trivial problem: the task is to properly separate the timescales of the inner and outer solutions. The result is expected to be an effective dynamics [13], in which the solution moves quasistatically along the solution curve. This picture of quasi-steady dynamics was confirmed in experiments where the plate velocity was taken above the critical speed [14]. It was found that during the deposition of the liquid film, the upward motion of the contact line follows the bifurcation curve perfectly when replacing the plate velocity by the relative velocity of the contact line, i.e. $U-d z_{c l} / d t$.

In conclusion, we have calculated the upper and lower branches of a saddlenode bifurcation using matched asymptotics. We are not aware of any other example of this having been done before. It would be of great interest to develop a more general framework of correspondences between certain types of bifurcations, and the matched asymptotics needed to describe them. 


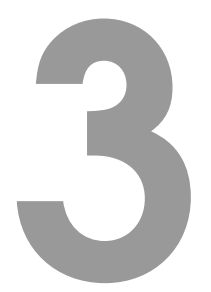

\section{Maximum speed of dewetting on a fiber *}

A solid object can be coated by a nonwetting liquid since a receding contact line cannot exceed a critical speed. We theoretically investigate this forced wetting transition for axisymmetric menisci on fibers of varying radii. First, we use a matched asymptotic expansion and derive the maximum speed of dewetting. For all radii we find the maximum speed occurs at vanishing apparent contact angle. To further investigate the transition we numerically determine the bifurcation diagram for steady menisci. It is found that the meniscus profiles on thick fibers are smooth, even when there is a film deposited between the bath and the contact line, while profiles on thin fibers exhibit strong oscillations. We discuss how this could lead to different experimental scenarios of film deposition.

\subsection{Introduction}

A convenient way to deposit a thin liquid layer on a surface is by withdrawing a solid from a liquid reservoir. The film is dragged along with the solid due to the viscous friction of the liquid. This principle is known as dip-coating and is a commonly used technique in industrial contexts $[25,26]$. Once deposited on the surface, the film often has a thickness as predicted by Landau, Levich [17] and Derjaguin [34], scaling with

${ }^{*}$ Published as: T.S. Chan, T. Gueudré and J.H. Snoeijer, Maximum speed of dewetting on a fiber, Phys. Fluids 23, 112103 (2011). 
speed $U$ of withdrawal as $h \propto U^{2 / 3}$. Recently, however, a different class of solutions were identified, which are much thicker and scale as $h \propto U^{1 / 2}$ [15]. These thick films were indeed realized experimentally in the case where the solid was partially wetting.

The conditions of partial wetting introduces another interesting feature, namely that the film entrainment only appears above a critical velocity of withdrawal $[9,21$, $27,35,36]$. Below this speed the contact line finds at a steady position, indicated as the meniscus rise $\Delta$ (Fig. 3.1). Due to viscous drag between the liquid and the solid, the dynamical position of $\Delta$ is higher than at equilibrium. This means that the apparent contact angle $\theta_{a p}$ of the dynamical meniscus is smaller than the equilibrium angle $\theta_{e}$. The simplest interpretation of the transition to film deposition is that the apparent contact angle $\theta_{a p} \rightarrow 0$ at a critical plate velocity. This idea was already postulated by Derjaguin and Levi [24], although the energy argument given by de Gennes [35] suggested a nonzero $\theta_{a p}$ at the transition. The hypothesis of $\theta_{a p}=0$, however, was given a rigorous mathematical basis (for a flat solid) by asymptotic expansions of the lubrication equations [3,11]. Actually, it was shown by [9] that de Gennes energy argument can be extended to incorporate interface curvature: this exactly gives the lubrication equation, meaning that also the energy argument leads to a zero $\theta_{a p}$ at the transition. This theory gives a simple prediction for the maximum rise, based on the static meniscus solution with vanishing contact angle - for a fiber of radius $r_{0}$ this simply becomes [30,37]

$$
\Delta_{\max } \simeq \begin{cases}r_{0}\left(\ln \frac{4 \ell_{c}}{r_{0}}-c\right) & \text { for } r_{0} \ll \ell_{c} \\ \sqrt{2} \ell_{c} & \text { for } r_{0} \gg \ell_{c} .\end{cases}
$$

Here $\ell_{c}=(\gamma / \rho g)^{1 / 2}$ is the capillary length based on surface tension $\gamma$, density $\rho$, gravity $g$ and $c$ is Euler's constant $(0.57721)$. At intermediate radii $r_{0} \sim \ell_{c}$, the maximum rise can be determined numerically.

Experimentally, the description of the forced wetting transition has remained ambiguous. The condition of a vanishing apparent contact angle was convincingly shown by Sedev \& Petrov [18]. When withdrawing fibers or thin cylinders $\left(r_{0} / \ell_{c} \sim\right.$ $0.06-1)$, they found a maximum rise of the meniscus consistent with (3.1). Using cylinders of larger radii $\left(r_{0} / \ell_{c} \sim 10\right)$, Maleki et al. [38] found zero or nonzero $\theta_{a p}$ at the transition, depending on the way $\theta_{a p}$ was determined. When using the criterion based on the meniscus height, the transition was found slightly before reaching $\Delta_{\max }$. Yet another set of experiments using a flat plate $\left(r_{0} / \ell_{c}=\infty\right)$ displayed a transition to film deposition clearly before reaching the maximum rise [12, 14]. Still, during the unsteady entrainment phase the maximum recorded speed was reached exactly at $\sqrt{2} \ell_{c}$. Note that in these experiments, the deposited liquid was not simply 


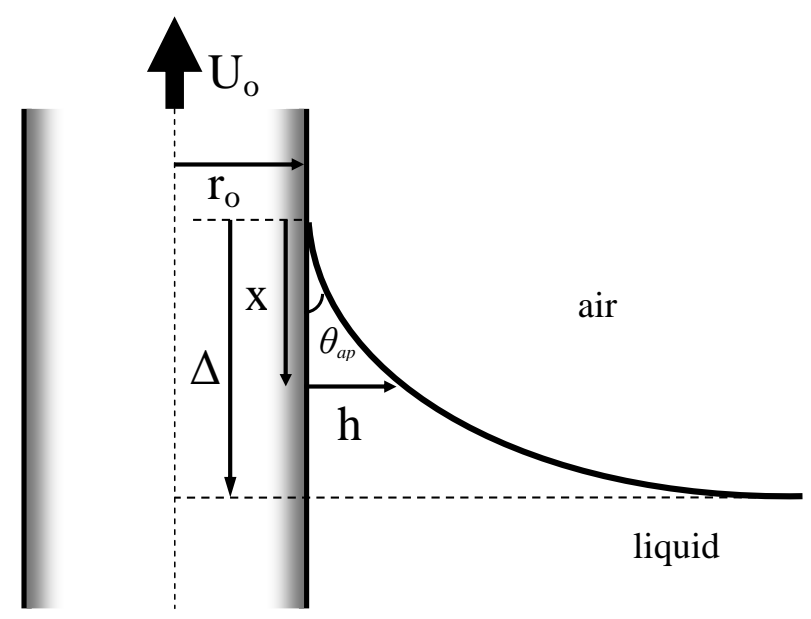

Figure 3.1: Schematic representation of the dip-coating setup: A fiber or cylinder of radius $r_{0}$ is withdrawn with speed $U_{0}$ from a bath of viscous liquid. The axisymmetric meniscus profile is characterized by $h(x)$, while $\Delta$ denotes the maximum rise above the reservoir.

the Landau-Levich-Derjaguin film, but gave rise to thick films and even shock solutions. It was argued that the presence of these dynamical solutions are related to the pre-critical onset of entrainment [13], but an explanation is still lacking.

An additional complexity is that the contact line can spontaneously develop sharp corner structures, or even zig-zags. This has been observed in dip-coating [2], splashing [39], immersion lithography [4, 40] and for drops sliding down an inclined plane [1, 41]. The conical structure of the interface near the contact line renders the problem truly three-dimensional, which affects the balance of the capillary forces [42]. For sliding drops, it has been observed experimentally and described by a 3D lubrication model, that this change in geometry indeed leads to a nonzero apparent contact angle at the transition to liquid deposition [43, 44]. This raises the question of how the geometry of the flow can influence the critical speed of wetting [16].

In this Chapter, we theoretically study the withdrawal of fibers of arbitrary radii. By varying the ratio $r_{0} / \ell_{c}$, we continuously cover the range from thin fibers to the flat plate. First, we extend the asymptotic analysis that was previously done for the flat plate $[3,11]$ (see also Chapter 2) to the limit of thin fibers (Sec. 3.2). To resolve the singularity of viscous stress near the contact line $[6,45]$, we introduce a slip length $\lambda_{s}[46,47]$. Other types of microscopic regularization will give similar results [9]. Typical values for the slip and capillary lengths are $\lambda_{s} \sim 10^{-9} \mathrm{~m}$ and $\ell_{c} \sim 10^{-3} \mathrm{~m}$ 
respectively. We can thus exploit the hierarchy of length scales

$$
\lambda_{s} \ll r_{0} \ll \ell_{c},
$$

and perform a matched asymptotic expansion. The control parameter is the capillary number $\mathrm{Ca}=U_{0} \eta / \gamma$, which is the speed of withdrawal scaled by viscosity $\eta$ and surface tension $\gamma$. The analysis yields the critical capillary number, which depends on the value of $r_{0}$, and confirms that the maximum speed coincides with $\theta_{a p}=0$, for all fiber radii $r_{0}$. In this sense, the change in geometry does not qualitatively change the nature of the critical point. However, striking differences do show up when computing numerically the complete bifurcation diagrams for all steady solutions (Sec. 3.3). These diagrams include solution branches above $\Delta_{\max }$ that are unstable, but which have been observed as transients during film deposition for the plate case [14]. We find that for small fiber radii much below $\ell_{c}$, the steady solutions no longer smoothly join the film solutions that mediate the deposition. In the Discussion section we speculate that this is why, experimentally, it is easier to approach the critical point for thin fibers (Sec. 3.4).

\subsection{Asymptotic analysis}

We compute the shape of an axisymmetric meniscus on a fiber of radius $r_{0}$ using the method of matched asymptotic expansions. The interface is characterized by $h(x)$, as sketched in Fig. 3.1. The matching procedure is outlined schematically in Fig. 3.2. At small scales, the dominant balance is between viscosity $\eta$ and surface tension $\gamma$, and is characterized by the capillary number $\mathrm{Ca}$. Viscous effects can be neglected on large scales, for which the interface profile is that of a static meniscus. The problem is closed by matching the inner and outer solutions. The analysis provides the meniscus rise $\Delta$ as a function of $\mathrm{Ca}$ as well as the critical speed, both of which can be observed experimentally. We consider both large fiber radii $\left(r_{0} \gg \ell_{c}\right)$ and small fiber radii $\left(r_{0} \ll \ell_{c}\right)$. In all cases we take $r_{0}$ and $\ell_{c}$ to be macroscopic and much greater than the microscopic cutoff. Throughout the analysis, we scale all lengths by the capillary length, i.e. $\ell_{c}=1$.

\subsubsection{Inner solution: lubrication approximation}

To distinguish the solution $h$ in the inner region and the outer region, we denote $h_{\text {in }}(x)$ as the solution in inner region and $h_{\text {out }}(x)$ as the solution in outer region. The characteristic length scale for the inner solution comes from the cutoff of the viscous singularity, which here we take the slip length $\lambda_{s}$. Since typical interface curvatures 


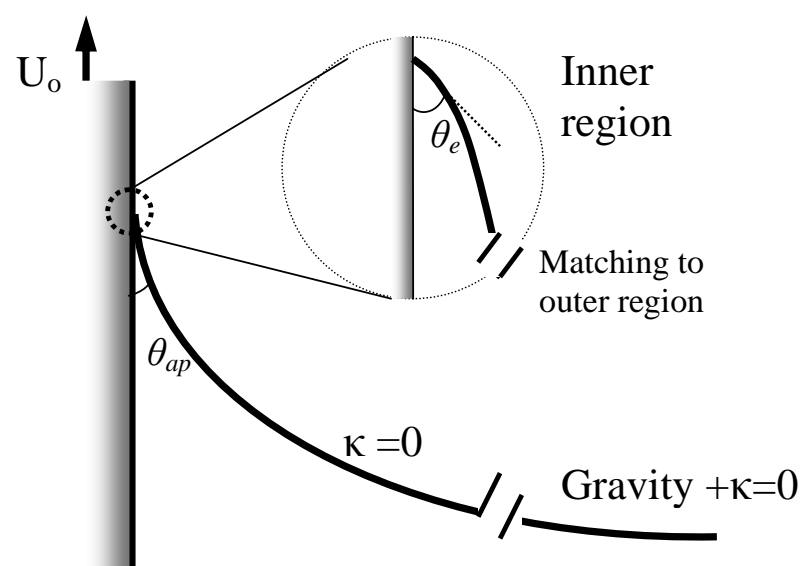

Outer region

Figure 3.2: Schematic diagram showing the different asymptotic regions for the case of a thin fiber. The inner region originates from a balance between viscosity and surface tension. It has a microscopic contact angle $\theta_{e}$. The outer region is a static meniscus joining a fiber with an apparent contact angle $\theta_{a p}$. When the fiber radius $r_{0} \ll \ell_{c}$, the outer profile is further separated into two regions [37].

turn out $\sim \mathrm{Ca}^{1 / 3} / \lambda_{s}$, as can be observed from the rescalings below, we can neglect the curvature contribution due to axisymmetry, which is of order $1 / r_{0}$. Hence, for the inner solution we can follow the analysis by Eggers [3,11], which was originally derived for the flat plate, and discussed in Chapter 2 as well. For completeness, we briefly summarize the analysis and the central results.

By restricting the analysis to small contact angles, $h_{i n}^{\prime}(0)=\theta_{e} \ll 1$, one can determine $h(x)$ from the lubrication approximation [10]:

$$
h_{i n}^{\prime \prime \prime}=\frac{3 \mathrm{Ca}}{h_{i n}^{2}+3 \lambda_{s} h_{i n}} .
$$

Since the slip length $\lambda_{s}$ is the only length scale, we rescale the solutions according to

$$
h_{i n}(x)=3 \lambda_{s} H\left(\frac{x \theta_{e}}{3 \lambda_{s}}\right), \quad \xi=\frac{x \theta_{e}}{3 \lambda_{s}} .
$$

Hence (3.4) reduces to 


$$
H^{\prime \prime \prime}=\frac{\delta}{H^{2}+H},
$$

where we introduced a reduced capillary number $\delta=3 \mathrm{Ca} / \theta_{e}^{3}$. The boundary conditions are

$$
\begin{aligned}
& H(\xi=0)=0, \\
& H^{\prime}(\xi=0)=1
\end{aligned}
$$

and the asymptotic behavior that has to be matched to the outer solution. Away from the contact line, where $H \gg 1$, (3.5) further reduces to

$$
y^{\prime \prime \prime}=\frac{1}{y^{2}},
$$

where we have put $H(\xi)=\delta^{1 / 3} y(\xi)$. This equation has an exact solution, whose properties have been summarized in [32]. In parametric form, a solution with $y(0)=$ 0 reads

$$
\left.\begin{array}{l}
\xi=\frac{2^{1 / 3} \pi A i(s)}{\beta(\alpha A i(s)+\beta B i(s))} \\
y=\frac{1}{(\alpha A i(s)+\beta B i(s))^{2}}
\end{array}\right\} s \in\left[s_{1}, \infty[,\right.
$$

where $A i$ and $B i$ are Airy functions [33]. The limit $\xi \rightarrow 0$ corresponds to $s \rightarrow \infty$, the opposite limit $\xi \rightarrow \infty$ to $s \rightarrow s_{1}$, where $s_{1}$ is a root of the denominator of (3.9):

$$
\alpha A i\left(s_{1}\right)+\beta B i\left(s_{1}\right)=0 .
$$

Since the solution extends to $s=\infty, s_{1}$ has to be the largest root of (3.10).

The solution $y(\xi)$ is thus characterized by three parameters $\alpha, \beta$ and $s_{1}$. Note that these are related according to (3.10), so that only two parameters are independent. The constant $\beta$ can be determined by matching (3.9), which is valid only for $\xi \gtrsim 1$, to a solution of (3.5), which includes the effect of the cutoff and is thus valid down to the position $\xi=0$ of the contact line [11]. It was found that

$$
\beta^{2}=\pi \exp (-1 /(3 \delta)) / 2^{2 / 3}+O(\delta),
$$

which eliminates one of the two free parameters. The remaining parameter will be eliminated below by matching the large scale asymptotics of $y(\xi)$ the outer solution 
of the problem. For that, we only need the asymptotic behavior of $y(\xi)$ for large $\xi$, which reads:

$$
y(\xi)=\frac{1}{2} \kappa_{y} \xi^{2}+b_{y} \xi+\mathscr{O}(1)
$$

where

$$
\kappa_{y}=\left(\frac{2^{1 / 6} \beta}{\pi A i\left(s_{1}\right)}\right)^{2}, \quad b_{y}=\frac{-2^{2 / 3} A i^{\prime}\left(s_{1}\right)}{A i\left(s_{1}\right)}
$$

\subsubsection{Outer solution: static meniscus}

At the scale of outer solution one can neglect viscous effects, and the profile is governed by surface tension and gravity. Thus equating the hydrostatic pressure and the capillary pressure gives

$$
\kappa=\Delta-x,
$$

where $\kappa$ is the curvature of the interface. Remind that we expressed all lengths in the capillary length $\ell_{c}=1$. The curvature can be expressed from the geometric relation

$$
\kappa=\frac{h_{\text {out }}^{\prime \prime}}{\left(1+h_{\text {out }}^{\prime 2}\right)^{3 / 2}}-\frac{1}{\left(r_{0}+h_{\text {out }}\right)\left(1+h_{\text {out }}^{\prime 2}\right)^{1 / 2}} .
$$

The corresponding outer solution $h_{\text {out }}(x)$ is that of a meniscus of a liquid reservoir joining the fiber surface. The contact angle of the meniscus at the surface is denoted as the apparent contact angle, $\theta_{a p}$, since it refers to the apparent angle on the scale of the outer solution. The boundary conditions therefore are:

$$
\begin{aligned}
& h_{\text {out }}(x=0)=0, \\
& h_{\text {out }}^{\prime}(x=0)=\theta_{a p}, \\
& h_{\text {out }}^{\prime}(x=\Delta)=\infty .
\end{aligned}
$$

For the present analysis we require only the asymptotic behavior near the contact line, which is obtained by a Taylor expansion,

$$
h_{\text {out }}(x)=\theta_{a p} x+\frac{1}{2} \kappa_{a p} x^{2}+\mathscr{O}\left(x^{3}\right) .
$$

Note that we consider small $\theta_{a p}$, since the inner solution is obtained in the lubrication limit. 
In general, the governing equation (3.14) cannot be solved analytically. In the following we will consider two extreme cases for which analytical solution can be obtained, namely the larger fiber radius case $\left(r_{0} \gg 1\right)$ and the small fiber radius case $\left(r_{0} \ll 1\right)$.

\section{Large fiber radius: $r_{0} \gg 1$}

In the case where the fiber radius is much larger than the capillary length, the second term on the right hand side of (3.15) due to the curvature of the fiber can be neglected. Then (3.14) can be written as

$$
\frac{h_{\text {out }}^{\prime \prime}}{\left(1+h_{\text {out }}^{\prime 2}\right)^{3 / 2}}=\Delta-x
$$

Integrating (3.20) once with respect to $x$, we obtain

$$
1-\frac{h_{\text {out }}^{\prime}}{\left(1+h_{\text {out }}^{\prime 2}\right)^{1 / 2}}=\frac{1}{2}(\Delta-x)^{2},
$$

where the boundary condition $h_{\text {out }}^{\prime} \rightarrow \infty$ at the position of the reservoir $(x=\Delta)$ is used. Evaluating (3.21) at the contact line position $(x=0)$ and using the geometrical connection $\sin \theta=h_{\text {out }}^{\prime} / \sqrt{1+h_{\text {out }}^{\prime 2}}$, we end up with

$$
\begin{aligned}
\Delta & =\sqrt{2\left(1-\sin \theta_{a p}\right)} \\
& \simeq \sqrt{2}\left(1-\theta_{a p} / 2\right)
\end{aligned}
$$

and (3.14) immediately gives

$$
\kappa_{a p} \simeq \sqrt{2}\left(1-\theta_{a p} / 2\right)
$$

\section{Small fiber radius: $r_{0} \ll 1$}

For thin fibers it has been shown that the outer region can be further divided into two subregions [37], as has been sketched in Fig. 3.2. In the region far away from the fiber $(h \gg 1)$, the term due to the curvature of the fiber can be neglected. On the other hand, gravity can be neglected in the region close to the fiber $(h \ll 1)$, and the meniscus is determined by the balance between the two curvature terms in (3.15). The profile near the fiber is a classical zero curvature interface that can be expressed as 


$$
r_{0}\left[\cosh \left(\frac{x}{r_{0} \cos \theta_{a p}}\right)+\sin \theta_{a p} \sinh \left(\frac{x}{h_{0} \cos \theta_{a p}}\right)-1\right]
$$

In the following paragraphs we will match this small-scale part of the outer solution to the viscous inner solution. We therefore make a Taylor expansion, for small values of $\theta_{a p}$,

$$
h_{\text {out }}=\theta_{a p} x+\frac{1}{2 r_{0}} x^{2}+\mathscr{O}\left(x^{3}\right) .
$$

To express our results in terms of the meniscus rise $\Delta$, we quote the result obtained by James [37] in which the two subregions of the outer meniscus were matched:

$$
\Delta=r_{0}\left[\ln \left(\frac{4}{r_{0}\left(1+\sin \theta_{a p}\right)}\right)-c\right],
$$

where $\mathrm{c}$ is Euler's constant $(0.57721 \ldots)$.

\subsubsection{Matching}

We are now in a position to perform the matching between inner and outer solutions. First, we write the inner solution in terms of the original variables,

$$
h_{\mathrm{in}}(x)=\delta^{1 / 3}\left[\frac{\kappa_{y} \theta_{e}^{2} x^{2}}{6 \lambda_{s}}+b_{y} \theta_{e} x+\mathscr{O}(1)\right] .
$$

Once more, we separately discuss the limits of large and small fiber radii.

\section{Large fiber radius: $r_{0} \gg 1$}

Comparing the inner solution (3.28) to the outer solution $(3.19,3.24)$ one finds the matching conditions

$$
\begin{aligned}
\theta_{a p} & =\delta^{1 / 3} b_{y} \theta_{e} \\
2-\theta_{a p} & =\sqrt{2} \delta^{1 / 3} \frac{\kappa_{y} \theta_{e}^{2}}{3 \lambda_{s}} .
\end{aligned}
$$




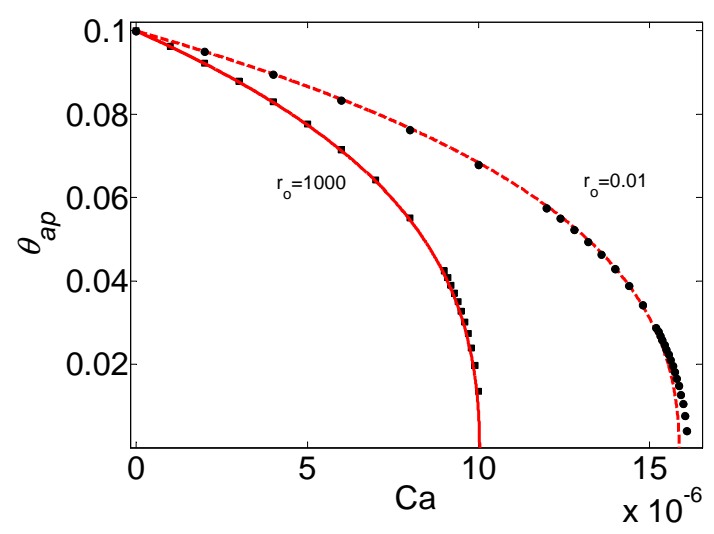

Figure 3.3: Apparent contact angle $\theta_{a p}$ versus $\mathrm{Ca}\left(\lambda_{s}=10^{-8}, \theta_{e}=0.1\right.$ radian) for large radius $\left(r_{0}=1000\right)$ and small radius $\left(r_{0}=0.01\right)$. Curves: result from asymptotic matching, solid curve: $r_{0}=1000$, dashed curve: $r_{0}=0.01$; Symbols: numerical result, squares: $r_{0}=1000$, circles: $r_{0}=0.01$.

Adding these two conditions leads to an equation for $s_{1}$ as a function of $\delta$ :

$$
\frac{2 / \theta_{e}}{\delta^{1 / 3}}+\frac{2^{2 / 3} A i^{\prime}\left(s_{1}\right)}{A i\left(s_{1}\right)}=\frac{2^{1 / 6} \exp [-1 /(3 \delta)]}{3 \pi A i^{2}\left(s_{1}\right) \lambda_{s} / \theta_{e}} .
$$

Once $s_{1}$ is known, one can compute the apparent contact angle

$$
\frac{\theta_{a p}}{\theta_{e}}=\frac{-2^{2 / 3} \delta^{1 / 3} A i^{\prime}\left(s_{1}\right)}{A i\left(s_{1}\right)} .
$$

A typical result for $\theta_{a p}$ as a function of $\mathrm{Ca}$ is shown in Fig. 3.3 (solid curve: $\left.r_{0}=1000\right)$. At vanishing speed, one recovers the equilibrium contact angle $\left(\theta_{e}=0.1\right.$ in this example). The apparent contact angle decreases for increasing speed, and tends to $\theta_{a p}=0$ at a critical value $\mathrm{Ca}_{c}$. The prediction from the matching compares very well to direct numerical solution of the problem, which will be discussed in the following section (solid squares). Of course, it is also possible to determine the critical speed directly from (3.31), as shown in [11]. The critical value $\delta_{c}$ is obtained when the Airy function takes its global maximum, $A i^{\prime}\left(s_{1}\right)=0$, corresponding to $s_{\max }=-1.088 \cdots$. This gives a critical speed

$$
\delta_{c}=\frac{1}{3}\left[\ln \left(\frac{\delta_{c}^{1 / 3} \theta_{e}^{2}}{2^{5 / 6} 3 \pi\left(A i\left(s_{\max }\right)\right)^{2} \lambda_{s}}\right)\right]^{-1} .
$$


Note that $\delta=3 \mathrm{Ca} / \theta_{\mathrm{e}}^{3}$ and $A i\left(s_{\max }\right)=0.53566 \ldots$. Physically, this corresponds to a vanishing apparent contact angle, as can be seen from (3.32) since $A i^{\prime}\left(s_{1}\right)=0$. Indeed, this confirms the conjecture by Derjaguin and Levi [24] that the maximum speed is attained when $\theta_{a p}=0$.

\section{Small fiber radius: $r_{0} \ll 1$}

We now perform a similar analysis for thin fibers by using the outer solution (3.26), which was never worked out previously. Comparing this to the inner solution (3.28) one finds the matching conditions

$$
\begin{gathered}
\theta_{a p}=\delta^{1 / 3} b_{y} \theta_{e}, \\
\frac{1}{r_{0}}=\delta^{1 / 3} \frac{\kappa_{y} \theta_{e}^{2}}{3 \lambda_{s}} .
\end{gathered}
$$

The parameter $s_{1}$ can be solved as function of $\delta$ from (3.35). More explicitly, we can write (3.35) as

$$
\frac{2 / \theta_{e}}{\delta^{1 / 3}}=\frac{2^{2 / 3} r_{0} \exp [-1 /(3 \delta)]}{3 \pi A i^{2}\left(s_{1}\right) \lambda_{s} / \theta_{e}}
$$

The apparent contact angle follows from (3.34). Since this condition is the same for both small fiber radius and large fiber radius, the explicit form of $\theta_{a p}$ is also given by eqn. (3.32).

Once again, solutions of the matching conditions cease to exist at a critical speed, which occurs when the Airy function takes its global maximum, $A i^{\prime}\left(s_{1}\right)=0$. In perfect analogy to the flat plate case, this corresponds to $\theta_{a p}=0$. The critical speed is given by

$$
\delta_{c}=\frac{1}{3}\left[\ln \left(\frac{r_{0} \delta_{c}^{1 / 3} \theta_{e}^{2}}{2^{1 / 3} 3 \pi\left(A i\left(s_{\max }\right)\right)^{2} \lambda_{s}}\right)\right]^{-1} .
$$

This result has the same structure as (3.33), valid for $r_{0} \gg 1$. Apart from numerical coefficients, the main difference is that the fiber radius $r_{0}$ appears inside the logarithm as the relevant outer length scale; for the flat plate the outer scale is the capillary length.

This result is further illustrated in Fig. 3.3 showing $\theta_{a p}$ for a radius $r_{0}=10^{-2}$ (dashed curve). The curve is similar to that obtained for a plate of infinite radius, with a vanishing contact angle at the critical point. Note that this critical speed depends 
weakly (logarithmically) on the fiber radius, in agreement with prediction (3.37). In addition, there is a also a logarithmic dependence of $\delta_{c}$ on the equilibrium contact angle $\theta_{e}$. Let us emphasize that the validity of the asymptotic analysis requires $\lambda_{s} / \theta_{e}$ to be small. This means that, strictly speaking, we cannot deal with extremely small values of $\theta_{e}$.

It is instructive to compare our results with Voinov's formula [27]. The prediction by Voinov for $\delta_{c}$ has the same structure as ours, but the factor inside the logarithm is not precisely specified (a ratio between the macroscopic length scale and the microscopic scale). In fact the factor reflects the dependence on the specific geometry of the problem, that in our approach is determined by the matching of the inner region and the outer region. Naturally, the inner scale turns out to be the slip length, while the outer scale is the fiber radius or the capillary length. However, Voinov's formula misses details like the factors $\theta_{e}, \delta_{c}$ inside the logarithm. Also the resulting $\theta_{a p}$ vs $\mathrm{Ca}$ is a bit different from Voinov's formula, as was previously discussed in detail by Eggers [11].

\subsection{Numerical solution}

We now perform a numerical analysis of the fiber withdrawal problem. This will confirm the validity of the asymptotics and extend the results to $r_{0} \sim 1$. However, the main added value is that the numerical solution can determine the complete bifurcation diagrams of dewetting for arbitrary $r_{0}$. These contain steady state solutions above $\Delta_{\max }$ that serve as transients towards film deposition [13, 16], and thus provide crucial additional information. Below we first develop a lubrication model that accounts for the axisymmetric nature of the flow. This quantitative correction with respect to the flat plate will turn out important for the bifurcation diagram. We then summarize the numerical results.

\subsubsection{Lubrication approximation on a fiber}

To formulate a hydrodynamic model for the axisymmetric meniscus on a fiber, we consider Stokes equations

$$
\begin{gathered}
-\vec{\nabla} p+\eta \nabla^{2} \vec{U}-\vec{\nabla} \Phi=0, \\
\vec{\nabla} \cdot \vec{U}=0
\end{gathered}
$$


where $p$ is the pressure field in the liquid, $\eta$ is the viscosity of the liquid, $\vec{U}$ is the velocity field in the frame comoving with the fiber, and $\Phi$ is the gravitational potential per unit volume in the liquid. Since the meniscus is axisymmetric, the velocity in azimuthal direction is zero. We consider small contact angle, $\theta_{e} \ll 1$, thus the flow is mainly in the vertical $x$ direction, namely, the radial component of velocity is much smaller than the vertical component (i.e. $\left|U_{r}\right| \ll\left|U_{x}\right|$ ). The flow is solved with a no-stress condition at the interface, is located at $r=r_{0}+h$, and reads (in the frame of the fiber)

$$
\left.\eta\left(\frac{\partial U_{r}}{\partial x}+\frac{\partial U_{x}}{\partial r}\right)_{r=r_{0}+h} \approx \eta \frac{\partial U_{x}}{\partial r}\right|_{r=r_{0}+h}=0 .
$$

At the fiber surface, $r=r_{0}$, we apply a Navier slip boundary condition

$$
\left.U_{x}\right|_{r=r_{0}}=\left.\lambda_{s} \frac{\partial U_{x}}{\partial r}\right|_{r=r_{0}}
$$

The axial (vertical) component of the velocity field then becomes

$$
\begin{array}{r}
U_{x}=\frac{1}{2 \eta} \frac{\partial(p+\Phi)}{\partial x} \\
\times\left[\frac{r^{2}-r_{0}^{2}}{2}-\left(r_{0}+h\right)^{2} \ln \left(\frac{r}{r_{0}}\right)-\lambda_{s}\left(2 h+\frac{h^{2}}{r_{0}}\right)\right]
\end{array}
$$

For thin films $h / r_{0} \ll 1$ this reduces to the usual parabolic Poiseuille profile, but quantitative corrections appear when $h / r_{0} \sim 1$.

The lubrication equation is obtained by imposing a zero flux condition in the frame of the reservoir

$$
\int_{r_{0}}^{r_{0}+h}\left(U_{x}+U_{0}\right) r d r=0 .
$$

With this, (3.42) can then be simplified as

$$
\frac{\partial(p+\Phi)}{\partial x}=\frac{3 \eta U_{0} f(d)}{h\left[h+3 \lambda_{s}(1+d / 2) f(d)\right]},
$$

where we introduced $d=h / r_{0}$ and

$$
f(d)=\frac{8 d^{3}(2+d)}{3\left[4(1+d)^{4} \ln (1+d)-d(2+d)\left(2+6 d+3 d^{2}\right)\right]} .
$$


This function is a correction factor with respect to the flat plate $(d=0)$, and has the property $f(0)=1$. Finally, we replace the pressure by the Young-Laplace equation,

$$
p-p_{0}=-\gamma \kappa,
$$

where $\kappa$ is the curvature of the interface given by (3.15). This gives the lubrication equation on a fiber

$$
\frac{\partial \kappa}{\partial x}=\frac{3 \mathrm{Ca} f(d)}{h\left[h+3 \lambda_{s}(1+d / 2) f(d)\right]}-1 .
$$

Note that once again all lengths are scaled by the capillary length. For $d=h / r_{0} \ll 1$, we recover the usual lubrication equation since $f(0)=1$.

\subsubsection{Results}

\section{Critical speed}

The above lubrication equation (3.47) is solved numerically with boundary conditions

$$
\begin{aligned}
h(0) & =0 \\
h^{\prime}(0) & =\theta_{e}
\end{aligned}
$$

imposed at the contact line and

$$
\begin{aligned}
h^{\prime}(\Delta) & =\infty, \\
\kappa(\Delta) & =0,
\end{aligned}
$$

at the reservoir. We varied $r_{0}$ and $\lambda_{s}$ and determined the meniscus as a function of $\mathrm{Ca}$.

Figure 3.4 shows the meniscus rise $\Delta$ as a function of $\mathrm{Ca}$ on a fiber of radius $r_{0}=10^{-2}$. Different symbols correspond to different values of the slip length. In all cases we find a critical $\mathrm{Ca}_{\mathrm{c}}$ above which solutions cease to exist. This indeed occurs close to $\Delta_{\max }$ corresponding to a vanishing $\theta_{a p}$, which is indicated by the horizontal dotted line. The curves are the predictions from the matched asymptotics, showing a good agreement with the numerical solutions. In particular, one observes convergence as the slip length is reduced from $\lambda_{s}=10^{-6}, 10^{-7}$ to $10^{-8}$. This is because the separation of the two length scales $\lambda_{s}$ and $r_{0}$ is enhanced, which improves the validity of the matching asymptotic expansion. The same results were previously reported in Fig. 3.3, expressed in terms of $\theta_{a p}$ rather than $\Delta$. 


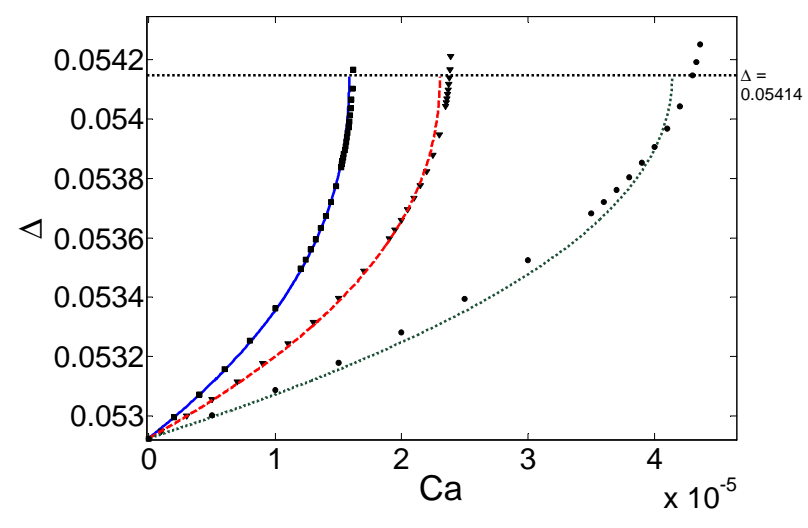

Figure 3.4: Height of meniscus $\Delta$ versus speed for different slip lengths $\left(r_{0}=10^{-2}\right.$, $\theta_{e}=0.1$ radian). Curves are results from asymptotic matching, solid curve (the most left curve): $\lambda_{s}=10^{-8}$, dashed curve: $\lambda_{s}=10^{-7}$, dotted curve (the most right curve): $\lambda_{s}=10^{-6}$. Symbols are the corresponding numerical results. As the slip length gets smaller, the agreement between numerics and asymptotic matching becomes better. The horizontal dotted line indicates the maximum height of meniscus $\Delta=0.05414$ calculated by (3.27) with $\theta_{a p}=0$.

It is interesting to show how the critical speed $\mathrm{Ca}_{c}$ depends on the fiber radius $r_{0}$. The numerical results are plotted as squares in Fig. 3.5. In agreement with the asymptotic analysis one observes two regimes. At small radii, $r_{0} \ll 1$, the critical speed depends logarithmically on the radius. The solid red line is the asymptotic result (3.37). For large radii the speed approaches the value of the flat plate (3.33), indicated as dashed black line. Indeed, the cross-over occurs for fibers with a radius that is comparable to the capillary length $r_{0} \sim 1$.

\section{Meniscus rise: bifurcation diagram}

The results shown in Fig. 3.4 represent only the lowest branch of solutions of a more complete bifurcation diagram. Indeed, one can identify solutions with $\Delta$ extending to arbitrary height above the meniscus, which are all characterized by $\mathrm{Ca}<\mathrm{Ca}_{\mathrm{c}}$. These are summarized in Figs. 3.6ace for different fiber radii (all curves correspond to $\theta_{e}=0.05$ and $\lambda_{s}=10^{-5}$ ). For $r_{0}=1000$ (Fig. 3.6a), we see after reaching $\mathrm{Ca}_{\mathrm{c}}$, the curve turns back to $\mathrm{Ca}<\mathrm{Ca}_{\mathrm{c}}$ but with solutions of increasing $\Delta$. We refer to these solutions as the second branch, which is known to be unstable [13]. Further upwards we observe a series of bifurcations to higher branches, oscillating around 


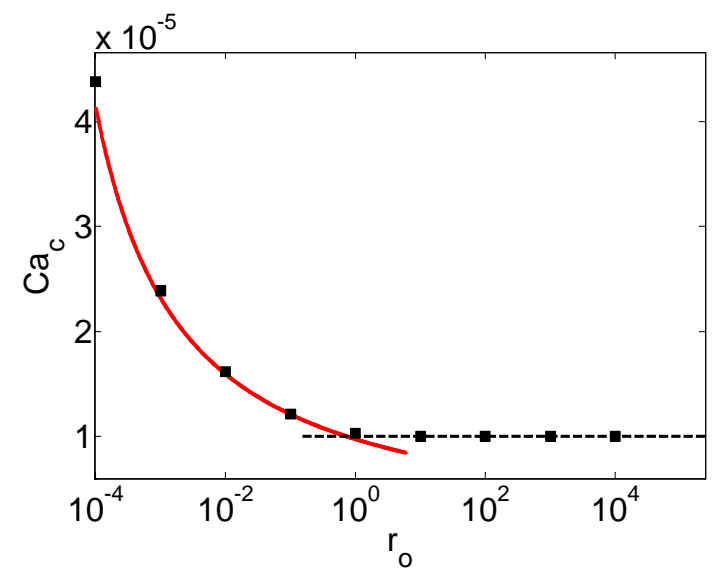

Figure 3.5: Critical capillary number $\mathrm{Ca}_{\mathrm{c}}$ versus fiber radius $r_{0}\left(\theta_{e}=0.1\right.$ radian, $\left.\lambda_{s}=10^{-8}\right)$. Squares: numerical results; Curve: Result from asymptotic matching for small fiber radius [Eq. (3.37)]; Dotted line: Result from asymptotic matching for large fiber radius [Eq. (3.33)].

a characteristic value $\mathrm{Ca}^{*}$. Typical meniscus profiles are shown in Fig. $3.6 \mathrm{~b}-$ in order to compare the profiles we have shifted the positions of the contact line such that the baths collapse. Following the bifurcation diagram, the profiles evolve to a film solution for which the contact line has moved to arbitrary height above the meniscus, i.e. $\Delta \rightarrow \infty$. This film solution (shown in Fig. 3.6b) is not the LandauLevich-Derjaguin film, but corresponds to the new class of "thick film" solutions identified in [15].

We then decrease the fiber radius to $r_{0}=0.1$, as shown in Figs. 3.6cd. We find that $\mathrm{Ca}_{\mathrm{c}}$ increases almost by a factor 2 with respect to the large radius. By contrast, $\mathrm{Ca}^{*}$ corresponding to the thick film increases only by a small amount. As a result the values of $\mathrm{Ca}_{\mathrm{c}}$ and $\mathrm{Ca}^{*}$ have become more separated. Also, the corresponding meniscus profiles display more structure. The thick film exhibits much stronger oscillations before joining the reservoir. These trends becomes more dramatic up further decreasing the radius $r_{0}=0.001$ (Figs. 3.6ed). The difference between $\mathrm{Ca}_{\mathrm{c}}$ and $\mathrm{Ca}^{*}$ is very pronounced, and $\Delta$ changes much more dramatically for the 3 rd branch solutions. In this sense, the bifurcation diagram has a very different structure from those of large fiber radius. Interestingly, there still exists a thick film solution matching to the bath, but the profile displays many oscillations (Fig. 3.6f). These oscillations decay only very slowly when moving further away from the bath - the asymptotic thickness of 
the film is indicated by the horizontal line just above $x$ axis. While for the flat plate the thick film solutions have been observed experimentally [15], we expect the oscillatory solution obtained for small radii to be unstable and of no physical relevance.

For completeness, we report the values of $\mathrm{Ca}_{c}$ and $\mathrm{Ca}^{*}$ for different radii in a separate graph (Fig. 3.7). Note that the theoretical curve for $\mathrm{Ca}_{\mathrm{c}}$ deviates from the numerical results as early as $r_{0} \lesssim 10^{-2}$. The reason is that here we use a realistic value for the slip length $\lambda_{s}=10^{-5}$ (corresponding to $\sim 10 \mathrm{~nm}$ ), instead of $\lambda_{s}=10^{-8}$ used in Fig. 3.5. Clearly, the scale separation required for the asymptotic analysis starts to break down when the ratio $\lambda_{s} / r_{0}$ is no longer very small.

\subsection{Discussion}

We investigated the steady-state profiles of axisymmetric menisci on a fiber that is withdrawn from a viscous liquid. The main motivation for this work was the mixed experimental observations on the transition to film deposition obtained for fibers, large cylinders and plates. Sedev \& Petrov [18] found that the maximum steady profile has a meniscus rise identical to a perfectly wetting liquid at equilibrium, suggesting a vanishing apparent contact angle $\theta_{a p}$. Other experiments found that steady-state solutions disappeared at a nonzero $\theta_{a p}[12,38]$, although the critical point could be accessed during transients [14]. Our present calculations show that steady solutions always cease to exist at $\theta_{a p}=0$, independent of the fiber radius. In addition, stability arguments put forward in $[11,48]$ suggest that all solutions of the lowest branch are perfectly stable up to the maximum speed, consistent with a saddle-node bifurcation [13]. In that sense, our results do not provide an explanation why experimentally it is practically impossible to achieve steady menisci closer to $\theta_{a p}=0$. The main effect that was not taken into account in our calculations is contact angle hysteresis due to heterogeneity of the substrate [49], which was previously suggested to affects the details of the transition [50]. It has remained a challenge, however, to incorporate this into a full hydrodynamic description of moving contact lines. Essentially one has to modify the boundary condition by imposing a time-dependent microscopic contact angle at the moving contact line.

The bifurcation diagrams calculated in the second part of this Chapter, however, do provide a new experimental perspective on the dynamics of film deposition. As shown in [14], such bifurcation diagrams may be probed experimentally as transient states during entrainment. Namely, for $\mathrm{Ca}>\mathrm{Ca}_{c}$ the evolution of the meniscus exactly follows the bifurcation diagram when plotting $\Delta$ versus the relative contact line velocity with respect to the solid. For very large fiber radius the profiles with large capillary rise are smoothly connected to the bath by a film that only displays a small 
"dimple" close to the bath. These dimple solutions have indeed been observed experimentally when plates are withdrawn with speeds above the critical speed. By contrast, for small fiber radii these solutions exhibit very strong oscillations (Fig. 3.6) and we expect these solutions to be very unstable. In that case another dynamical mode must appear in order to deposit a liquid film - for example, one could think of the classical dewetting rim at the contact line connected to a Landau-Levich film [51]. A further investigation of these transients above the critical speed, in particular for different radii, should give a more complete picture of the forced wetting transition. 

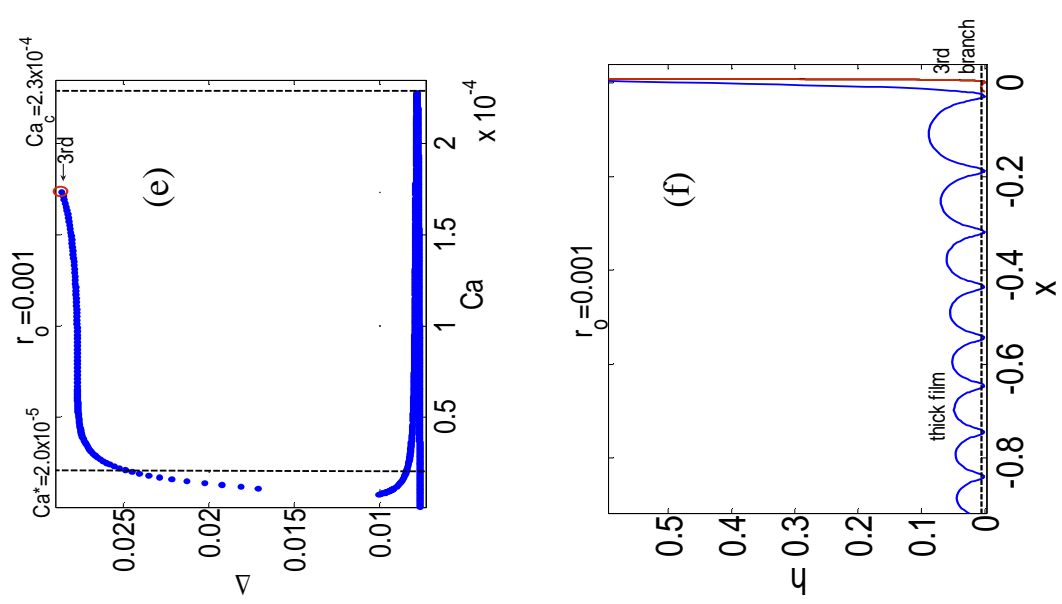

б
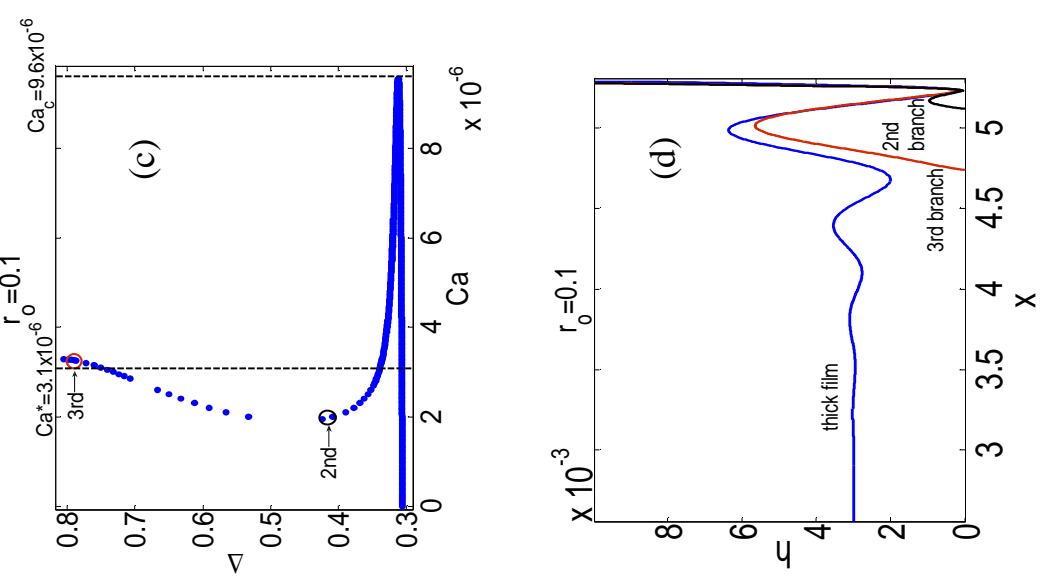

웅 $\Xi 8$

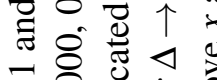

느응

80.000

II

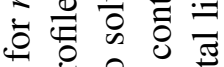

艺

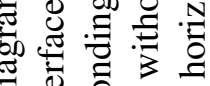

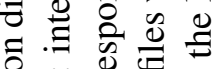

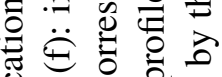

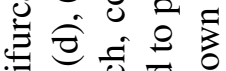

0 论

$\ddot{0} 0$

ड़

$\because$ च 00

ङ્

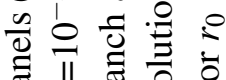

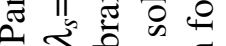

เి

웡 웡

政
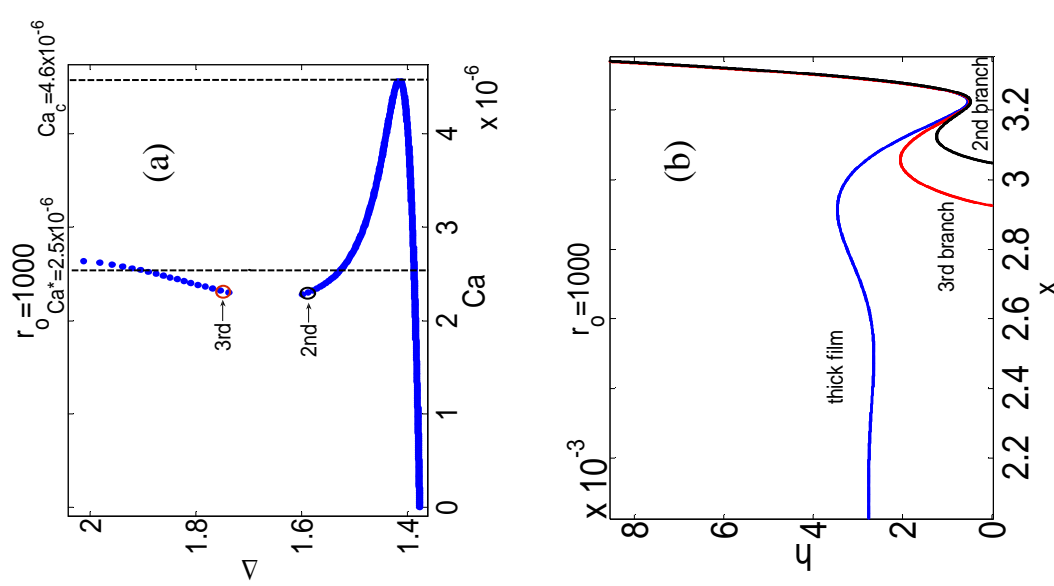

Ð

के

뭉

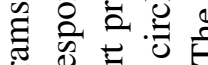

年

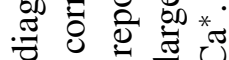

б 0

氜芯芯

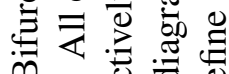

ต

$\ddot{\omega} \overrightarrow{0} \stackrel{0}{0} . \overline{0}$

过

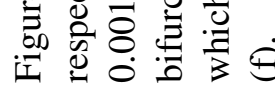




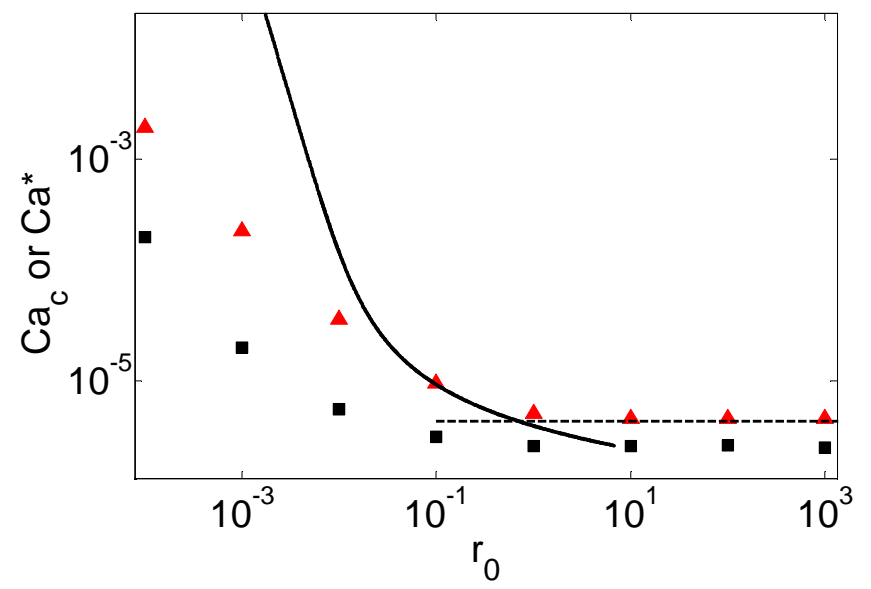

Figure 3.7: $\mathrm{Ca}_{\mathrm{c}}$ and $\mathrm{Ca}^{*}$ as function of $r_{0}$ for the same parameters used in Fig. 3.6. Triangles: numerical results for $\mathrm{Ca}_{c}$; Squares: numerical results for $\mathrm{Ca}^{*}$; Curve: Result for $\mathrm{Ca}_{\mathrm{c}}$ from asymptotic matching for small fiber radius [Eq. (3.37)]; Dotted line: Result for $\mathrm{Ca}_{\mathrm{c}}$ from asymptotic matching for large fiber radius [Eq. (3.33)]. 


\section{Air Entrainment by Viscous Contact Lines *}

The entrainment of air by advancing contact lines is studied by plunging a solid plate into a very viscous liquid. Above a threshold velocity, we observe the formation of an extended air film, typically 10 microns thick, which subsequently decays into air bubbles. Exploring a large range of viscous liquids, we find an unexpectedly weak dependence of entrainment speed on liquid viscosity, pointing towards a crucial role of the flow inside the air film. This induces a striking asymmetry between wetting and dewetting: while the breakup of the air film strongly resembles the dewetting of a liquid film, the wetting speeds are larger by orders of magnitude.

\subsection{Introduction}

Objects that impact on a liquid interface can entrain small bubbles of air into the liquid. This happens for example when raindrops fall in the ocean [52] or when liquid is poured into a reservoir at sufficiently large speeds [20,53]. Such entrainment of air is often a limiting factor in industrial applications such as coating and nano-scale printing techniques, where the bubbles disturb the process $[2,54]$. A well studied case is the entrainment of air by very viscous jets impacting on a reservoir of the same liquid $[20,53,55,56]$. The onset of entrainment is essentially determined by the

${ }^{*}$ Published as: A. Marchand, T.S. Chan, J.H. Snoeijer and B. Andreotti, Air entrainment by contact lines of a solid plate plunged into a viscous fluid, Phys. Rev. Lett. 108, 204501 (2012). 
properties of the liquid, $U_{e} \sim \gamma / \eta_{\ell}$, which reflects a balance of the liquid viscosity $\eta_{\ell}$ and the surface tension $\gamma$. Changing the nature of the gas only has a minor influence on the entrainment process $[20,53]$.

A very different picture has emerged recently in the context of drops impacting on a wall, for which the presence of air has a dramatic effect [22]. It was found that splashing can be suppressed completely by reducing the air pressure to about a third of the atmospheric pressure. This caused huge excitement [57-61], in particular because such a pressure reduction does not lead to any change of the gas viscosity $\eta_{g}$ : pressure only affects the gas density, and thus the speed of sound and the mean free path in the gas. A similar paradox is encountered for air entrainment by rapidly advancing contact lines, where a liquid advances over a surface that it partially wets [2, 23, 39, 62-64]. Once again, it was found that depressurizing the gas leads to a significant increase of the threshold of air entrainment [23,65]. This contradicts the classical viewpoint that, for given wettability, the contact line speed depends mainly on the liquid properties as $\sim \gamma / \eta_{\ell}[9,12,21,39]$, with minor influence of the gaseous phase.

In this Chapter we reveal the role of the air for advancing contact lines in a paradigmatic system: a partially wetting solid plate is plunged into a reservoir of viscous liquid. The typical experimental scenario is presented in Fig. 4.1. When the plate is plunged at small speeds, the contact line equilibrates to form a stationary meniscus and no air is entrained into the liquid (Fig. 4.1a). Above a critical velocity, however, the contact line keeps moving downward into the reservoir and deposits a film of air (Figs. $4.1 \mathrm{bc}$ ), typically 10 microns thick. The substrate is sufficiently clean and the vibrations sufficiently low to avoid contact line pinning effects. The air film rapidly destabilizes after its formation, before ultimately decaying into small air bubbles (see Supplementary Material section). The liquid viscosity $\eta_{\ell}$ is varied over more than two decades by using silicon oils of different molecular weights. It is found that the entrainment speed $U_{e}$ changes much less than the expected scaling $\sim 1 / \eta_{\ell}$. Using an approximate hydrodynamic model we argue that this can be attributed to the flow of air into the strongly confined film, making the contact line velocity strongly dependent on both gas and liquid viscosities. This induces a striking asymmetry between wetting and dewetting: a liquid can advance much faster than it recedes, by orders of magnitude.

\subsection{Experimental setup and results}

We explore how this process of air entrainment is influenced when varying the liquid viscosity. We therefore use silicon oils (PDMS, Rhodorsil 47V series) with dynamic 


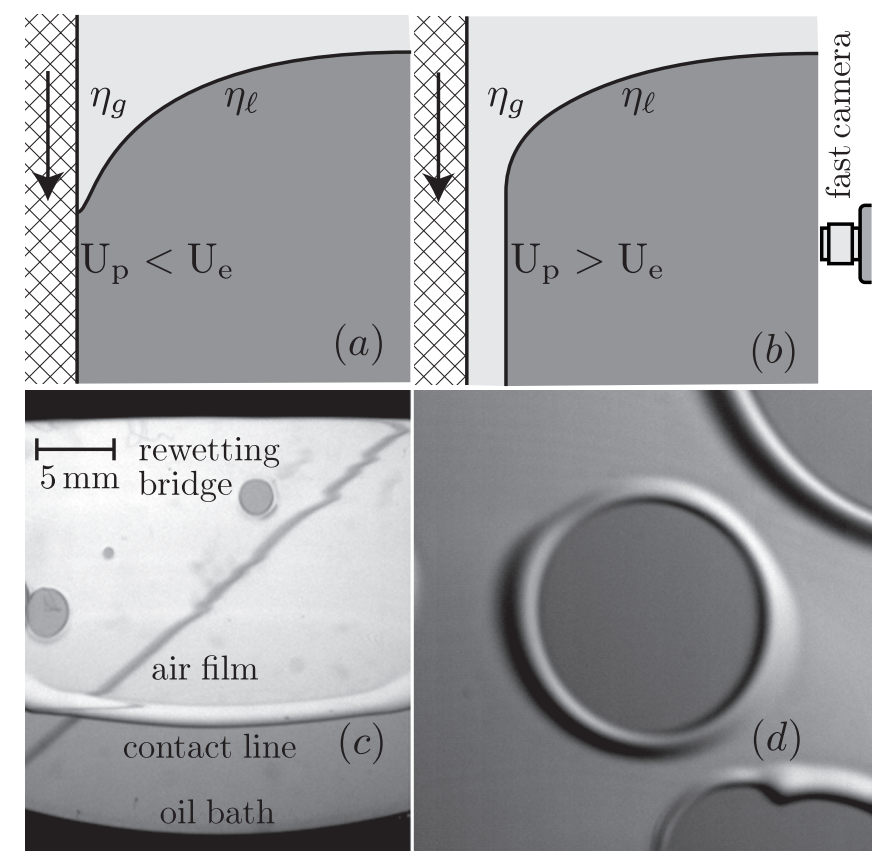

Figure 4.1: Air entrainment by a contact line when a solid plate is plunged into a viscous liquid. (a) Sketch of dynamical meniscus below the threshold of air entrainment, and (b) above the threshold of air entrainment, where a film of thickness $h$ develops. A high speed camera is placed perpendicularly to the substrate. (c,d) Front views of the air film entrained into a viscous silicone oil ( $\left.\eta_{\ell}=0.1 \mathrm{~Pa} . \mathrm{s}\right)$. (c) The extended air film is formed behind the contact line and moving downwards into the bath (taken $70 \mathrm{~ms}$ after plunging). The film is destroyed by the formation of "rewetting" bridges. The diagonal dark line is the reflection of a straight wire that gives an impression of the interface profile. (d) Zoom of the "rewetting" bridge, which is the inverse of the dewetting of a liquid film. Once the oil reestablishes contact with the solid, a growing circular zone invades the air film.

viscosities $\eta_{\ell}=0.02,0.10,0.5,1.0$ and 5 Pa.s. These liquids are essentially nonvolatile, insensitive to contamination, while the surface tension $\gamma=22 \mathrm{mN} \cdot \mathrm{m}^{-1}$ and density $\rho=980 \mathrm{~kg} . \mathrm{m}^{-3}$ are approximately constant for all viscosities. The reservoir containing the liquid is a transparent acrylic container of size $29 \times 15 \times 13.5 \mathrm{~cm}$, which is much larger than the capillary length $\ell_{\gamma}=(\gamma / \rho g)^{1 / 2}=1.5 \mathrm{~mm}$. The substrate consists of a silicon wafer (circular, diameter $10 \mathrm{~cm}$ ), which is coated by a thin layer of fluorinated material (FC 725 (3M) in ethyl acetate). For all liquids, this results into static contact angles between $51^{\circ}$ and $57^{\circ}$. The wafer is clamped onto a $10 \mathrm{~mm}$ thick 
metallic blade screwed to a $50 \mathrm{~cm}$ long high-speed linear stage. The combination of controlled speeds and very viscous liquids avoids complexities of splashing as well as the formation of interface cusps [2, 23, 39, 63, 64]. In addition the effect of inertia is eliminated both in the gas and in the liquid: the Reynolds number based on the film thickness $h$ and on the entrainment threshold $U_{e}$ is at worst $\sim 0.2$, but typically orders of magnitude smaller. For each liquid, we plunge the wafer into the reservoir at different plate velocities $U_{p}$, up to $0.7 \mathrm{~m} / \mathrm{s}$. The process is recorded using a highspeed Photron SA3 camera $(2000 \mathrm{~Hz}, 1024 x 1024$ pixels). The contact line velocities are extracted from space-time diagrams using a correlation technique with a subpixel resolution, leading to a precision within a percent. Reproducibility is within $15 \%$. The film thickness $h$ is determined by dividing the volume of air entrained in the bath by the surface of the film (see experimental methods in the Supplementary Material). The resulting thickness is reported in Fig. 4.2c for a fixed plate velocity, and is of the order of $10 \mu \mathrm{m}$ for all viscosities.

Interesting dynamical structures are observed during air entrainment (Fig. 4.1c). At the front of the film, the contact line develops a ridge-like shape that is common for dewetting of liquid films [51, 66, 67]. The peculiarity of the present experiment is that in this case the air is dewetted, not the liquid. An even more striking analogy with classical dewetting of liquids is the nucleation of nearly circular regions inside the film (Fig. 4.1d). However, the circles now represent regions of rewetting, where the liquid reestablishes the contact with the solid [68]. These "rewetting bridges" can be considered as the inverse of the "dewetting holes", since the roles of air and liquid are exchanged. The bridges form nearly perfect circles, apart from the two largest viscosities for which they are slightly stretched vertically; bridges that form after the plate is stopped are circular for all $\eta_{\ell}$. The radius of the bridge increases linearly with time, and the advancing contact lines collects the air inside a thick rim. While this is analogous to the inverse problem of the dewetting holes, the process is by no means symmetric: the rewetting circles grow with a velocity $U_{e}$ that is orders of magnitude faster than their dewetting counterparts, up to a factor 1000 for the liquids used in this study.

We further quantify the velocity of air entrainment for different liquid viscosities. A first measurement of $U_{e}$ is obtained from the growth velocities of the rewetting bridges as in Fig. 4.1d. To ensure perfectly circular bridges, measurements are done immediately after stopping the plate. A second entrainment velocity is given by the plate velocity at which the film first appears. Since by definition, the contact line hardly moves downward at the transition, this velocity is most accurately determined from experiments well above the transition according to the following principle. By selecting a central part of the front of the air film, we first obtain the contact line 

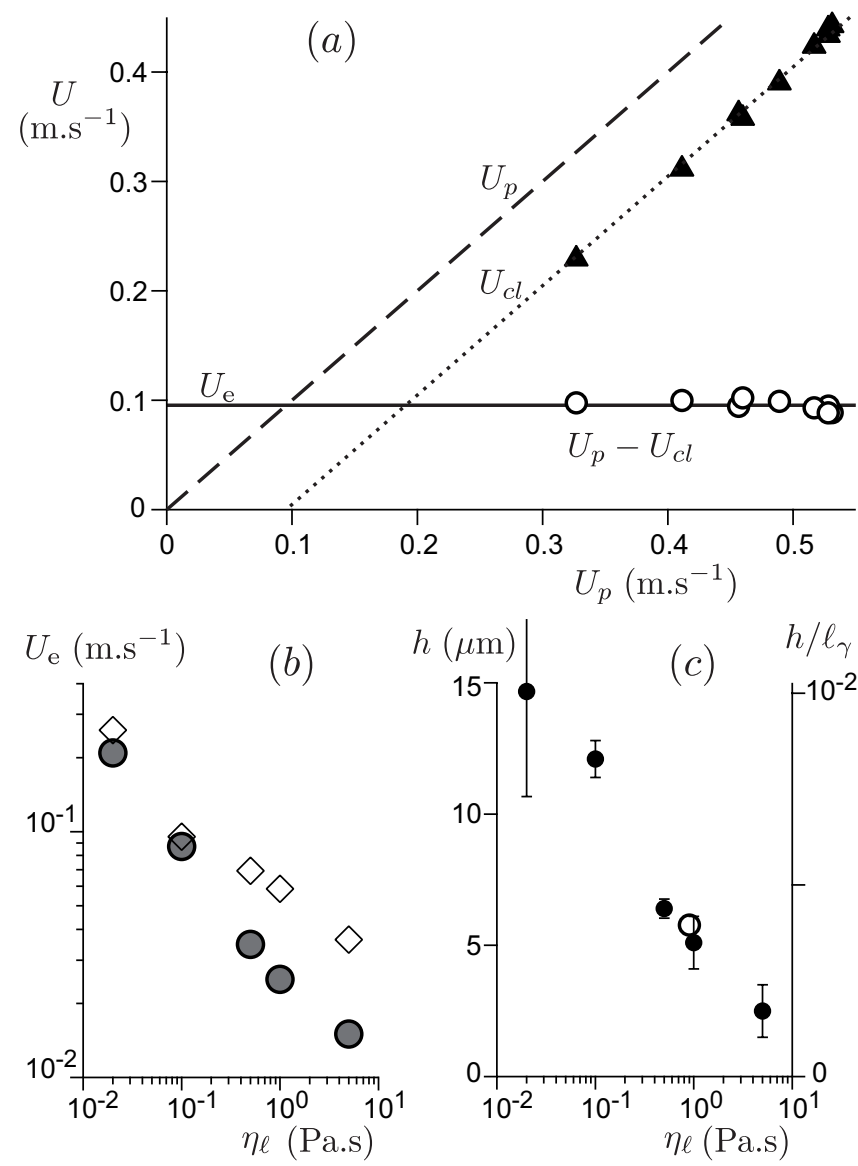

Figure 4.2: (a) Contact line velocity $U_{c l}$ (triangles) as a function of the plate velocity $U_{p}$, for $\eta_{\ell}=0.1$ Pa.s. The relative velocity $U_{p}-U_{c l}$ (circles) is independent of the plate speed. (b) Entrainment speed $U_{e}$ for different liquid viscosities $\eta_{\ell}$, measured in two ways: the diamonds represent the relative velocity with respect to the plate, $U_{p}-U_{c l}$, while the circles are the bridge rewetting speeds (see Fig. 4.1d). (c) Film thickness $h$ for different $\eta_{\ell}$, taken at constant plate velocity $U_{p}=0.67 \mathrm{~m} . \mathrm{s}^{-1}$. The open circle was taken for a liquid jet of glycerol entraining air at the same speed [53]. The error bars are determined from a set of independent measurements.

velocity $U_{c l}$ in the frame of the liquid reservoir. Figure 4.2a reports the measured values for $\eta_{\ell}=0.1 \mathrm{~Pa}$.s, showing that the contact line velocity increases linearly with plate velocity $U_{p}$. Interestingly, however, the relative velocity, $U_{e}=U_{p}-U_{c l}$, turns out to be independent of the plate velocity. Clearly, the film can only develop if the contact line can propagate downwards, i.e. when $U_{c l}>0$ or $U_{p}>U_{e}$, thus provid- 


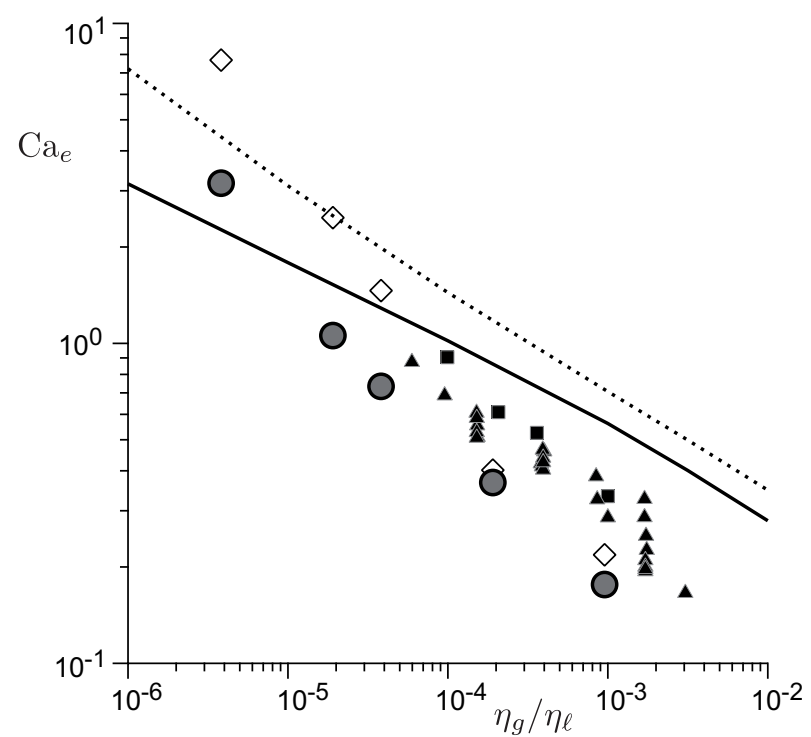

Figure 4.3: Dimensionless entrainment speed, $\mathrm{Ca}_{e}=U_{e} \eta_{\ell} / \gamma$, versus the viscosity ratio $\eta_{g} / \eta_{\ell}$ for: silicon-oil/air $(\bullet, \diamond$, present data), silicon-oil/air ( $\boldsymbol{\square}$ after [23]) and various liquids/air ( $\boldsymbol{\Lambda}$ after [69]). Curves: numerical results discussed in the Supplementary Material. Corresponding parameters: oil slip lengths $\lambda_{\ell}=10^{-5} \ell_{\gamma}$, and air slip lengths $\lambda_{g}=10^{-4} \ell_{\gamma}$ (solid line) and $10^{-2} \ell_{\gamma}$ (dotted line), corresponding to mean free paths $\ell_{\mathrm{mfp}}=70 \mathrm{~nm}$ (solid) and $7 \mu \mathrm{m}$ (dotted). The theoretical curves do not suggest a definite exponent.

ing an accurate determination of the critical speed. In analogy to receding contact lines this velocity appears to be an intrinsic property of the advancing contact line, independent of $U_{p}$ [12]. In other words, the structure of the contact line and the film appear to be completely independent from the bath: only the velocity relative to the plate matters. Indeed, the rewetting bridges display the same rim-like structure as the contact line front in Fig. 4.1c. This suggests that we may consider the growth velocity of the rewetting bridges to be an independent measurement of the entrainment velocity. The resulting entrainment velocities $U_{e}$ are shown in Fig. $4.2 \mathrm{~b}$, as a function of liquid viscosity $\eta_{\ell}$. Indeed, the two experimental definitions of $U_{e}$ agree very well for the smallest $\eta_{\ell}$ (open diamonds are based on the front of the film, closed circles correspond to rewetting bridges). For larger $\eta_{\ell}$, the film rapidly destabilizes and it is more difficult to define the front of the film. This induces a difference between the two types of velocity measurements of about a factor 2; the bridge velocities are certainly more reproducible in this very viscous regime.

The key result of the velocity measurements is that, although $U_{e}$ decreases with 
liquid viscosity, the dependence is clearly much weaker than the expected $\sim 1 / \eta_{\ell}$. The entrainment speed is reduced by a factor 10 , while viscosity is varied by a factor 250 (Fig. 4.2b). The data would be reasonably fitted by an exponent $-1 / 3$ or $-1 / 2$, but we refrain from claiming any definite power-law dependence. Since the liquid inertia is negligible for these highly viscous liquids, this means that the properties of the air must have a significant influence on the entrainment speed. On the other hand, the speed is not determined by the air alone, since that would yield no dependence on $\eta_{\ell}$ at all. To reveal the interplay between air and liquid phases, we introduce a dimensionless capillary number, $\mathrm{Ca}_{e}=U_{e} \eta_{\ell} / \gamma$, that is based on the liquid viscosity. The experimental results are represented in Fig. 4.3, showing $\mathrm{Ca}_{e}$ versus the ratio of gas and liquid viscosities $\eta_{g} / \eta_{\ell}$ (closed circles and open diamonds). Clearly, the capillary number for air entrainment displays a dependence that is much stronger than $\sim \ln \left(\eta_{\ell} / \eta_{g}\right)$, which is the scaling for air entrainment by liquid jets [20,53] and the prediction by Ref. [21]. The air thus has a much larger influence than expected. On the same figure we collected data from the coating literature, based on tapes running continuously in a bath, showing a similar trend (various symbols, see caption). Note that in these experiments the contact line typically develops a sharp cusp from which small air bubbles are emitted, rather than an extended air film. At present there is no detailed understanding of the conditions necessary to trigger such cusps. In the case of receding contact lines, however, the instability of cusps are known to give similar values for $\mathrm{Ca}_{c}$ as straight contact lines $[1,12,14]$.

\subsection{Interpretation and discussion}

How can one understand the influence of the air, despite its very small viscosity? The key lies in the geometry of the flow near the entrainment transition. In the receding case, where the plate is pulled from the bath, it is well-known that a liquid film appears whenever capillary forces can no longer balance the viscous drag. This transition occurs when the apparent, dynamic contact angle $\theta \rightarrow 0$ [3]. In this small contact angle limit, the rate of viscous dissipation scales as $\sim \eta_{\ell} U^{2} / h[9,39]$, where $h \simeq \theta x$ is the local thickness of the liquid, and $x$ the distance to the contact line. The $1 / h$ proportionality for the dissipation has two key consequences. First, the geometric confinement of liquid to a shallow wedge, as in Fig. 4.4a, enhances viscous effects due to the factor $1 / \theta$. Second, the dependence $\sim 1 / x$ shows that dissipation is largest at small scales. As a consequence, the integrated viscous dissipation is due to all length scales, with comparable contributions from each decade between nanometer and millimeter. In the advancing case, however, the flow direction is reversed and the interface bends towards an angle $\theta \rightarrow \pi$. Figure 4 .4b shows the classical solution for 

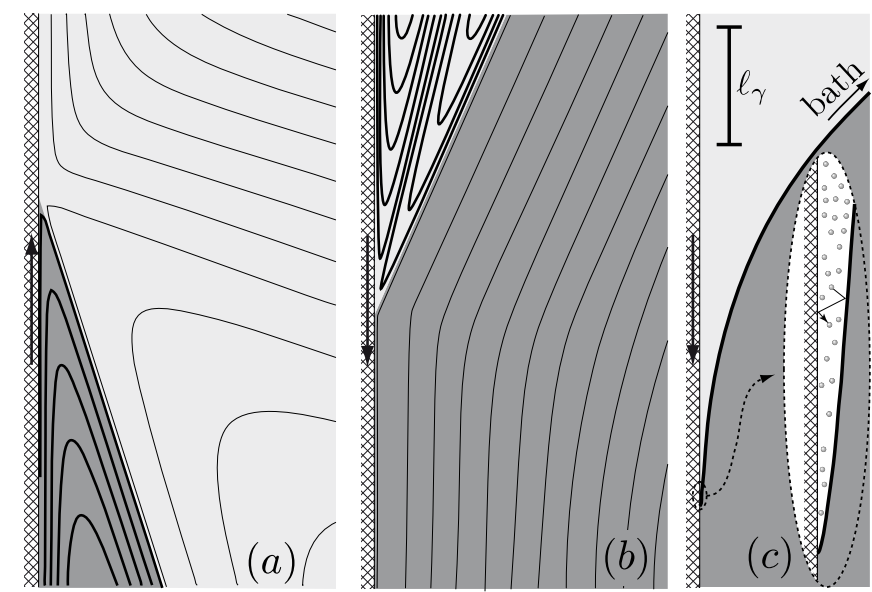

Figure 4.4: Streamlines in a perfect wedge [6] of angle $\theta$ for (a) a receding contact line (with $\theta$ close to 0 ), and for (b) an advancing contact line (with $\theta$ close to $\pi$ ). In the advancing case, the viscous dissipation in the gas phase will dominate over the liquid phase due to the strongly confined circulation in the gas wedge. (c) Shape of the curved interface near the entrainment threshold. In a range of thickness $h$ between the molecular scale and the scale $\ell_{\gamma}$ of the bath, the local angle $\theta$ is close to $\pi$ so that dissipation is dominated by air. Inset: When the mean free path $\ell_{\mathrm{mfp}}$ is comparable to $h$, then air behaves as a Knudsen gas.

a perfect wedge by Huh \& Scriven [6], illustrating that the flow of liquid becomes increasingly smooth when $\theta$ approaches $\pi$. In fact, it can be shown from these solutions that the liquid dissipation $\sim(\pi-\theta)^{2}$ for angles close to $\pi$ (see Supplementary Material). While this would enable arbitrarily large speeds when $\theta \rightarrow \pi$, this mechanism is counteracted by the flow in the remaining wedge of air. The air becomes increasingly confined to an angle $\pi-\theta$, and the associated gas dissipation scales as $\sim 1 /(\pi-\theta)$. Hence, even the smallest gas viscosity ultimately gives a lower bound on the dissipation, and thus an upper bound on the advancing speed.

A quantitative theory for air entrainment requires a more detailed description of the interface shape, which in reality is strongly curved (Fig. 4.4c). This means that the local angle $\theta$ should be considered to vary with $x$, and that Fig. 4.4b only provides a local estimate of the flow field. For air entrainment to occur, however, there must be a range of scales where the air flow is dominant, which from the above scaling arguments occurs when $(\pi-\theta)<\left(\eta_{g} / \eta_{\ell}\right)^{1 / 3}$. Since each decade provides a comparable dissipative contribution, this qualitatively explains why total dissipation involves both $\eta_{\ell}$ and $\eta_{g}$. We further model this in the spirit of [29, 70], extending the common lubrication approximation to large slopes and two-phase flow - see Supple- 
mentary Material for details. Numerical solution of the model provides the shape of the interface, as well as the capillary number for entrainment. The latter is shown in Fig. 4.3 (solid line). Since the model is derived by assuming small interface curvatures [29], it cannot be expected to be fully quantitative. Yet, it does capture the order of magnitude for $\mathrm{Ca}_{e}$ as well as the dependence on $\eta_{g} / \eta_{\ell}$.

In conclusion, we experimentally showed that the entrainment speed of advancing contact lines does not scale as $\gamma / \eta_{\ell}$, but exhibits a much weaker variation with liquid viscosity. We explain this by the influence of the air flow when the local angle of the interface is close to $\pi$. Can such a scenario explain the observed increase of entrainment speed when depressurizing the air $[23,65]$ ? A pressure reduction does not affect the dynamical viscosity of a gas [71]. However, as also mentioned in $[23,65]$, it does increase the mean free path $\ell_{\mathrm{mfp}}$ by a factor $\ell_{\mathrm{mfp}} \sim p_{\mathrm{atm}} / p$. Since under atmospheric conditions $\ell_{\mathrm{mfp}} \approx 70 \mathrm{~nm}$, the mean free path is pushed well into the micron range when pressure is reduced by a factor 100. The mean free path then becomes comparable to the film thickness measured experimentally. Since $\ell_{\mathrm{mfp}}$ sets the scale for the slip length [72], we expect a substantial reduction of dissipation in the gas, and hence a larger entrainment velocity. Indeed, upon introducing $\ell_{\mathrm{mfp}}$ as the slip length, the model yields an increase of $\mathrm{Ca}_{e}$ (Fig. 4.3, dotted line). This provides the exciting perspective that depressurized air is a Knudsen gas when entrained by advancing contact lines (Fig. 4.4c).

\subsection{Supplementary Material}

In this Supplementary Material, we describe the technical details of the experimental and theoretical results provided in this Chapter. First, we provide the two-phase lubrication equation, based on Huh \& Scriven's [6] analytical solution for the flow in a wedge, which was used to obtain the numerical results. Then, we derive an estimate for the viscous energy dissipation rates in both the liquid and gas phases. Third, we detail the experimental procedure used to measure the contact line velocities. Finally, the experimental procedure used to measure the film thickness is detailed.

\subsubsection{Two-phase hydrodynamic model}

The model summarized here will be presented in more detail in Chapter 5, where it will also be compared to lattice Boltzmann simulations. We consider a solid plate vertically translating across two immiscible fluids at a constant speed $U_{p}$. Below a threshold velocity $U_{e}$, the interface separating the two fluids deforms gradually and reaches stationary state. We investigate these stationary states of the interface 


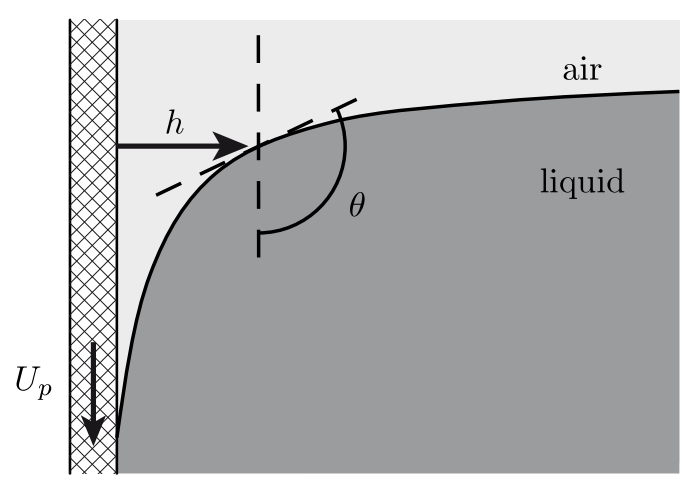

Figure 4.5: Schematic diagram of a plate plunging into a liquid reservoir with constant speed $U_{p}$.

and from this determine the critical velocity beyond which a film of one phase is entrained. The Reynolds number is assumed to be small such that the flow can be obtained from Stokes equation. We introduce the thickness $h$ of the film and the local interface angle $\theta$ as shown in Fig. 4.5. The origin of the vertical axis $x$ is taken at the contact line. Laplace's law relates the local curvature $\kappa$ of the interface to the normal stress difference across the interface $\tau_{g}$ and $\tau_{\ell}$ :

$$
\gamma \kappa=\gamma \frac{d \theta}{d s}=\tau_{\ell}-\tau_{g}
$$

where $\gamma$ is the surface tension between the two fluids and $s$ the curvilinear coordinate. $s=0$ is the contact line so that $h(s=0)=0$. Differentiating Eq. (4.1) with respect to $s$, we obtain

$$
\gamma \frac{d^{2} \theta}{d s^{2}}=\frac{d \tau_{\ell}}{d s}-\frac{d \tau_{g l}}{d s}
$$

To evaluate the derivatives of the normal stresses $\tau_{g}$ and $\tau_{\ell}$, we need to determine the velocity fields in both phases, which themselves depend on the shape of the interface. The exact resolution of this two-phase, multiscale, free boundary problem is extremely challenging. In the spirit of $[29,70]$, our approach here is to approximate the local velocity field by the flow field in the wedge of angle $\theta$, tangent to the curved interface [6]. Fig. 4.4a shows the corresponding streamlines of the flows in a wedge confining air in a very narrow region. For these solutions, the gradient of the nonisotropic part of the normal stress at the interface vanishes, such that normal stresses 
can be replaced by pressures. The equation governing the interface reads:

$$
\gamma \frac{d^{2} \theta}{d s^{2}}=\left[\eta_{g} \nabla^{2} \vec{U}_{g}-\eta_{\ell} \nabla^{2} \vec{U}_{\ell}-\vec{\nabla}\left(\Phi_{g}-\Phi_{\ell}\right)\right]_{\mathrm{int}} \cdot \vec{e}_{s}
$$

where $\vec{U}_{g}, \vec{U}_{\ell}, \eta_{g}, \eta_{\ell}, \Phi_{g}$ and $\Phi_{\ell}$ are the velocities, the viscosities and the gravitational potentials of gas and liquid respectively. $\vec{e}_{s}$ is the unit vector tangent to the interface. As indicated by the index "int", all quantities are evaluated at the interface. The viscous terms can be written as (using [6]):

$$
\eta_{\ell}\left[\frac{\eta_{g}}{\eta_{\ell}} \nabla^{2} \vec{U}_{g}-\nabla^{2} \vec{U}_{\ell}\right]_{i n t} \cdot \hat{e}_{s}=\frac{3 \eta_{\ell} U_{p} f\left(\theta, \frac{\eta_{g}}{\eta_{\ell}}\right)}{h^{2}},
$$

and

$$
\begin{aligned}
f(\theta, R) & \equiv \frac{2 \sin ^{3} \theta\left[R^{2} f_{1}(\theta)+2 R f_{3}(\theta)+f_{1}(\pi-\theta)\right]}{3\left[R f_{1}(\theta) f_{2}(\pi-\theta)-f_{1}(\pi-\theta) f_{2}(\theta)\right]} \\
f_{1}(\theta) & \equiv \theta^{2}-\sin ^{2} \theta \\
f_{2}(\theta) & \equiv \theta-\sin \theta \cos \theta \\
f_{3}(\theta) & \equiv\left(\theta(\pi-\theta)+\sin ^{2} \theta\right) .
\end{aligned}
$$

The gravity terms reads

$$
\left[\vec{\nabla}\left(\Phi_{\ell}-\Phi_{g}\right)\right]_{i n t} \cdot \hat{e}_{s}=-\left(\rho_{\ell}-\rho_{g}\right) g \cos \theta
$$

Finally, Eq. (4.3) takes the form:

$$
\frac{d^{2} \theta}{d s^{2}}=\frac{3 \mathrm{Ca}}{h^{2}} f\left(\theta, \frac{\eta_{g}}{\eta_{\ell}}\right)-\frac{\cos \theta}{\ell_{\gamma}^{2}}
$$

where

$$
\mathrm{Ca}=\frac{\eta_{\ell} U_{p}}{\gamma}, \quad \ell_{\gamma}=\sqrt{\frac{\gamma}{\left(\rho_{\ell}-\rho_{g}\right) g}} .
$$

This equation must be complemented by the geometrical relation

$$
\frac{d h}{d s}=\sin \theta
$$




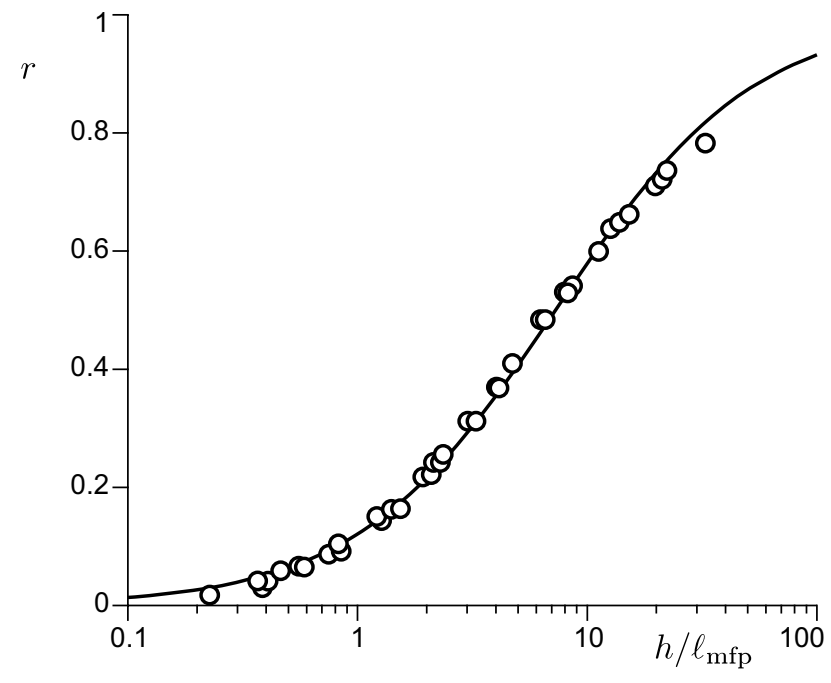

Figure 4.6: Correction factor $r$ that must be introduced in the viscous stress to describe a confined gas, as a function of Knudsen number defined as the ratio of thickness $h$ and mean free path $\ell_{\mathrm{mfp}}$. Data taken from [73], solid line is the best fit by (4.10).

In order to remove the singularity at the moving contact line, the liquid slip length $\lambda_{\ell}$ is introduced in the interface equation in a way such that it reduces to the standard lubrication equation as $\eta_{g} / \eta_{\ell} \rightarrow 0$ and $\theta \rightarrow 0$ [13]. For the air, it is known that the viscous friction gets reduced when confined in a film of thickness $h$ that is comparable to the mean free path $\ell_{\mathrm{mfp}}$ [73], since the number of collisions is reduced. Figure 4.6 shows experimental points of the correction factor $r$ that must be introduced to describe a confined gas obtained by Andrew \& Harris [73]. This graph shows that $r$ only depends on the Knudsen number, $h / \ell_{\mathrm{mfp}}$. The data are accurately fitted by

$$
r=\frac{h}{h+3 \lambda_{g}},
$$

suggesting that the air flow experiences an effective slip length $\lambda_{g}$. The best fit is obtained using $\lambda_{g}=2.4 \ell_{\mathrm{mfp}}$. Introducing the two slip lengths into our model, the interface equation becomes

$$
\frac{d^{2} \theta}{d s^{2}}=\frac{3 C a}{h\left(h+3 \lambda_{\ell}\right)} f\left(\theta, r \frac{\eta_{g}}{\eta_{\ell}}\right)-\cos \theta .
$$


Eq. (4.11) can be solved numerically to obtain the shape of the dynamical meniscus, similar to [13]. At the contact line, we assume that the microscopic contact angle $\theta(s=0)=\theta_{e}$ is independent of $\mathrm{Ca}$ (and equal to Young's contact angle). At the bath, the interface tends to a horizontal flat reservoir, i.e. $\theta(s \rightarrow \infty)=\pi / 2$. A typical solution is shown in Fig. 4.7. The maximum speed for static solutions is determined numerically for various $\eta_{g} / \eta_{\ell}$ and gives the curves in Fig. 4.3.

\subsubsection{Energy dissipation rate}

Here we derive the viscous energy dissipation rate in both liquid and air, based on the analytical solutions of a flow in a wedge due to Huh \& Scriven. We then evaluate the asymptotic behavior when the dynamic contact angle $\theta$ is close to $\pi$. We consider a circular region of radius $L$ and centered at the contact line. An inner cutoff length scale $\lambda$ (typically the slip length) is introduced to avoid the viscous singularity. The total viscous dissipation rate $\dot{E}_{v i s}$ inside the semicircular region of radius $L$ can be expressed as

$$
\dot{E}_{v i s}=2 \eta_{\ell} \int_{\lambda}^{L} \int_{0}^{\theta} e_{i j} e_{i j} r d \phi d r+2 \eta_{g} \int_{\lambda}^{L} \int_{\theta}^{\pi} e_{i j} e_{i j} r d \phi d r,
$$

where $e_{i j}$ is the rate-of-strain tensor. It is worth mentioning that the lubrication equation (4.7) can be recovered from the power balance equation. For this, one needs to balance the viscous dissipation computed above with the work done by capillary forces. These capillary forces consist of a contribution $\gamma\left(\cos \theta-\cos \theta_{e}\right)$ and the normal stress exerted on the boundaries of the control volume bounded by $\lambda$ and $L$.

For the wedge solution, the velocity field does not depend on $r$, so the components of $e_{i j}$ are of the form

$$
\begin{aligned}
e_{\phi \phi} & =\frac{1}{r}\left(\frac{\partial U_{\phi}}{\partial \phi}+U_{r}\right)=0, \\
e_{r r} & =0 \\
e_{r \phi} & =\frac{r}{2} \frac{\partial}{\partial r}\left(\frac{U_{\phi}}{r}\right)+\frac{1}{2 r} \frac{\partial U_{r}}{\partial \phi} .
\end{aligned}
$$

Evaluating the flow field of [6], we get:

$$
\dot{E}_{v i s}=-3 \eta_{\ell} U^{2} \ln \left(\frac{L}{\lambda}\right) \frac{f(\theta, R)}{\sin \theta} .
$$

Since air entrainment occurs when $\theta$ is close to $\pi$, we study the asymptotic behavior of $\dot{E}_{v i s}$ in this limiting case. When $\pi-\theta$ is still much larger than $\left(\eta_{g} / \eta_{\ell}\right)^{1 / 3}, \dot{E}_{v i s}$ 

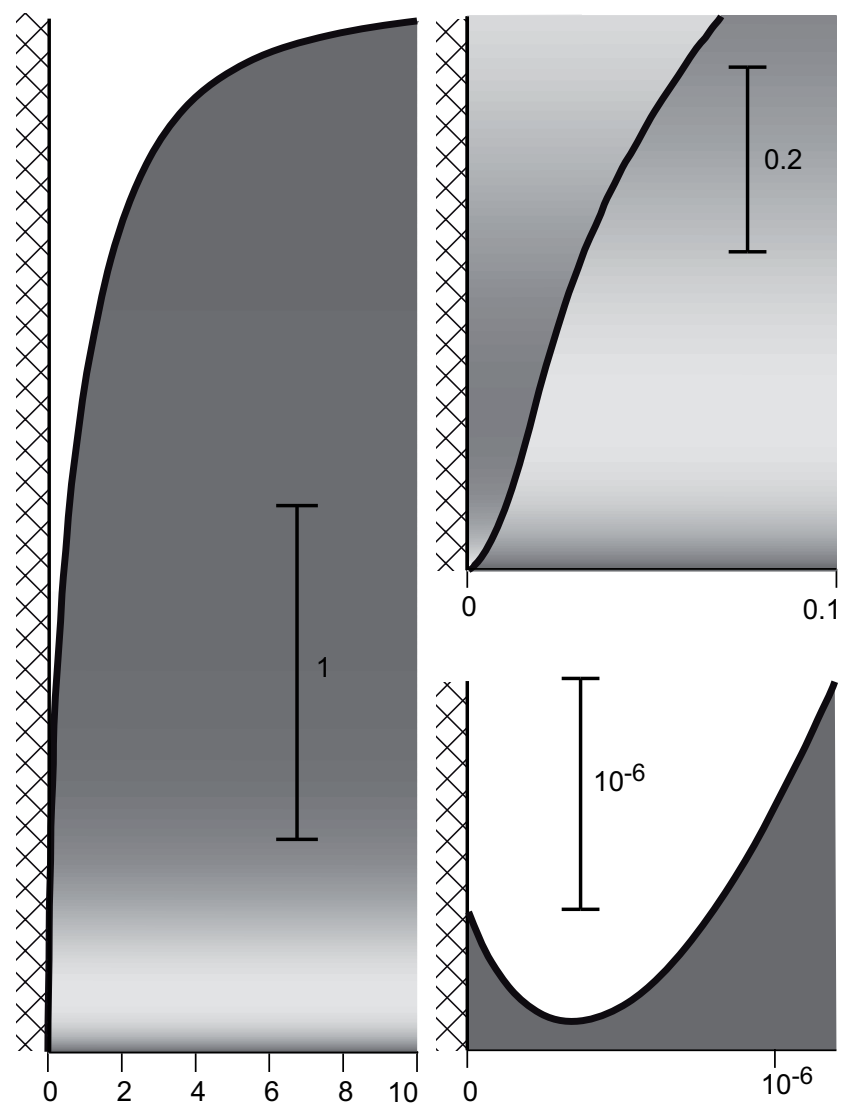

Figure 4.7: Shape of the air/liquid interface predicted by the hydrodynamic model (4.11). Just below the entrainment threshold, the meniscus, seen at different scales on the three panels, is stationary and connects the contact line to the bath. The graylevel indicates the fraction of the total dissipation due to each phase; the relative importance of gas and liquid depends on the scale. Axis are in units of capillary length $\ell_{\gamma}$.

goes as

$$
\dot{E}_{v i s}=3 \eta_{\ell} U^{2} \ln \left(\frac{L}{\lambda}\right)(\pi-\theta)^{2} .
$$

In this case the liquid still dominates. But once $\pi-\theta$ becomes much smaller than 
$\left(\eta_{g} / \eta_{\ell}\right)^{1 / 3}, \dot{E}_{v i s}$ goes as

$$
\dot{E}_{v i s}=\frac{12 \eta_{g} U^{2} \ln \left(\frac{L}{\lambda}\right)}{\pi-\theta} .
$$

When the film is sufficiently flat, the air becomes dominant even though the air viscosity $\eta_{g}$ is much smaller than the liquid viscosity $\eta_{\ell}$.

\subsubsection{Measurements of the contact line velocities}

Fig. 4.8 shows a space-time diagram obtained in the central part of the silicon wafer (see Fig. 4.1c). The velocity of both the plate $U_{p}$ and the contact line $U_{c l}$ can be determined in the frame of reference of the laboratory, by measuring the slopes of the lines appearing in the space-time diagram.

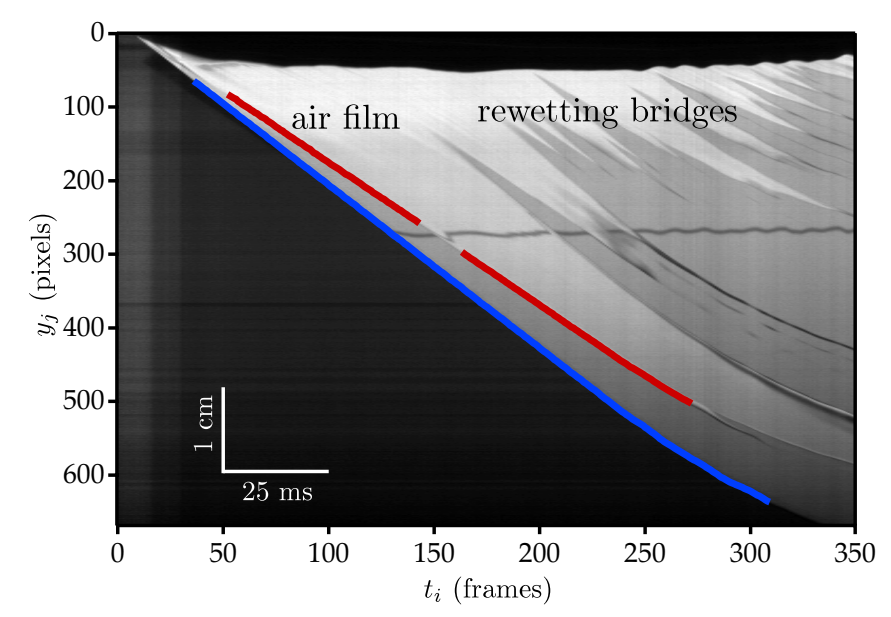

Figure 4.8: Space-time diagram obtained by extracting a vertical line from a high speed movie (2000 frames per second). Here the plate plunges at $50 \mathrm{~cm} / \mathrm{s}$ into a liquid bath of viscosity $\eta_{\ell}=0.5$ Pa.s. The wafer trajectory is detected in blue with an algorithm based on a maximum correlation. The contact line, shown in red, is obtained with the same algorithm - the air film corresponds to the lighter areas. Note the triangular shapes corresponding to rewetting bridges. Note also the black horizontal line, which is the image of a straight wire through the wafer, which is distorted by the interface and therefore gives the slope of the interface profile.

These velocity measurements are based on a correlation technique. We first determine the position of the contact line by looking at the transition between light and 
dark areas. We determine the maximum of the grey level gradient (using a 10 pixels kernel) with a sub-pixel resolution. We then measure the contact line velocity by maximizing the image correlation with respect to the slope, again with a sub-pixel resolution. The entrainment velocity is finally deduced in this first method from the difference between the plate velocity and the contact line velocity in the laboratory frame.

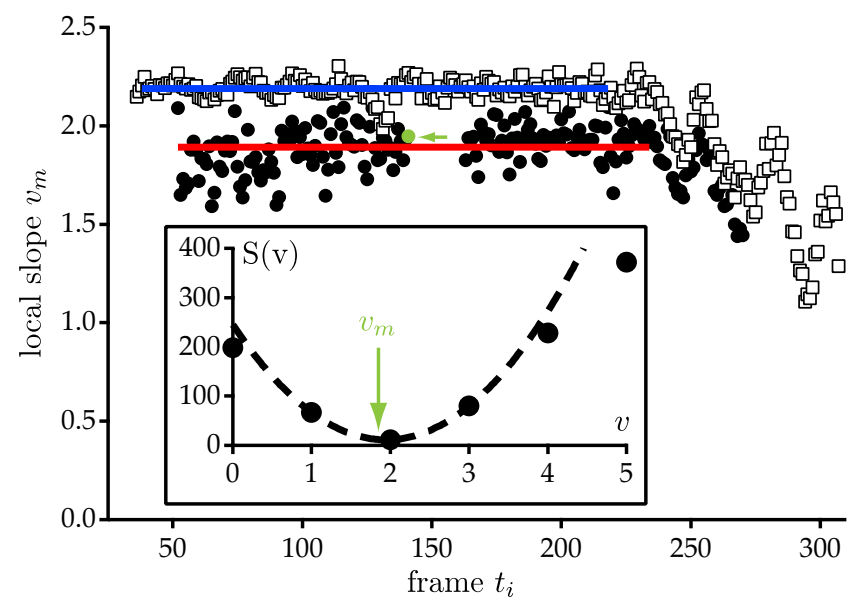

Figure 4.9: Velocity measurement of the contact line and of the wafer. Inset: Correlation $S(v)$ between three consecutive frames of the sequence shown in Figure 4.8, once translated at the velocity $v$. The minimum represents the local slope, and is detected at the position of the green arrow by fitting data to a parabola (dotted line). The minimum is almost at zero, which indicates that the correlation is good. Main graph: local velocity along the detected lines of Figure 4.8 . The mean values are represented by the blue and red lines and are measured before the breaking phase at $t_{i} \approx 250$. The green point represents the value measured in panel (a). The wafer velocity is finally measured here at $U_{p}=44.7 \mathrm{~cm} / \mathrm{s}$ and the contact line velocity at $U_{c l}=38.6 \mathrm{~cm} / \mathrm{s}$.

For high viscosities, the rewetting bridges destabilize rapidly the contact line, and the direct velocity measurement is less precise. However, one can measure with the same tools the growth velocity of the rewetting bridges, in their frame of reference. We observe that both vertical and horizontal velocities are the same in the hole frame, as the holes remain almost always circular. 


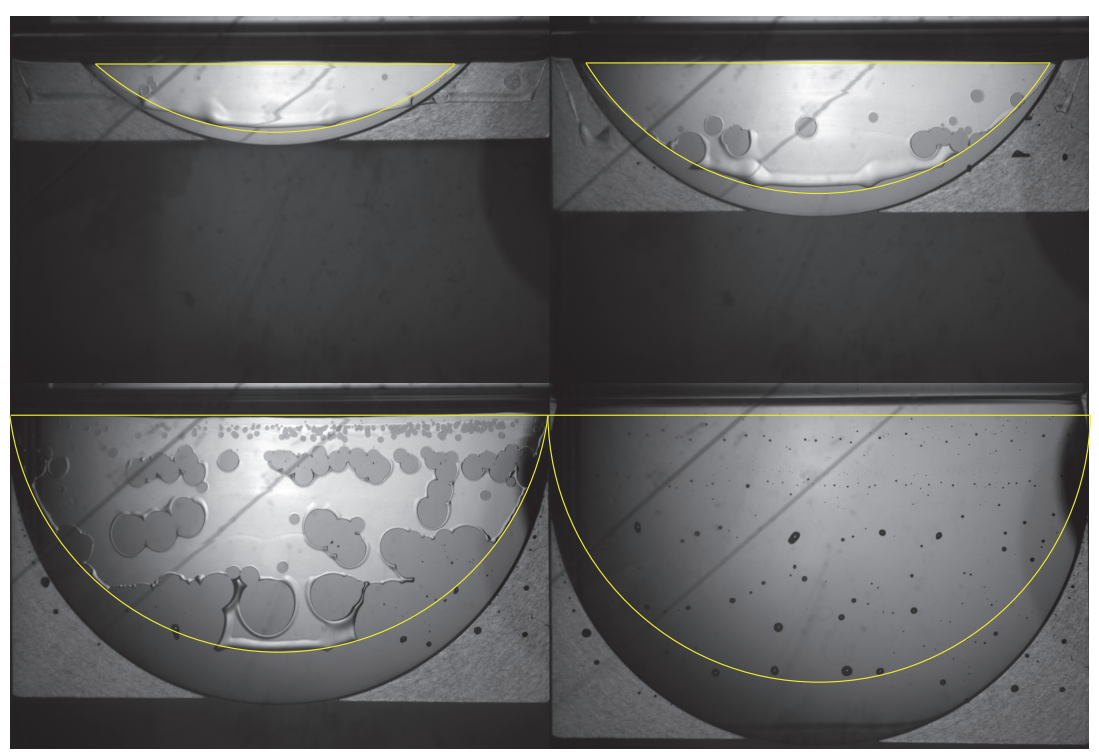

Figure 4.10: Four images showing the development of the air film and the formation of spherical bubbles that slowly rise to the surface. The yellow contours show our estimate of the film surface at each time. The film thickness is determined as the ratio of the bubble volume (determined accurately from the last image) to the film surface (shown in yellow in the last image).

\subsubsection{Measurements of the film thickness}

The air film thickness is determined as the volume of air entrapped in the liquid divided by the surface of the film. More precisely, we consider the surface of film produced close to the bath. When rewetting bridges appear, we still consider the surface that would have been covered if the film had been stable. This is illustrated in Fig. 4.10 where one can see the appearance of a flat film (top left), then the appearance of the first rewetting bridges, and finally the progressive destruction of the contact line. The effective surface of air taken into account is the surface enclosed by the yellow line. The upper part corresponds to the level at which the film starts, few millimeters below the bath level. The lower part is a portion of circle of the same diameter as the wafer, moved downward at the mean contact line velocity.

The air bubbles trapped at the end of an experiment are imaged with a camera Nikon D300s (4288x2848 pixels) mounted with macro-lens. The size of each bubble is determined within a $10 \mu \mathrm{m}$ resolution, by fitting an elliptic shape. The 
bubbles slowly rise at constant velocity along the wafer. We wait for the relaxation of their shapes to almost perfect spheres before performing the measurement. We have checked on few examples that their rising velocity was consistent with the geometric determination of their radius. Looking from the side, we have checked that there is not a single bubble attached to the wafer.

The measurement of the volume of air entrained turned out to be both very reproducible and very accurate. Most of the uncertainty on $h$ actually results from the estimate of the surface covered by the air film (yellow line in Fig. 4.10). We have performed each experiment several times, with an independent determination of the bubble and of the surface. The statistical error on the volume is very low while that on the surface depends a lot on the viscosity. When the film remains stable for a long time, we get a correct reproducibility but this becomes harder and harder to achieve when the film gets more fragile. Statistical error bars therefore reflects adequately the difficulty encountered in the measurement. 


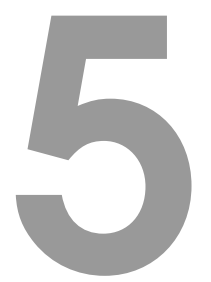

\section{Hydrodynamics of air entrainment by moving contact lines $* \dagger$}

\subsection{Introduction}

The preceding Chapter revealed a surprising scenario for air entrainment by rapidly advancing contact lines. By plunging a plate into reservoirs of different liquids, we observed an unexpectedly weak dependence of the critical speed for air entrainment on the liquid viscosity $\eta_{\ell}$. The perturbation analysis by Cox [21] suggested that the critical speed scales as $1 / \eta_{\ell}$, with logarithmic corrections due to the viscosity of the air. This is similar to the scenario for air entrainment by viscous cusps [20], such as observed for impacting liquid jets [19]. Our experiments for advancing contact lines, however, show that the dependence on $\eta_{\ell}$ is much weaker than predicted; enforcing a power-law fit to our data would give a small exponent, in between $-1 / 2$ and $-1 / 3$ rather than the expected -1 . In the preceding Chapter we argued that this can be attributed to the viscous stress inside the air, which is strongly enhanced when the apparent contact angle approaches $\pi$. The importance of air was already highlighted in similar dip-coating experiments, where a reduction of the ambient pressure

\footnotetext{
${ }^{*}$ The part related to the generalized lubrication model in this chapter is part of the present thesis. The lattice Boltzmann simulations were performed by Sudhir Srivastava.

${ }^{\dagger}$ Manuscript to be submitted: T.S. Chan, S. Srivastava, A. Marchand, B. Andreotti, L. Biferale, F. Toschi and J.H. Snoeijer, Hydrodynamics of air entrainment by moving contact lines.
} 
was shown to significantly enhance the critical speed of entrainment [23]. Yet, this raises another paradox: the dynamical viscosity of the air is virtually unchanged by a pressure reduction.

In this Chapter we provide a new framework to study air entrainment by advancing contact lines, in which the two-phase character of the flow is taken into account. The usual lubrication approximation is valid for small (liquid) contact angles and does not take into account the flow inside the gas. Since both assumptions are no longer valid near the onset of air entrainment, we extend the lubrication approximation such that it allows for large angles and a nonzero gas viscosity. This approach is validated by comparing to Lattice Boltzmann simulations. We show that the meniscus shapes obtained from the generalized lubrication model agrees well with the simulations. Note, however, that such simulations are limited in terms of viscosity ratio and spatial resolution (i.e. the separation between the capillary length and the microscopic cutoff), and cannot achieve experimental conditions for air entrainment. To compare to experiments and to explore the parameter space, in particular the importance of air viscosity $\eta_{g}$, we therefore provide a detailed study using the generalized lubrication model.

\subsection{Formulation}

We consider a smooth, chemically homogeneous solid plate translating across an interface of two immiscible fluids at a constant speed $U_{p}$ (positive/negative for plunging/withdrawing). As sketched in Fig. 5.1, the two liquids are contained in a reservoir much larger than all the lengths of the problem and the plate can be inclined to any angle $\alpha$. If the plate is not moving $\left(U_{p}=0\right)$ there is no flow in the fluids, and the interface equilibrates to a static shape due to balance between capillarity and gravity. The interface makes an equilibrium angle $\theta_{e}$ with the solid as a result of intermolecular interaction between the three phases at the contact line. Neglecting the contact angle hysteresis, $\theta_{e}$ takes a well-defined value determined by Young's law. The contact line equilibrates at a distance $\Delta$ above the bath, which can be expressed as

$$
\Delta= \pm \ell_{\gamma} \sqrt{2\left[1-\cos \left(\alpha-\theta_{e}\right)\right]} .
$$

Here, $\ell_{\gamma}=\sqrt{\frac{\gamma}{\left(\rho_{\ell}-\rho_{g}\right) g}}$ is the capillary length, defined by surface tension $\gamma$, gravity $g$ and the density difference $\rho_{\ell}-\rho_{g}$. The \pm sign depends on whether $\theta_{e}$ is smaller $(+)$ or larger (-) than the plate inclination $\alpha$. When addressing the transition to air entrainment, we will consider the upper phase A in Fig. 5.1 to be gaseous, while phase $\mathrm{B}$ is a liquid. We therefore use subscripts ' $\ell$ ' and ' $g$ ' to indicate liquid and gas 


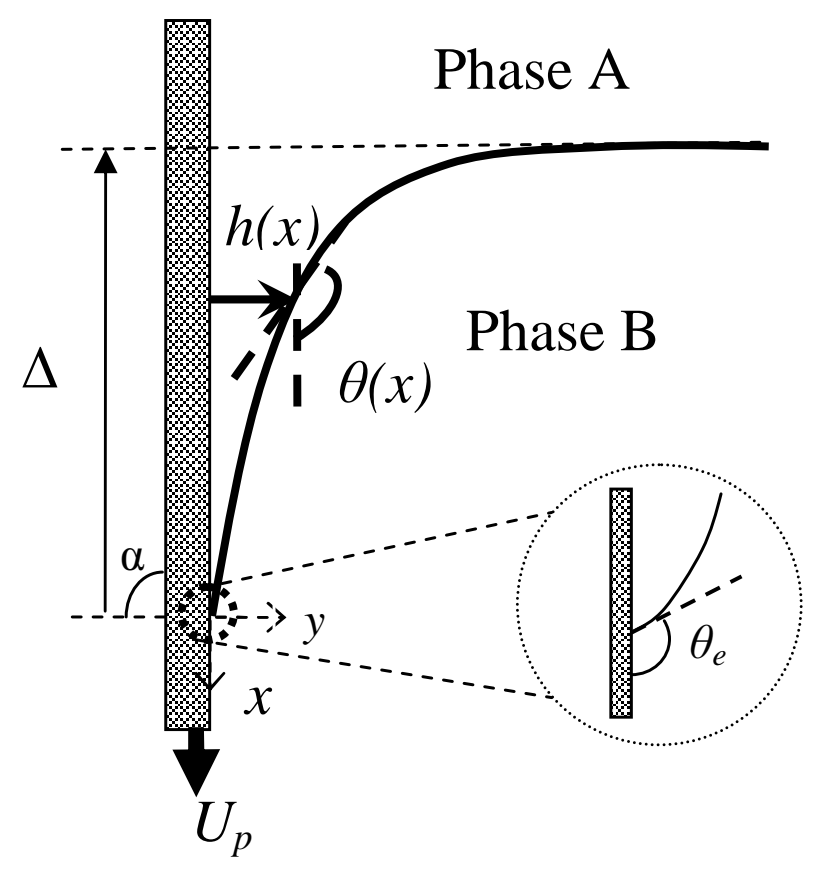

Figure 5.1: Schematic diagram showing a plate moving with speed $U_{p}$ with respect to an interface of two immiscible fluids. The plate has an inclination $\alpha$, here drawn with $\alpha=\pi / 2$ for the case of a vertical plate. The interface touches the wall at the contact line with an angle assumed to be the same as the equilibrium contact angle $\theta_{e}$. The meniscus profile is described by $h(x)$ or the local angle $\theta(s)$, where $s$ is the arc length of the interface measured from the contact line. The total meniscus deformation is quantified by $\Delta$, the distance between the contact line and the level of the bath. 
phase respectively.

When the plate is moving, the viscous drag generated by the moving plate gradually deforms the fluid-fluid interface. As long as the speed of the plate is below a threshold value, the meniscus equilibrates at a new value of $\Delta$ (see Fig. 5.1). For positive $U_{p}$ the plate is moving downwards and $\Delta$ will be below the static equilibrium height, while the opposite holds for negative $U_{p}$. The contact line is assumed to be straight so that the problem can be treated as two-dimensional. The interface profile is described by film thickness $h(x)$. The contact line position is at $x=0$. When the plate is moving beyond a critical speed, the meniscus can no longer equilibrate to a steady state. In the case of receding contact lines (withdrawing plate) this leads to the deposition of a liquid film [3, 12-14, 18, 74, 75], while air will be entrained for advancing contact lines $[2,23,39,62-65,76]$.

In this study we only focus on viscous flows, for which the Reynolds number is assumed to be zero. Thus for incompressible fluids, the flow fields in the fluids are described by Stokes equations and continuity,

$$
\eta_{g} \nabla^{2} \vec{u}_{g}-\vec{\nabla} p_{g}-\vec{\nabla} \Phi_{g}=0, \quad \vec{\nabla} \cdot \vec{u}_{g}=0
$$

and

$$
\eta_{\ell} \nabla^{2} \vec{u}_{\ell}-\vec{\nabla} p_{\ell}-\vec{\nabla} \Phi_{\ell}=0, \quad \vec{\nabla} \cdot \vec{u}_{\ell}=0,
$$

where $\vec{u}_{g}$ and $\vec{u}_{\ell}, p_{g}$ and $p_{\ell}, \Phi_{g}$ and $\Phi_{\ell}$ are the corresponding velocity fields, pressures and gravitational potentials in phase $\mathrm{A}$ and phase $\mathrm{B}$ respectively. When considering air entrainment, phase $\mathrm{A}$ is assumed a gas with viscosity $\eta_{g}$, and phase B is liquid with viscosity $\eta_{\ell}$. The relative viscosity is expressed by the viscosity ratio $R=\eta_{g} / \eta_{\ell}$ which, in practical situations, can be very small.

To solve for the flow fields and the interface shape, we need to specify appropriate boundary conditions. At the steady interface, the velocities parallel to the interface $u^{t}$ are continuous and the velocities normal to the interface $u^{n}$ vanish, so

$$
u_{g}^{t}=u_{\ell}^{t}
$$

and

$$
u_{g}^{n}=u_{\ell}^{n}=0 .
$$

In each phase, we define the normal stresses $\sigma^{n} \equiv \hat{n} \cdot \hat{\sigma} \cdot \hat{n}$ at the interface, where $\hat{n}$ is the unit vector normal to the interface and the stress tensor $\widehat{\sigma}$ is defined as (in Cartesian coordinates)

$$
\sigma_{i j} \equiv-p \delta_{i j}+\eta\left(\frac{\partial u_{i}}{\partial x_{j}}+\frac{\partial u_{j}}{\partial x_{i}}\right)
$$


The normal stress discontinuity across the interface is related to the curvature $\kappa$ and to the surface tension $\gamma$ by Laplace's law

$$
\sigma_{\ell}^{n}-\sigma_{g}^{n}=\gamma \kappa
$$

By contrast, the stresses parallel to the interface $\sigma^{t} \equiv \hat{t} \cdot \hat{\sigma} \cdot \hat{n}$ are continuous at the interface,

$$
\sigma_{g}^{t}=\sigma_{\ell}^{t}
$$

At the solid/fluid boundary $(y=0)$, the velocity normal to the wall $u_{y}(y=0)$ vanishes as no penetration of fluid through the solid is allowed, i.e.

$$
u_{y}(y=0)=0 .
$$

Regarding the velocity component parallel to the wall, $u_{x}(y=0)$, the situation is more subtle because of the moving contact line singularity $[6,9]$. Namely, imposing a no-slip boundary condition leads to diverging stress fields and calls for a microscopic mechanism of regularization. In the following section we present two methods to solve the flow equations, a generalized lubrication model and Lattice Boltzmann simulations, which naturally involve different regularizations of the singularity. The microscopic boundary condition will therefore be discussed separately below.

\subsection{Methods}

In this section we present two methods to determine the meniscus shape sketched in Fig. 5.1. We first present a model that can be considered as a generalization of the standard lubrication approximation. We then present the Lattice Boltzmann method, which is a rather different approach to solve for the flow and the meniscus shape. The models will be referred to as GL (Generalized Lubrication model) and LB (Lattice Boltzmann).

\subsubsection{Generalized Lubrication model}

\section{Derivation}

The lubrication approximation has been a very efficient framework to deal with thin film flows [10]. This systematic reduction of the Navier-Stokes equations is very suitable for numerical simulations and in many cases allows for analytical results [9]. It is usually derived for flows that involve a single phase that constitutes a "thin" film, i.e. 
the slopes $d h / x$ are assumed small. However, the expansion parameter underlying the analysis is not the interface slope, but the capillary number $\mathrm{Ca}=U \eta_{\ell} / \gamma$ [10]. This means that a lubrication-type analysis can be performed whenever surface tension dominates over viscosity. Indeed, it was shown in [29] that the lubrication approximation can be generalized to large interface angles $\theta$, giving a perfect agreement with the perturbation results by Voinov [27] and Cox [21]. Here we further generalize this approach, by including not only the effect of a large slope, but also taking into account the viscous flows at both sides of the interface. The goal is to provide a model that can deal with moving contact lines in cases where both phases are important (as in Fig. 5.1). In particular, this will allow studying the air entrainment transition.

Let us now derive this generalized lubrication model (GL). As mentioned in the preceding section, the interface curvature $\kappa$ is determined by the normal stresses difference across the interface. In curvilinear coordinates, we write

$$
\gamma \kappa \equiv \gamma \frac{d \theta}{d s}=\sigma_{\ell}^{n}-\sigma_{g}^{n}
$$

where $\theta$ is the local tangential angle and $s$ the arc length of the interface (see Fig. 5.1). The normal stresses have to be determined from the flows in the fluids, which themselves depend on the shape of the interface. For the usual lubrication theory in which the interface slope is small, the leading order contribution of the flow reduces to a parabolic Poiseuille flow inside the film. This can be generalized to two-phase flows and large interface slopes: as long as the capillary number is small, the interface curvature will be small and the leading order velocity field is given by the flow in a wedge (Fig. 5.2). The wedge solutions were obtained analytically already by Huh \& Scriven, and can be solved for any viscosity ratio $R=\eta_{g} / \eta_{\ell}$ and for any wedge angle $\theta$. Fig. 5.2 shows the corresponding streamlines. Based on these exact solutions, the local normal stresses can be determined, thus giving the local curvature of the interface through Eq. (5.10).

We denote the quantities derived from Huh-Scriven solution by capital symbols, e.g. normal stress is denoted as $\Sigma^{n}$, velocity as $\vec{U}$ and pressure as $P$. For the HuhScriven solution, it turns out that the non-isotropic part of the normal stress at the interface vanishes so that

$$
\Sigma^{n}=-P
$$

Approximating the normal stresses by the Huh-Scriven solutions, Eq. (5.10) becomes

$$
\gamma \frac{d \theta}{d s}=\Sigma_{\ell}^{n}-\Sigma_{g}^{n}=P_{g}-P_{\ell}
$$


Since $P_{g}-P_{\ell}$ are defined up to an integration constant, it is convenient to differentiate Eq. (5.12) once with respect to $s$, giving

$$
\gamma \frac{d^{2} \theta}{d s^{2}}=\frac{d P_{g}}{d s}-\frac{d P_{\ell}}{d s}=\left[\vec{\nabla} P_{g}-\vec{\nabla} P_{\ell}\right]_{i n t} \cdot \hat{e}_{s} .
$$

The index "int" indicates that the quantities inside the brackets are to be evaluated on the interface and $\hat{e}_{s}=\hat{t}$ is the unit vector tangent to the interface.

When Stokes equation (5.2) is applied, Eq. (5.13) can be rephrased in terms of the Huh-Scriven velocity fields, i.e.

$$
\gamma \frac{d^{2} \theta}{d s^{2}}=\left[\eta_{g} \nabla^{2} \vec{U}_{g}-\eta_{\ell} \nabla^{2} \vec{U}_{\ell}-\vec{\nabla}\left(\Phi_{g}-\Phi_{\ell}\right)\right]_{i n t} \cdot \hat{e}_{s} .
$$

The viscous contributions on the right hand side can be expressed in terms of $R$ and $\theta$ in the form

$$
\eta_{\ell}\left[\frac{\eta_{g}}{\eta_{\ell}} \nabla^{2} \vec{U}_{g}-\nabla^{2} \vec{U}_{\ell}\right]_{i n t} \cdot \hat{e}_{s}=\frac{3 \eta_{\ell} U_{p} f(\theta, R)}{h^{2}}
$$

where

$$
\begin{aligned}
f(\theta, R) & \equiv \frac{2 \sin ^{3} \theta\left[R^{2} f_{1}(\theta)+2 R f_{3}(\theta)+f_{1}(\pi-\theta)\right]}{3\left[R f_{1}(\theta) f_{2}(\pi-\theta)-f_{1}(\pi-\theta) f_{2}(\theta)\right]} \\
f_{1}(\theta) & \equiv \theta^{2}-\sin ^{2} \theta \\
f_{2}(\theta) & \equiv \theta-\sin \theta \cos \theta \\
f_{3}(\theta) & \equiv\left(\theta(\pi-\theta)+\sin ^{2} \theta\right) .
\end{aligned}
$$

The gravity terms in (5.14) can be simplified to

$$
\vec{\nabla}\left[\Phi_{g}-\Phi_{\ell}\right]_{i n t} \cdot \hat{e}_{s}=-\left(\rho_{\ell}-\rho_{g}\right) g \sin (\theta-\alpha),
$$

where $\rho_{g}$ and $\rho_{\ell}$ are the densities of the two phases.

The final result of the analysis is a generalized form of the lubrication equation, which after scaling all lengths with the capillary length $l_{\gamma}=\sqrt{\frac{\gamma}{\left(\rho_{\ell}-\rho_{g}\right) g}}$ becomes

$$
\frac{d^{2} \theta}{d s^{2}}=\frac{3 \mathrm{Ca}}{h^{2}} f(\theta, R)+\sin (\theta-\alpha) .
$$

In the remainder we will use the same symbols for rescaled lengths. Once more, the capillary number is defined based on the viscosity of the liquid, $\mathrm{Ca}=\eta_{\ell} U_{p} / \gamma$. This equation must be complemented by the geometrical relation 


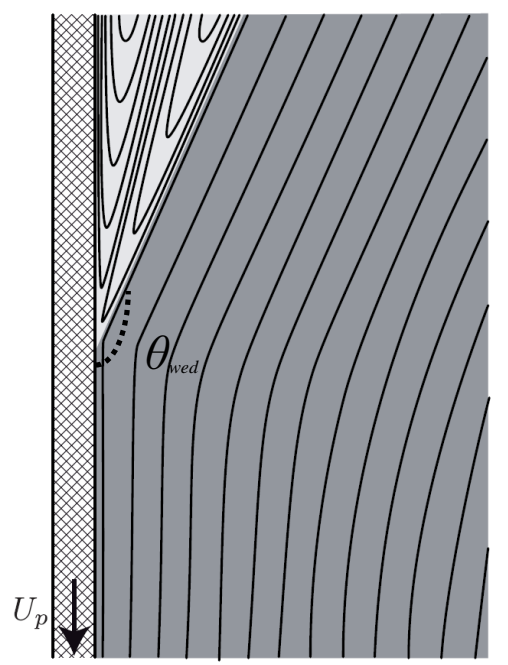

Figure 5.2: Streamlines of flow in a wedge of angle $\theta_{\text {wed }}$, which in this case is close to $\pi$. This flow solution was derived analytically by Huh \& Scriven [6], and is used here to derive a generalized lubrication model.

$$
\frac{d h}{d s}=\sin \theta
$$

Note that for all numerical examples in the remainder of this Chapter we consider a vertical plate, for which $\alpha=\pi / 2$.

One easily verifies that the standard lubrication equation is recovered when taking the limit of vanishing $R, \theta$ and $\alpha$. Namely, $f(0,0)=-1$ and (5.18) reduces to

$$
h^{\prime \prime \prime}=\frac{-3 \mathrm{Ca}}{h^{2}}+h^{\prime}-\alpha .
$$

Remember that $\mathrm{Ca}$ is negative when the plate is moving upward. When considering a single phase with arbitrary slope, one recovers the equation previously proposed in [29], with $f(\theta, 0)$ instead of $f(\theta, R)$.

\section{Slip boundary condition}

It is important to note that Eq. (5.18) is derived from the Huh-Scriven solution with no-slip boundary condition. Near the contact line, however, this induces a divergence of the pressure and of the shear stress, which scale as $\sim \eta_{\ell} U_{p} / h$ and $\sim \eta_{g} U_{p} / h$ in 
the liquid and the gas respectively. A purely hydrodynamic approach to regularize the singularity is to impose a slip boundary condition on the solid wall. No solution for the flow in a wedge with slip can be obtained analytically. However, as contact line flows are only mildly affected by the details of the microscopic conditions [9, 77], we proceed by a phenomenological regularization. We therefore consider the standard lubrication equation, which can be derived including a Navier slip boundary condition. It reads

$$
h^{\prime \prime \prime}=\frac{3 \mathrm{Ca}}{h\left(h+3 \lambda_{s}\right)}-h^{\prime}+\alpha,
$$

where $\lambda_{s}$ is the slip length. In comparison to (5.20), the effect of slip can be summarized by a correction factor $h /\left(h+3 \lambda_{s}\right)$ for the viscous term. Indeed, this weakens the singularity such that the equations can be integrated to $h=0$ [78].

We simply propose to use the same regularization factor for the viscous term in (5.18), i.e.

$$
\frac{d^{2} \theta}{d s^{2}}=\frac{3 \mathrm{Ca}}{h\left(h+3 \lambda_{s}\right)} f(\theta, R)+\sin (\theta-\alpha) .
$$

The appropriate boundary conditions are that the equilibrium contact angle $\theta_{e}$ is recovered at the contact line, and that the interface attains the angle of the reservoir at infinity:

$$
h(s=0)=0, \quad \theta(s=0)=\theta_{e} \quad \text { and } \quad \theta(s \rightarrow \infty)=\alpha .
$$

The meniscus shape is now fully determined by the lubrication equation (5.22), geometry (5.19) and boundary conditions (5.23). For a given value of the capillary number $\mathrm{Ca}$, the model parameters are the viscosity ratio $R$, the contact angle $\theta_{e}$, and the microscopic length $\lambda_{s}$. Below we compute the shape of meniscus for different parameter by numerical solution, using a 4 th order Runge-Kutta numerical scheme. As the boundary conditions are imposed at different locations, the solution is determined using a shooting algorithm.

\subsubsection{Lattice Boltzmann Method}

In this section we discuss some of the key features of lattice Boltzmann method (LBM). In a LBM model, the following discrete Boltzmann equation is solved for the single particle distribution function $f_{i, \alpha}(\mathbf{x}, t)$ over a $2 \mathrm{D}$ square lattice:

$$
f_{i, \alpha}\left(\mathbf{x}+\mathbf{e}_{i} \Delta t, t+\Delta t\right)-f_{i, \alpha}(\mathbf{x}, t)=\Omega_{i, \alpha}(\mathbf{x}, t)
$$


where

$$
\Omega_{i, \alpha}(\mathbf{x}, t)=-\frac{1}{\sigma_{\alpha}}\left[f_{i, \alpha}(\mathbf{x}, t)-f_{i, \alpha}^{\mathrm{eq}}(\mathbf{x}, t)\right],
$$

is the single time relaxation, linear BGK collision operator [79], $f_{i, \alpha}^{\mathrm{eq}}(\mathbf{x}, t)$ is the discrete Maxwell distribution function defined as:

$$
f_{i, \alpha}^{\mathrm{eq}}(\mathbf{x}, t)=\rho_{\alpha} w_{i}\left[1+\frac{\mathbf{e}_{i} \cdot \mathbf{u}_{\alpha}}{c_{s}^{2}}+\frac{\mathbf{e}_{i} \cdot \mathbf{u}_{\alpha}}{2 c_{s}^{4}}-\frac{\mathbf{u}_{\alpha} \cdot \mathbf{u}_{\alpha}}{2 c_{s}^{2}}\right]
$$

where

$$
\rho_{\alpha}=\sum_{i} f_{i, \alpha}, \quad \rho_{\alpha} \mathbf{u}_{\alpha}=\sum_{i} f_{i, \alpha} \mathbf{e}_{i}
$$

and $w_{i}, \mathbf{e}_{i}$ in (5.25) are the weights and the corresponding lattice speeds respectively $[80,81]$. The weights, $w_{i}$, corresponding to the 2-dimensional and 9 velocity model, D2Q9, are given by $w_{0}=4 / 9, w_{1}=w_{2}=w_{3}=w_{4}=1 / 9$, and $w_{5}=w_{6}=w_{7}=w_{8}=$ $1 / 36$. The total fluid density is $\rho=\sum_{\alpha} \rho_{\alpha}$ and the total hydrodynamic velocity is $\mathbf{u}=$ $\sum_{\alpha} \rho_{\alpha} \mathbf{u}_{\alpha} / \rho$. The effective kinematic viscosity is related to the relaxation time of the different components $v=\sum_{\alpha} c_{s}^{2}\left(\sigma_{\alpha} c_{\alpha}-0.5\right)$ [82], $c_{\alpha}=\rho_{\alpha} / \rho$ is the concentration, and $c_{s}=1 / \sqrt{3}$ is the speed of sound on the lattice. In absence of an external force, each component satisfies the ideal gas equation of state $p=c_{s}^{2} \rho$. For multicomponent simulation we are using two distribution functions $(\alpha=1,2)$, whereas for multiphase simulations we restrict ourselves to only one distribution function $(\alpha=1)$.

\section{Multiphase/multicomponent model}

The multicomponent/multiphase algorithm is based on a standard Shan-Chen lattice Boltzmann method [82-84]. The non-ideal nature of the fluid is introduced by adding an internal force by shifting the lattice Boltzmann equilibrium velocity as

$$
\mathbf{u}_{\alpha}^{\mathrm{eq}}=\mathbf{u}^{\prime}+\frac{\sigma_{\alpha} \mathbf{F}^{\alpha}}{\rho_{\alpha}}, \text { where } \mathbf{u}^{\prime}=\frac{\sum_{\alpha} \rho_{\alpha} \mathbf{u}_{\alpha} / \sigma_{\alpha}}{\sum_{\alpha} \rho_{\alpha} / \sigma_{\alpha}} .
$$

For the non-ideal interaction the force $\mathbf{F}_{\alpha}$ in the Shan-Chen model [82, 84] is given by:

$$
\mathbf{F}_{\alpha}=-G_{\alpha \alpha^{\prime}} \psi_{\alpha}(\mathbf{x}) \sum_{i, \alpha \neq \alpha^{\prime}} w_{i} \psi_{\alpha^{\prime}}\left(\mathbf{x}+\mathbf{e}_{i}\right) \mathbf{e}_{i}
$$

where $\left\{\alpha, \alpha^{\prime}\right\}=\{1,2\}$ are indices for two fluid components while the coupling parameter $G_{\alpha \alpha^{\prime}}$ is the strength of the interaction and determines the surface tension 
in the model. This force allows the formation of interface between the different fluid components. For multicomponent simulations $G_{12}=G_{21}=G, G_{11}=G_{22}=0$, $\psi_{\alpha}=\rho_{\alpha}$. In the case of multiphase simulations $\alpha=1, \psi=1-\exp (-\rho)$. The equation of state is modified to $p=c_{s}^{2}\left(\rho_{1}+\rho_{2}\right)+G c_{s}^{2} \rho_{1} \rho_{2}$ and $p=c_{s}^{2} \rho+\frac{G}{2} c_{s}^{2} \psi^{2}$ respectively for multicomponent and multiphase simulations, where the first term correspond to the ideal gas and the second term is the non-ideal part due to the external Shan-Chen force.

\section{Boundary conditions for LBM simulations}

The no-slip boundary condition for the fluid on corresponds to the bounce back boundary condition [80] for the distribution functions $f_{i, \alpha}(\mathbf{x}, \mathbf{t})$ defined at the boundary nodes.

The surface wetting for multicomponent simulations is introduced by adding an additional force at the wall $[85,86]$

$$
\mathbf{F}_{\alpha}^{a d s}=-G_{\alpha}^{a d s} \rho_{\alpha}(\mathbf{x}, t) \sum_{i} w_{i} s\left(\mathbf{x}+\mathbf{e}_{i}\right) \mathbf{e}_{i}
$$

where, $s\left(\mathbf{x}+\mathbf{e}_{i}\right)=1$ for a wall node and is 0 for a fluid node. The parameter $G_{\alpha}^{a d s}$ can be varied to control the wetting properties of the wall; in all our simulations we have used $G_{1}^{a d s}=-G_{2}^{a d s}$. This model can be used to simulate the equilibrium contact angles ranging from $30^{\circ}$ to $150^{\circ}$. Similarly for multiphase simulations we fix a wetting parameter, $\rho_{w}$ for the nodes in the wall and calculate the Shan-Chen force (5.27) at the wall [85]

$$
\mathbf{F}^{a d s}=-G \psi\left(\rho_{w}\right) \sum_{i} w_{i} s\left(\mathbf{x}+\mathbf{e}_{i}\right) \mathbf{e}_{i}
$$

where, $s\left(\mathbf{x}+\mathbf{e}_{i}\right)=1$ for a wall node and is 0 for a fluid node. The parameter $\rho_{w}$ is varied to control the equilibrium contact angle at the wall. This model can also simulate the equilibrium contact angles ranging from $30^{\circ}$ to $150^{\circ}$.

\subsection{Comparing the lubrication model and Lattice Boltzmann}

In this section we compare the results of the generalized lubrication (GL) model and the lattice Boltzmann (LB) simulations. Since the lattice Boltzmann simulation is limited to moderate viscosity ratios $R$, the comparison is done for $R=0.03,0.8$ and 1. We further explore the parameter space in Sec. 5.5, using only the lubrication approach. 
The results for this section are computed for $\theta_{e}=\pi / 2$ and $\alpha=\pi / 2$. The lattice separation in LB is 0.01 (in unit of capillary length), which will be related to an effective slip length $\lambda_{s}$ from the comparison with the lubrication model.

\subsubsection{Meniscus rise}

We first compare the meniscus rise $\Delta$ for $R=0.03,0.8$ and 1 in Fig. 5.3. When the plate is at rest, $\mathrm{Ca}=0$, the meniscus is perfectly horizontal $\Delta=0$ due to the choice of $\theta_{e}$ and $\alpha$. Let us first consider the case where both liquids have identical viscosity, $R=1$, but are still immiscible due to the nonzero surface tension. This case is perfectly symmetric in the sense that $\mathrm{Ca} \rightarrow-\mathrm{Ca}$ gives $\Delta \rightarrow-\Delta$ : there is no difference between plunging and withdrawing. This symmetry is indeed observed in Fig. 5.3. Blue circles represent LB simulations, while the dash-dotted line is the GL model. We use this symmetric case to calibrate the microscopic parameter of the GL model. A nearly perfect fit is achieved for slip length $\lambda_{s}=0.002$, which is a reasonable value given that the grid size used in the LB simulation is 0.01 . As $\mathrm{Ca}$ increases, the viscous forces increasingly deform the interface, leading to a change in $\Delta$.

It is interesting to see to what extent the same microscopic parameter $\lambda_{s}$ is able to describe different viscosity ratios. We first mildly decrease the viscosity ratio to $R=0.83$ and still find a very good agreement between LB an GL (red squares and dotted line respectively). With respect to the case $R=1$ we see that $\Delta$ is slightly smaller at a given value of $\mathrm{Ca}$. This means that for the same speed, the meniscus is deformed by a smaller amount due to the lower viscosity of the upper phase. When further decrease the viscosity ratio to $R=0.03$ (green diamonds, dashed line), some differences between LB and GL becomes apparent (the same value for $\lambda_{s}$ is maintained). The meniscus in GL is systematically below the value obtained in LB. Still, both models agree reasonably well and display very similar trends. In particular, we find that much larger values of $\mathrm{Ca}$ can be achieved due to the strong reduction of the viscosity in the upper phase. This effect is most pronounced for the plunging case, for which the liquid is advancing. This is consistent with experimental observations that advancing contact lines can move much more rapidly than receding contact lines [76]. The breakdown of the steady solutions, which signal the transition to air/liquid entrainment will be discussed in detail in Sec. 5.5.

\subsubsection{Shape of the meniscus}

A much more detailed test for the two models is to investigate the detailed structure of the interface: How well do the shapes of the menisci compare between GL and 


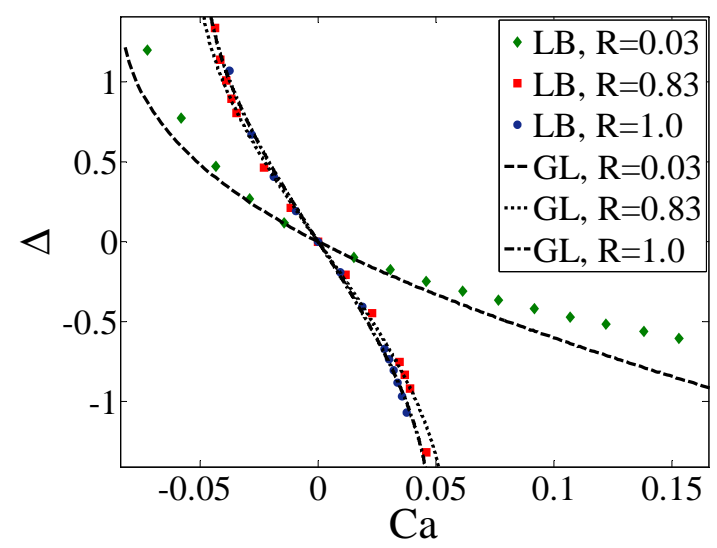

Figure 5.3: Meniscus deformation $\Delta$ as function of $\mathrm{Ca}$ for $\theta_{e}=\pi / 2$. Ca is negative/positive when the plate is moving upward/downward. Symbols: results of lattice Boltzmann (LB) simulation. Lattice separation $=0.01$. Curves: results of the lubrication-type (GL) model with slip length $\lambda_{s}=0.002$. All lengths are scaled by the capillary length $\ell_{\gamma}$.

LB? In Fig. 5.4, we show the dynamical meniscus profiles for $\mathrm{Ca}=0.019,0.028$ and 0.036 , in the case of equal viscosities, $R=1$. Note that the contact line position is held constant at $x=0$, such that the bath appears at different heights due to the increase in magnitude of $\Delta$ with speed. The agreement of the results of LT model and LB simulation is very good in particular for $\mathrm{Ca}=0.019$ and 0.028 , even down to the contact line region (Fig. 5.4b). For larger plate velocities some differences become apparent.

An even more detailed characterization of the meniscus shape is provided by the local angle of the interface $\theta$ vs $h$, see Fig. 5.5. Clearly, both the GL model and the LB simulation exhibit the same nontrivial variation of the contact angle. At small scales, the angle approaches $\theta_{e}=\pi / 2$, while at large scale the meniscus evolves towards the reservoir $\theta=\alpha=\pi / 2$. In between, the angle changes significantly due to the well-known effect of "viscous bending" [9]: the balance of viscosity and surface tension leads to a curvature of the interface. Very similar variations of the meniscus angle have been obtained experimentally [87]. 

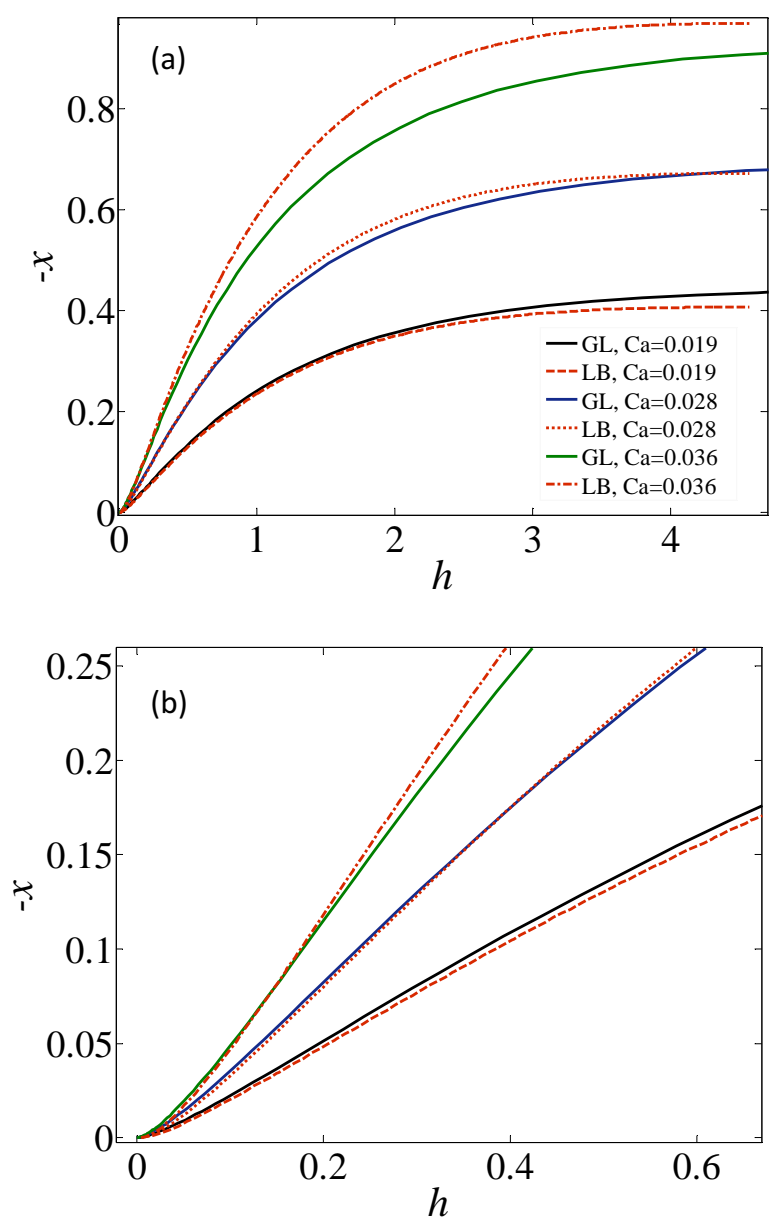

Figure 5.4: Dynamical meniscus profiles $-x$ vs $h$ for $R=1\left(\theta_{e}=\pi / 2\right.$ and $\lambda_{s}=$ 0.002 for the GL model, lattice separation $=0.01$ for the LB simulation). All lengths have been scaled by the capillary length. The contact line is at $x=0$ such that the bath is at different heights for different Ca. (a) Solid curves: results of GL model. Dashed/Dotted curves: results of LB simulation. (b) Zoom on the contact line region. 


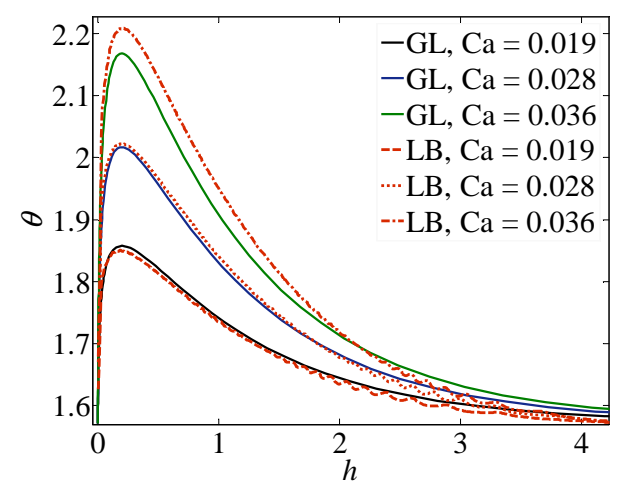

Figure 5.5: Local meniscus angle $\theta$ vs $h$ for $R=1$. Identical parameters as in Fig. 5.4.

\subsection{Maximum speeds and transition to air entrainment}

In this section we discuss the physics of air entrainment in the case of a plunging plate $(\mathrm{Ca}>0)$. For realistic situations the viscosity ratio $R$ is typically very small, of order $10^{-2}$ for water and much smaller for very viscous liquids. This regime can be accessed by the GL model only, as LB is restricted to moderate viscosity contrasts. In the first part of this section we discuss how the transition is captured in our model in terms of a bifurcation diagram. In the second part we investigate how the critical speed depends on the microscopic parameters such as the slip length and the static contact angle.

\subsubsection{Maximum speed for advancing contact lines}

We first consider a case where the equilibrium contact angle is close to $\pi$. For such a hydrophobic substrate we expect air entrainment to occur at relatively small $\mathrm{Ca}$ $[39,62]$, and therefore relatively weak curvature of the interface. This is important, since the assumption underlying the GL model is that the interface curvature is weak [29]. We will therefore focus on $\theta_{e}=2.8$ radians and explicitly verify how strongly the interface is curved for our numerical solutions.

Figure 5.6 shows the drop of the meniscus $\Delta$ as function of Ca for a viscosity ratio $R=0.01\left(\lambda_{s}=10^{-5}\right.$, i.e. of the order of $\left.10 \mathrm{~nm}\right)$. As Ca increases, the contact line equilibrates at a lower position resulting in a more negative value of $\Delta$. However, when $\mathrm{Ca}$ achieves a certain critical value, stationary solutions cease to exist. This corresponds to a maximum plate velocity, or critical capillary number $\mathrm{Ca}_{\mathrm{c}}$. By analogy to deposition of liquid films for plate withdrawal [12-16], this can be identified 


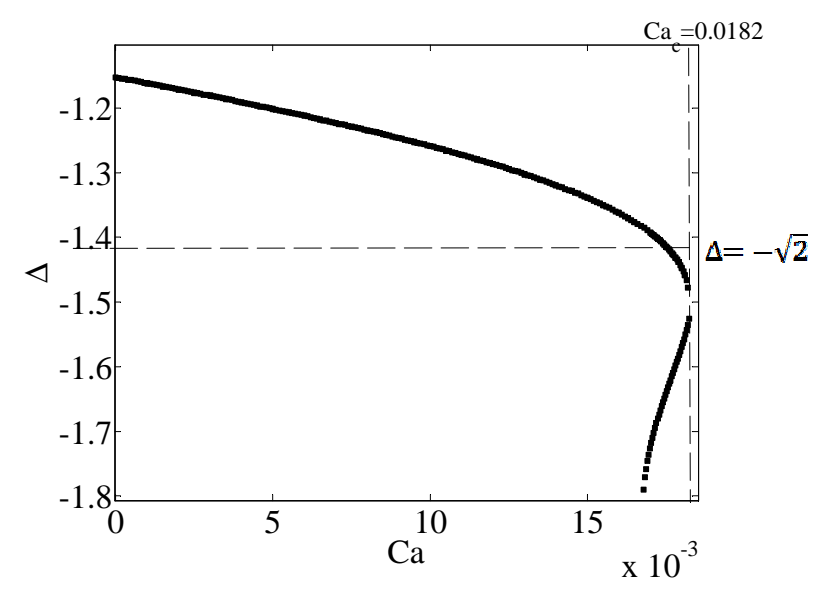

Figure 5.6: Meniscus fall $\Delta$ versus $\mathrm{Ca}\left(\theta_{e}=2.8\right.$ radians, $\left.R=0.01, \lambda_{s}=10^{-5}\right)$. The horizontal dashed line indicates the minimum value of $\Delta$ for a static meniscus (= $-\sqrt{2}$, with $\theta_{e}=\pi$ ).

as the onset of air entrainment: above $\mathrm{Ca}_{c}$, unsteady solutions will develop, with a downward motion of the contact line [76]. As can be seen in Fig. 5.6, the critical point arises close to $\Delta=-\sqrt{2}$, which according to (5.1) corresponds to a meniscus with apparent contact angle $\pi$. This is the analogue of the withdrawal case, for which $\Delta=+\sqrt{2}$ and the apparent contact angle vanishes at the transition [3,13]. Note that viscous effects push system slightly below this maximum extent of deformation for a perfectly static meniscus, with the critical point slightly below $\Delta=-\sqrt{2}$.

Interestingly, for a range of speeds $\mathrm{Ca}<\mathrm{Ca}_{\mathrm{c}}$ one actually finds more than one solution (cf. Fig. 5.6). Upon decreasing $\Delta$, the capillary number first increases and then decreases close to the critical point. We refer to the solution branches around $\mathrm{Ca}_{c}$ as the upper and lower branch respectively. Once again, an identical bifurcation structure was previously observed for the withdrawing plate case [13, 28]. To compare these two types of solutions, we show the corresponding meniscus profiles for $\mathrm{Ca}=0.017$ in Fig. 5.7. At a large distance from the contact line, the solutions are almost identical in shape. Zooming in on the contact line region, however, we see the lower branch (red dashed curve) solution displays a "finger" that explains the larger magnitude of $\Delta$ with respect to the upper branch. 


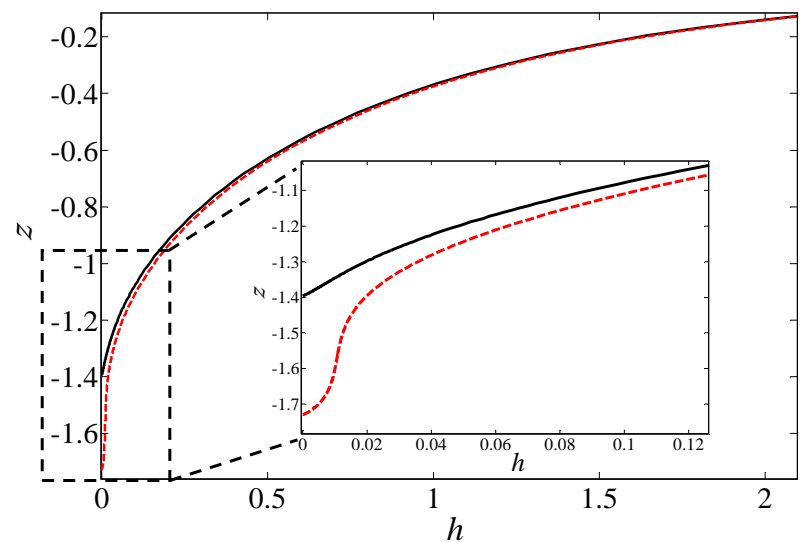

Figure 5.7: Meniscus profiles for the upper branch (black solid curve) and the lower branch (red dashed curve) solutions for $\mathrm{Ca}=0.017\left(\theta_{e}=2.8\right.$ radians, $R=0.01$, $\left.\lambda_{s}=10^{-5}\right)$. Here we define $z=\Delta-x$. So the bath level is at $z=0$.

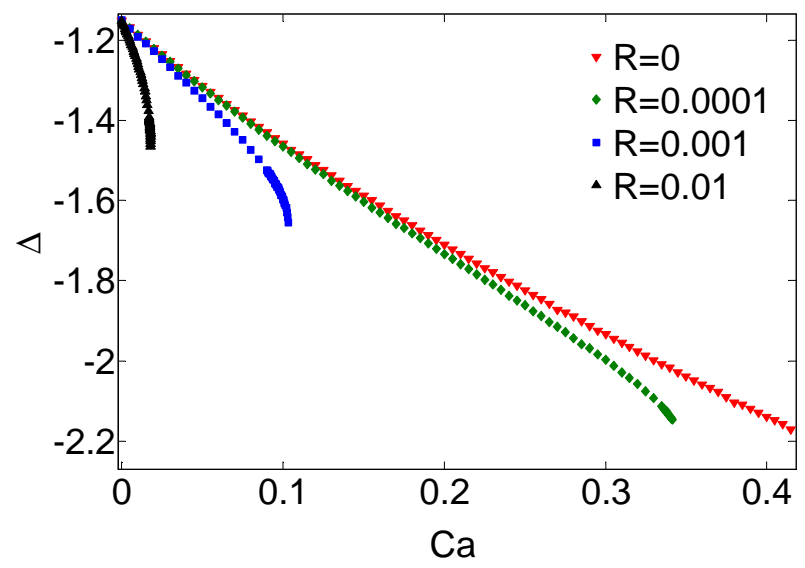

Figure 5.8: Meniscus fall $\Delta$ versus Ca for different viscosity ratios $\left(\theta_{e}=2.8\right.$ radians, $\lambda_{s}=10^{-5}$ ). For the case the gas phase has no viscosity, $R=0$, steady-state menisci can be maintained to arbitrarily large velocity (within our numerical resolution). 


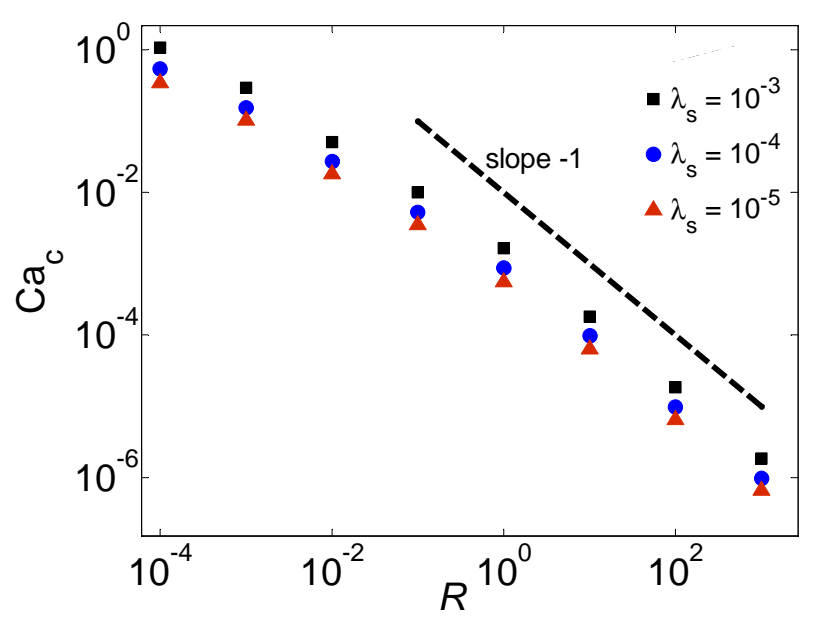

Figure 5.9: Critical capillary number $\mathrm{Ca}_{\mathrm{c}}$ versus viscosity ratio $R$ for different slip lengths $\lambda_{s}\left(\theta_{e}=2.8\right.$ radians $)$. The dashed line indicates a power law with exponent -1 , which is valid for large $R$.

\subsubsection{Effect of viscosity ratio}

A key parameter for the transition to air entrainment is the viscosity ratio $R$. Figure 5.8 shows the meniscus fall $\Delta$ as function of $\mathrm{Ca}$ for different viscosity ratios: $R=0,10^{-4}, 10^{-3}$ and $10^{-2}$. At $\mathrm{Ca}=0$, all cases have the same value of $\Delta=-1.15$ corresponding to a static bath with contact angle $\theta_{e}=2.8$. Now we consider the result for $R=0$, for which there are no viscous effects in the air $\left(\eta_{g}=0\right)$. We observe that $\Delta$ decreases with $\mathrm{Ca}$, but without any bifurcation. It appears that steady meniscus solutions can be sustained up to arbitrarily large plate velocities. In fact, the curve is consistent with the scaling $\Delta \sim-\sqrt{\mathrm{Ca}}$, corresponding to a simple balance between gravity and viscosity. For $R \neq 0$, however, the situation becomes fundamentally different. While the curves follow the same trend as for $R=0$ at small $\mathrm{Ca}$, a deviation appears at larger speed that ultimately leads to a critical point. Each nonzero viscosity ratio has a well-defined critical speed, with $\mathrm{Ca}_{c}$ increasing when the viscosity ratio $R$ is reduced.

These observations can be interpreted as follows. As long as the viscosity of the air has a negligible effect on the flow, the curves are indistinguishable from the case $R=0$. Deviations of the $R=0$ curve signal that the air flow starts to influence the shape of the meniscus. Physically, this arises because the interface slope approaches $\pi$, leaving only a narrow wedge angle for the air flow. Figure 5.2 illustrates that the 
recirculation in the air induces significant velocity gradients: despite the small air viscosity, the stresses in the small wedge of air become comparable to those in the liquid. Mathematically, this can be derived from the function $f(\theta, R)$ as defined in (5.16). For small $R$ and $\theta$ close to $\pi$, we can approximate:

$$
f(\theta, R) \simeq f(\theta, 0)-4 R \simeq-\frac{2(\pi-\theta)^{3}}{3 \pi}-4 R
$$

as long as $\pi-\theta \gg 2 \pi R$. The first term in (5.30) represents the (relative) viscous contribution inside the liquid, which vanishes in the limit $\theta \rightarrow \pi$. The second term represents the viscous contribution in the air, which will be significant once $(\pi-$ $\theta) \sim R^{1 / 3}$. Noting that the contact line is receding from the point of view of the air phase, one understands that a critical speed appears when the effect of the air becomes important.

The dependence of the critical speed $\mathrm{Ca}_{\mathrm{c}}$ on the viscosity ratio $R$ is shown in Fig. 5.9 (for various slip lengths). First we consider the limit $R \gg 1$, for which the upper fluid is actually much more viscous than the bottom fluid. This is the usual case of a receding contact line that is completely dominated by the upper (receding) phase. In this limit we expect the critical speed to scale with viscosity of the upper phase, denoted $\eta_{g}$, such that $U_{c} \sim \gamma / \eta_{g}$. Since we have based the capillary number on the viscosity $\eta_{\ell}$, we obtain $\mathrm{Ca}_{c} \equiv U_{c} \eta_{\ell} / \gamma \sim R^{-1}$. This is indeed observed in Fig. 5.9 at $R \gg 1$. However, our main interest here lies in the opposite limit, i.e. $R \ll 1$, as for air entrainment. As already mentioned, the critical speed seems to increase indefinitely by reducing the viscosity ratio. This suggests that for the limiting case of $R=0$, steady menisci can be sustained at arbitrarily large plunging velocities. Our numerical resolution does not allow for a perfect determination of the asymptotics for $R \ll 1$. Enforcing a power law fit, $\mathrm{Ca}_{c} \sim R^{\beta}$, in the range $R=10^{-4}-10^{-1}$, one obtains $\beta=-0.67$. This (effective) exponent suggests that both phases play an important role in determining the critical speed. Namely, the exponent would be $\beta=-1$ if only $\eta_{g}$ were important, while $\beta=0$ corresponds to the case where $\eta_{\ell}$ is the only relevant viscosity.

Finally, we briefly verify the assumption of small curvature, necessary for the strict validity of the model. In Fig. 5.10 we plot the dimensionless curvature, $h|d \theta / d s|$, as function of $h$ in the vicinity of the critical point $\left(R=10^{-5}, 10^{-4}\right.$ and $10^{-3}, \lambda_{s}=$ $10^{-5}$ ). At small scales, $h|d \theta / d s| \ll 1$ for all $\mathrm{Ca}$, consistent with the assumption of small curvature. However, the curvature increases significantly when approaching the bath due to the bending of interface from a large contact angle to $\pi / 2$. The magnitude is acceptable in this regime, in particular since viscous effects becomes less important at large scales. Inclining the plate angle to values close to $\pi$ would further reduce this bending effect, and extend the range of validity of the GL model. 


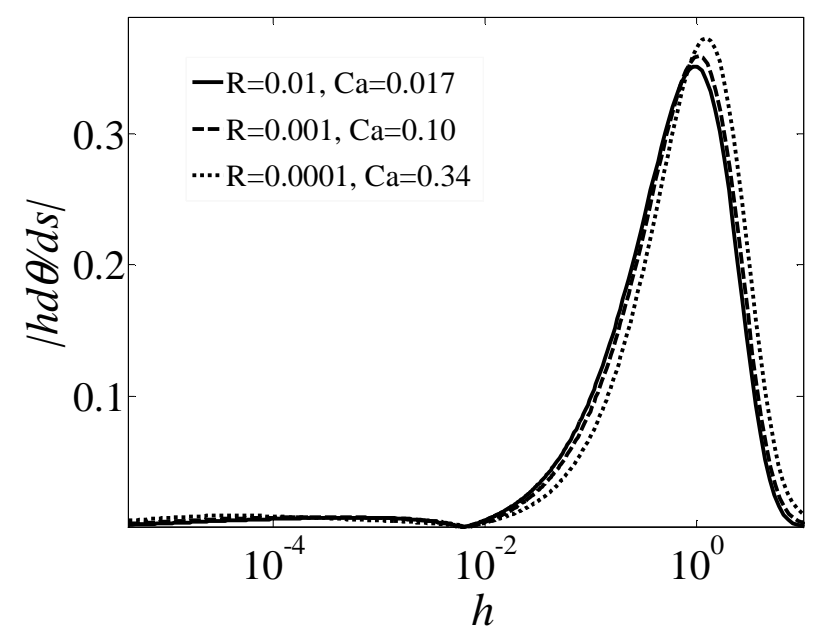

Figure 5.10: Scaled curvature $|h d \theta / d s|$ vs $h$ for Ca very close to $\mathrm{Ca}_{\mathrm{c}}\left(\lambda_{s}=10^{-5}\right)$.

\subsubsection{Dependence of the critical speed on microscopic parameters}

Apart from the viscosity ratio, the GL model contains two parameters: the slip length $\lambda_{s}$ and the microscopic (equilibrium) contact angle $\theta_{e}$. Here we discuss the dependence of $\mathrm{Ca}_{\mathrm{c}}$ on these parameters. The slip length was varied already in Fig. 5.9, with values $\lambda_{s}=10^{-5}, 10^{-4}$ and $10^{-3}$. As expected for wetting problems, we see a weak increase of $\mathrm{Ca}_{\mathrm{c}}$ with $\lambda_{s}$. A larger $\lambda_{s}$ reduces the range over which viscous dissipation is effective. This leads to a (logarithmic) reduction of the viscous dissipation, while the capillary driving remains unaltered. Hence, larger velocities can be achieved before air entrainment occurs.

The dependence of the critical speed on $\theta_{e}$ is investigated in Fig. 5.11. The figure reveals that there is no obvious universal scaling behavior for $\mathrm{Ca}_{c}$ down to viscosity ratios as small as $R=10^{-4}$. We have not been able to extend our numerical result to lower $R$. Enforcing a power-law fit, different $\theta_{e}$ would give rise to different exponents. We do clearly see that $\mathrm{Ca}_{\mathrm{c}}$ decreases with $\theta_{e}$, which is further emphasized in Fig. 5.12. Consistent with [39,62], the critical speed vanishes in the hydrophobic limit where $\theta_{e} \rightarrow \pi$. We note that for contact angles that are not close to $\pi$, the shape of the meniscus displays significant curvatures. In this sense, we expect that our results are not fully quantitative solutions of the Stokes flow problem. 


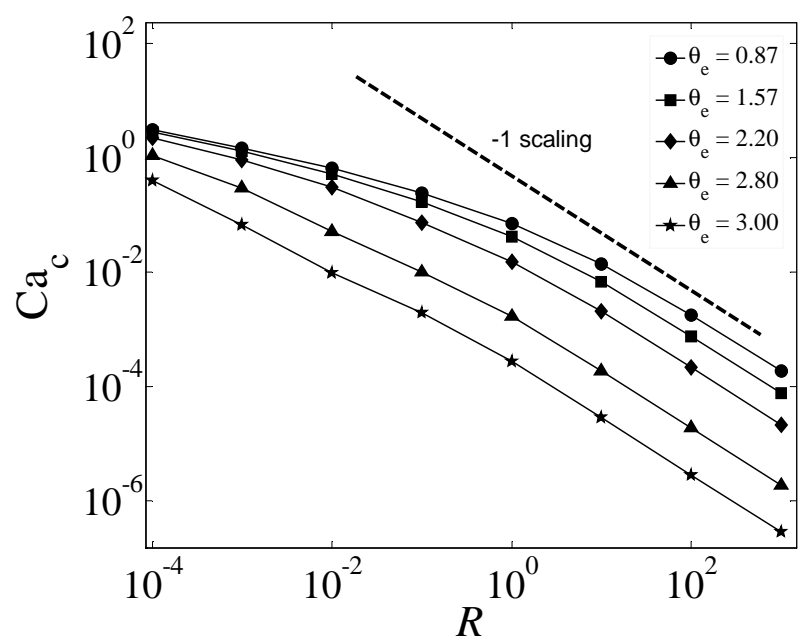

Figure 5.11: Critical speed $\mathrm{Ca}_{\mathrm{c}}$ as a function of $R$ for different static contact angle $\theta_{e}$ $\left(\lambda_{s}=0.001\right)$.

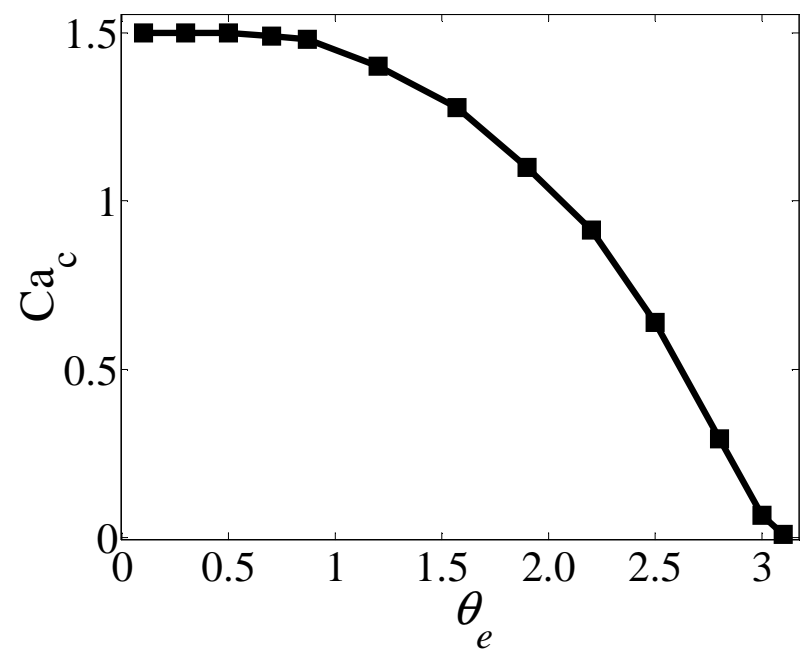

Figure 5.12: Critical speed $\mathrm{Ca}_{\mathrm{c}}$ as a function of static contact angle $\theta_{e}$ for $R=0.001$ and $\lambda_{s}=0.001$. 


\subsection{Discussion}

In this Chapter, the meniscus deformation in a dip-coating geometry has been studied using two models, namely the generalized lubrication model (GL) and the lattice Boltzmann simulation (LB). The predictions of these two models are in good agreement, in particular when $\mathrm{Ca}$ is relatively small. When exploring larger values of $\mathrm{Ca}$, the two models start to differ as can be seen in Fig. 5.3, which can be attributed to different physics at microscopic scales. Yet, qualitative features, such as the bending of the meniscus and the dependence on viscosity ratio, are comparable in both models. It turned out both models have difficulties in accessing even larger values of $\mathrm{Ca}(\mathrm{Ca} \gtrsim 1)$. For the GL model, the validity is limited by its assumption of small interface curvature, which is naturally violated at large Ca. Still, the transition to air entrainment for $\theta_{e}$ close to $\pi$ involves relatively weak curvatures and is thus captured by the GL model. For the LB simulations the main challenge is that the problem contains multiple length scales and hence requires a large number of grid points. In particular, the present simulations are not able to capture the transition to air entrainment.

In the second part of this Chapter, the critical speed of air entrainment was investigated by the GL model. We have found a strong dependence of critical speeds on the air viscosity, which is consistent with the experiments discussed in the previous Chapter. Remarkably, both our theoretical and experimental results differ from Cox's model in which $\mathrm{Ca}_{\mathrm{c}}$ is predicted to depend only logarithmically on air viscosity [21]. Interestingly, such a weak logarithmic relation was also observed in the case of liquid impacting on liquid $[19,20]$, for which there is no moving contact line. Our results therefore suggest that the mechanism leading to air entrainment can be fundamentally different depending on whether a contact line is present or not.

The direct comparison of the GL model with our experiments was already given in Fig. 4.3 of Chapter 4. The solid line is the prediction from our model using realistic estimates of the microscopic parameters (with different values for the liquid and air, see Supplementary Material in Chapter 4), and in this sense is free from fitting parameters. Clearly, the model captures qualitatively how $\mathrm{Ca}_{\mathrm{c}}$ depends on the viscosity ratio $\eta_{g} / \eta_{\ell}$. In particular, the critical speed does not depend solely on the liquid viscosity (in which case $\mathrm{Ca}_{c}$ would be constant), nor does it depend only on the gas viscosity (in which case $\mathrm{Ca}_{c} \sim \eta_{\ell} / \eta_{g}$ ). Quantitatively, however, the agreement is not satisfactory. We believe this is due to the relatively large meniscus curvatures encountered in the experiments (static contact angle of the substrate $\approx 50^{\circ}$ ), pushing the problem beyond the assumptions of the model. It would be interesting to explore other methods to achieve a more quantitative description of air entrainment by advancing contact line, in particular for large values of $\mathrm{Ca}$. From an experimental 
perspective, more insight could be obtained by varying the gas viscosity or by replacing the air by a liquid of low viscosity. It would also be interesting to perform experiments with a substrate of large static contact angles. 


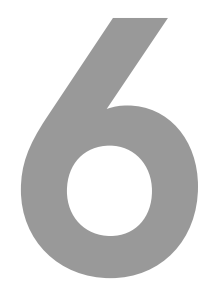

\section{Withdrawing a plate from a liquid with small viscosity: effect of inertia}

\subsection{Introduction}

In the previous Chapters, we focused on flows in a range of small Reynolds number $R e$, thus the effect of inertia was not taken into consideration. This is a natural limit for wetting dynamics, since close to the contact line the flow is always viscosity dominated. However, some very common fluids (e.g. water) have a relatively low viscosity and inertia can be important at scales away from the contact line. As an example we mention Immersion Lithography [4], where the fluid in between the lens and the substrate is water that has viscosity $\eta$ around $10^{-3} \mathrm{mPa} \cdot \mathrm{s}$ and density $\rho=$ $10^{3} \mathrm{~kg} / \mathrm{m}^{3}$ at room temperature. If we take the typical speed of flow $U$ as $0.1-1$ $\mathrm{m} / \mathrm{s}$ and the distance between the lens and the substrate $L$ as $0.1-1 \mathrm{~mm}$, then the Reynolds number based on the macroscopic dimension is of order $\operatorname{Re} \equiv \rho U L / \eta \simeq 10$, or larger. Indeed, wetting experiments on water exhibit some quantitative disparities with respect to predictions of viscous theory [1, 4]: (i) the critical speed is lower than expected, by about a factor 2 , (ii) the dynamic contact angle varies more quickly with speed than predicted by the Cox-Voinov relation [21, 27], again by approximately a

\footnotetext{
${ }^{*}$ Manuscript to be submitted: T.S. Chan and J.H. Snoeijer, Withdrawing a plate from a liquid with small viscosity: effect of inertia.
} 
factor 2. This contrasts the level of agreement with viscous liquids such as silicon oils, for which quantitative agreement is achieved up to $20 \%$ [1, 13, 14]. From a technological perspective, such quantitative difference is significant. It has remained unclear whether this effect can indeed be attributed to the relatively large Reynolds numbers encountered for water.

In this Chapter we provide a simple model to include the effect of inertia. We follow the idea of Koulago et al. [88], used in the context of Landau-Levich films, to consider a depth-integrated momentum balance that incorporates inertial effects. Under the standard assumptions where the thickness of the liquid film is slowly varying, the momentum equations can be reduced to the classical boundary layer equations $[89,90]$, with transport mainly in the direction along the wall. The standard lubrication approximation is recovered by integrating over the layer thickness using a parabolic velocity profile. Here we perform the same procedure, but keeping the integrals over the inertial terms. The result is a modified lubrication equation, that includes a new term proportional to the Reynolds number. Below we first derive this equation, and discuss under what conditions inertia is expected to play a role in wetting flows. Then we make a quantitative analysis, by solving the equation in a dip-coating geometry for receding contact lines (plate withdrawal). We show that inertial effects tend to lower the critical speed of dynamical wetting transition, and we quantify this dependence in terms of contact angle and dimensionless parameters.

\subsection{Lubrication equation including inertia effect}

We consider a smooth, chemically homogeneous solid plate being withdrawn from a liquid reservoir at a constant speed $U_{p}$. Since the dynamics of the air is negligible, we focus on single-phase flow only. The plate is inclined at a small angle $\alpha$ with the horizontal, as sketched in Fig. 6.1. For $U_{p}=0$, there is no flow in the fluid, and the shape of the interface is determined by the balance between capillarity and gravity. When the plate is moving, $U_{p}>0$, flow is generated in the fluid. The contact line rises and then achieves a new equilibrium position above the static one as long as $U_{p}$ is below a critical value $U_{c}$. We assume that the contact line is straight and perpendicular to the movement of the plate, so the problem can be treated as two-dimensional. For steady states $\left(U_{p}<U_{c}\right)$, we characterize the deformation of the interface by a meniscus rise $z_{c l}$, the distance of the contact line above the bath, see Fig. 6.1. The interface shape is described by $h(x)$, and the velocity field $\vec{u}=u(x, y) \hat{x}+v(x, y) \hat{y}$ can be determined by solving the Navier-Stokes equations with appropriate boundary conditions. The Navier-Stokes equations for incompressible fluid of viscosity $\eta$, 


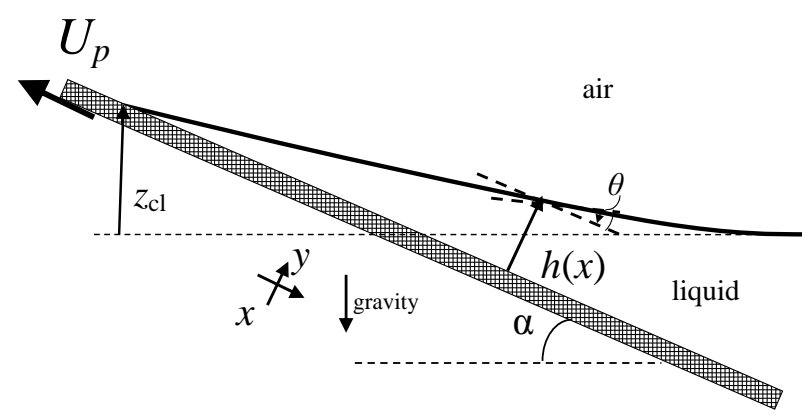

Figure 6.1: Schematic diagram of a plate being withdrawn from a liquid reservoir at a constant speed $U_{p}$. The plate is inclined at a small angle $\alpha$ with the horizontal. The shape of the interface is described by $h(x)$, where $x$ is measured relative to the contact line position. The deformation of the interface is characterized by a meniscus rise $z_{c l}$.

density $\rho$ read

$$
\begin{aligned}
& \rho\left(u \cdot \frac{\partial u}{\partial x}+v \cdot \frac{\partial u}{\partial y}\right)=-\frac{\partial p}{\partial x}+\eta\left(\frac{\partial^{2} u}{\partial x^{2}}+\frac{\partial^{2} u}{\partial y^{2}}\right)+\rho g \sin \alpha, \\
& \rho\left(u \cdot \frac{\partial v}{\partial x}+v \cdot \frac{\partial v}{\partial y}\right)=-\frac{\partial p}{\partial y}+\eta\left(\frac{\partial^{2} v}{\partial x^{2}}+\frac{\partial^{2} v}{\partial y^{2}}\right)-\rho g \cos \alpha
\end{aligned}
$$

where $p$ is the pressure and $g$ the gravitational acceleration. The continuity equation is given by

$$
\frac{\partial u}{\partial x}+\frac{\partial v}{\partial y}=0 .
$$

The boundary conditions at the wall $(\mathrm{y}=0)$ are

$$
v=0
$$

and

$$
u=\lambda_{s} \frac{\partial u}{\partial y}-U_{p} .
$$

Since imposing a no-slip boundary condition leads to infinite dissipation when approaching the contact line [6], a Navier slip boundary condition is applied. Note that $\lambda_{s}$ is the slip length, which is usually of the order of a nanometer.

At the steady interface, $y=h(x)$, we can impose

$$
\frac{v}{u}=h^{\prime},
$$


which means the flow is parallel to the interface. Shear stress vanishes at the interface,

$$
\hat{t} \cdot \sigma \cdot \hat{n}=0
$$

and the normal stress is related to the interface curvature $\kappa$ and the surface tension $\gamma$ by the Laplace's law

$$
\hat{n} \cdot \sigma \cdot \hat{n}=\gamma \kappa .
$$

Here the stress tensor $\sigma$ and the unit vectors $\hat{n}$ and $\hat{t}$ are defined by

$$
\begin{gathered}
\sigma=\left(\begin{array}{cc}
-p+2 \eta \frac{\partial u}{\partial x} & \eta\left(\frac{\partial u}{\partial y}+\frac{\partial v}{\partial x}\right) \\
\eta\left(\frac{\partial u}{\partial y}+\frac{\partial v}{\partial x}\right) & -p+2 \eta \frac{\partial v}{\partial y}
\end{array}\right), \\
\hat{t}=\cos \theta \hat{x}+\sin \theta \hat{y}
\end{gathered}
$$

and

$$
\hat{n}=-\sin \theta \hat{x}+\cos \theta \hat{y},
$$

where $\theta$ is the local angle of the interface, see Fig. 6.1.

In the following we will simplify the above governing equations using the lubrication approximation, namely the slope of the interface is small, i.e. $h^{\prime}(x) \ll 1$. For details of the derivation, we refer to [10, 88]. In this framework, the Navier-Stokes equations reduce to

$$
\begin{aligned}
\rho\left(u \cdot \frac{\partial u}{\partial x}+v \cdot \frac{\partial u}{\partial y}\right) & =-\frac{\partial p}{\partial x}+\eta \frac{\partial^{2} u}{\partial y^{2}}+\rho g \sin \alpha, \\
\frac{\partial p}{\partial y} & =-\rho g \cos \alpha .
\end{aligned}
$$

The boundary conditions of the stresses at the steady interface, $y=h(x)$, can be simplified as

$$
\frac{\partial u}{\partial y}=0
$$

and

$$
\gamma h^{\prime \prime}=-p .
$$

Solving (6.12) and (6.13) for the flow filed is very challenging due to the inertial terms $u \frac{\partial u}{\partial x}+v \frac{\partial u}{\partial y}$ in (6.12). Without the inertial term, the flow can be easily found to be parabolic. Here we adopt the approach used in [88], where the flow is assumed to 
remain parabolic, to consider a depth-integrated momentum balance that incorporates inertial effects. We write the velocity field as

$$
u=U_{p}\left[\frac{3\left(1+\frac{q}{h}\right)}{2 h\left(h+3 \lambda_{s}\right)}\left\{2 h\left(y+\lambda_{s}\right)-y^{2}\right\}-1\right],
$$

where $q$ is the flux defined by

$$
q \equiv \int_{0}^{h} u d y
$$

Note that this flow fulfills the boundary conditions (6.5) and (6.14).

Now we are in a position to obtain an equation for the interface profile $h(x)$. First we integrate both sides of (6.12) with respect to $y$ from $y=0$ to $y=h$, this leads to

$$
\frac{d \int_{0}^{h} u^{2} d y}{d x}=\left(-\frac{\partial p}{\partial x}+\rho g \sin \alpha\right) h-\left.\frac{\partial u}{\partial y}\right|_{y=0} .
$$

Note that $\partial p / \partial x$ is independent of $y$ due to (6.13). The left hand side of (6.18) was obtained as follows:

$$
\begin{array}{r}
\int_{0}^{h}\left(u \cdot \frac{\partial u}{\partial x}+v \cdot \frac{\partial u}{\partial y}\right) d y \\
=\int_{0}^{h} u \cdot \frac{\partial u}{\partial x} d y+\left.v \cdot u\right|_{0} ^{h}-\int_{0}^{h} u \cdot \frac{\partial v}{\partial y} d y \\
=\int_{0}^{h} u \cdot \frac{\partial u}{\partial x} d y+\left.u^{2} h^{\prime}\right|_{y=h}+\int_{0}^{h} u \cdot \frac{\partial u}{\partial x} d y \\
=\int_{0}^{h} \frac{\partial u^{2}}{\partial x} d y+\left.u^{2} h^{\prime}\right|_{y=h} \\
=\frac{d \int_{0}^{h} u^{2} d y}{d x} .
\end{array}
$$

Above we have used the continuity equation (6.3) and the boundary condition (6.4) and (6.6).

Next we substitute the expression (6.16) for the velocity field $u$ to (6.18). Since we investigate the stationary shape of the meniscus with the contact line remaining still with respect to the reservoir, we set $q=0$. Then (6.18) leads to

$$
\frac{\rho U_{p}^{2} h^{\prime} k\left(\lambda_{s} / h\right)}{5}=\left(-\frac{\partial p}{\partial x}+\rho g \sin \alpha\right) h-\frac{3 \eta U_{p}}{h+3 \lambda_{s}},
$$

where the function $k(s)$ is defined as

$$
k(s) \equiv \frac{1+9 s}{(1+3 s)^{3}} .
$$


We rewrite (6.24) in the following form

$$
-\frac{\partial p}{\partial x}=\frac{3 \eta U_{p}}{h\left(h+3 \lambda_{s}\right)}+\frac{\rho U_{p}^{2} h^{\prime} k\left(\lambda_{s} / h\right)}{5 h}-\rho g \sin \alpha .
$$

Next we make use of (6.13) and (6.8) to obtain an expression for $\partial p / \partial x$ in terms of the interface curvature. First we integrate (6.13) once with respect to $y$, this gives

$$
p=\rho g \cos \alpha y+f(x),
$$

where $f(x)$ is an undetermined function independent of $y$. Evaluating the pressure of expression (6.27) at the interface, i.e. $y=h$, and compare it to the boundary condition (6.15), then $f(x)$ is found to be

$$
f(x)=-\gamma h^{\prime \prime}-\rho g h \cos \alpha .
$$

Substituting (6.28) back to (6.27), the pressure can be written as

$$
p=-\gamma h^{\prime \prime}+\rho g(y-h) \cos \alpha .
$$

Differentiating (6.29) with respect to $x$, we obtain

$$
\frac{\partial p}{\partial x}=-\gamma h^{\prime \prime \prime}-\rho g h^{\prime} \cos \alpha
$$

Substituting (6.30) to (6.26), we end up with an interface equation

$$
\gamma h^{\prime \prime \prime}=\frac{3 \eta U_{p}}{h\left(h+3 \lambda_{s}\right)}+\frac{\rho U_{p}^{2} h^{\prime} k\left(\lambda_{s} / h\right)}{5 h}-\rho g\left(h^{\prime} \cos \alpha+\sin \alpha\right) .
$$

At macroscopic scales the capillary force dominates the viscous force, hence the natural length scale is the capillary length $\ell_{c} \equiv \sqrt{\gamma / \rho g}$. In the following we write all the lengths in terms of the capillary length. For simplicity of notations, we use the same symbols for the rescaled lengths. Thus the interface equation (6.31), after rescaling the lengths, becomes

$$
h^{\prime \prime \prime}=\frac{3 \mathrm{Ca}}{h\left(h+3 \lambda_{s}\right)}\left(1+\frac{\operatorname{Rehh}^{\prime} \tilde{k}\left(\lambda_{s} / h\right)}{15}\right)+h^{\prime}-\alpha,
$$

where the Reynolds number $R e$ is defined by

$$
R e=\rho U_{p} \ell_{c} / \eta
$$


the capillary number

$$
\mathrm{Ca}=\frac{\eta U_{p}}{\gamma}
$$

and $\tilde{k}(s)=k(s)(1+3 s)$. Note that we also took the limit of small $\alpha$. (6.32) indeed is the same as the interface equation in [88] except here we consider the case $q=0$, and include the slip length and gravity.

Here we discuss some properties of (6.32). We see the inertia term is relevant, with respect to the viscous term, only when $R e h h^{\prime}$ is order of unity or larger. In the viscous regime this term can be neglected, one recovers the standard lubrication equation. Moreover, we see that the correction term due to inertia contains a factor $h^{\prime}$, which in the lubrication framework, is of small order. Thus we expect the effect of inertia will be significant only when $R e$ is much larger than unity. In addition, the factor $h$ implies that in the region very close to the contact line, the viscous effect dominates the inertia effect.

Since in experiments it is more common to vary the plate velocity with physical parameters fixed, it is more convenient to write the interface equation in terms of $\mathrm{Ca}$ and a speed-independent parameter known as Ohnesorge number $\mathrm{Oh} \equiv \sqrt{\mathrm{Ca} / \mathrm{Re}}=$ $\eta / \sqrt{\rho \ell_{c} \gamma}$. Note that Oh goes as $1 / \sqrt{R e}$. We then rewrite (6.32) in the following form

$$
h^{\prime \prime \prime}=\frac{3 \mathrm{Ca}}{h\left(h+3 \lambda_{s}\right)}\left(1+\frac{\mathrm{Ca} h h^{\prime} \bar{k}\left(\lambda_{s} / h\right)}{15 \mathrm{Oh}^{2}}\right)+h^{\prime}-\alpha .
$$

\subsection{Results}

We now investigate the influence of the inertial term on the critical speed for the dynamical wetting transition in dip-coating. We first consider a case of very small contact angle, $\theta_{e}=0.05$ radian, small plate inclination $\alpha=0.1$ radian, and $\lambda_{s}=10^{-4}$. As in the previous Chapters, the boundary condition at the contact line is $h^{\prime}=\theta_{e}$, while the interface joins the reservoir at large distance, $h^{\prime} \rightarrow \alpha$.

Figure 6.2 compares meniscus profiles with and without inertial effects, by plotting $h^{\prime}$ as function of $x$ for $\mathrm{Ca}=3 \times 10^{-6}$. The solid curve corresponds to $1 / \mathrm{Oh}=0$, which has no inertia. At small distance to the contact line, we see that the interface first decreases below the equilibrium value, due to the strong viscous forces. At larger scales, $h^{\prime}$ increases to match the boundary condition at the bath. Since both the velocities and the interface slopes are very small, it turns out that inertial effects only appear for very large values of $1 / \mathrm{Oh}$. The dashed curve in Fig. 6.2 is for $1 / \mathrm{Oh}=10^{5}$. As expected, there is no difference with the profile obtained for $1 / \mathrm{Oh}=0$ at small 
scales. However, at larger scales one observes that the inertial effect induces an additional "bending" of the interface, resulting in a further decrease in slope.

Effectively we may think of the additional bending as a lowering of the apparent contact angle, or as an increased resistance to flow. Hence, we expect the critical speed of the dewetting transition to decrease due to inertia. To determine the critical speed, we characterize the deformation of the interface by the meniscus rise $z_{c l}$, defined in Fig. 6.1. The meniscus rise is plotted as a function of Ca in Fig. 6.3, for $1 / \mathrm{Oh}=0,3 \times 10^{4}$ and $10^{5}$. For the non-inertial case ( $1 / \mathrm{Oh}=0$, circles), $z_{c l}$ increases with $\mathrm{Ca}$ until at a certain maximum value. Above this critical $\mathrm{Ca}_{c}$, there are no steady-state solutions and a film will be deposited. For $1 / \mathrm{Oh}=3 \times 10^{4}$ (triangles), $z_{c l}$ coincides with that for $1 / \mathrm{Oh}=0$ when $\mathrm{Ca}$ is relatively small. At higher $\mathrm{Ca}, z_{c l}$ rises above the curve for $1 / \mathrm{Oh}=0$, that means inertia tends to elongate the interface. As anticipated, the critical speed is lowered with respect to the case $1 / \mathrm{Oh}=0$, although the effect is relatively small. However, the trend is enhanced when $1 / \mathrm{Oh}$ is further increased up to $10^{5}$ (squares). This corresponds to a Reynolds number $R e=3 \times 10^{4}$ with $U_{p}$ based on the critical speed.

The dependence of the critical speed can be summarized by plotting $\mathrm{Ca}_{\mathrm{c}}$ as function of $1 / \mathrm{Oh}$ (Fig. 6.4). We see inertia starts to have effect only when $1 / \mathrm{Oh}$ is as large as $10^{4}$. As mentioned above, this is due to the small contact angles. To have an impression how such a large magnitude appears, we make an estimation as follows. From (6.35), the inertial term is important when $\mathrm{Ca}^{\prime} h^{\prime} / 15 \mathrm{Oh}^{2} \gtrsim 1$. If we take $\mathrm{Ca}=\mathrm{Ca}_{\mathrm{c}} \approx \theta_{e}^{3} / 9 \ln (L / \ell)[21,27]$ (where $L / \ell$ is a ratio between the macroscopic length and the microscopic length, here we take the ratio between the capillary length and the slip length, for our numerical calculations, which equals to $10^{4}$ ); $h^{\prime}=\theta_{e}=0.05$, and $h$ is estimated by balancing gravity and the viscous term, i.e. $h \sim \sqrt{3 \mathrm{Ca}} / \alpha$. Hence we will obtain $1 / \mathrm{Oh} \gtrsim 2 \times 10^{5}$, which is consistent with our numerical result. It is interesting to see how the critical speed changes as the contact angle is enhanced. We therefore repeated our calculations for larger values of the contact angle. For large contact angles, $h^{\prime \prime}$ in (6.35) is not a correct expression for the curvature anymore, thus we replace it by a full expression of curvature, which is

$$
\kappa=\frac{h^{\prime \prime}}{\left(1+h^{\prime 2}\right)^{3 / 2}} .
$$

The result of the critical speed as a function of $\theta_{e}$ is shown in Fig. 6.5, in which circles correspond to $1 / \mathrm{Oh}=0$ and triangles are for $1 / \mathrm{Oh}=3 \times 10^{2}$. We see the critical speed increases with $\theta_{e}$. At small contact tangles ( $<0.5$ radian), $\mathrm{Ca}_{\mathrm{c}}$ is the same for $1 / \mathrm{Oh}=0$ and $1 / \mathrm{Oh}=3 \times 10^{2}$. Difference in critical speeds become more apparent as $\theta_{e}$ is further enhanced. When $\theta_{e}$ is up to 1.2 radian, the critical speed for $1 / \mathrm{Oh}=3 \times 10^{2}$ is around half of that for $1 / \mathrm{Oh}=0$. 


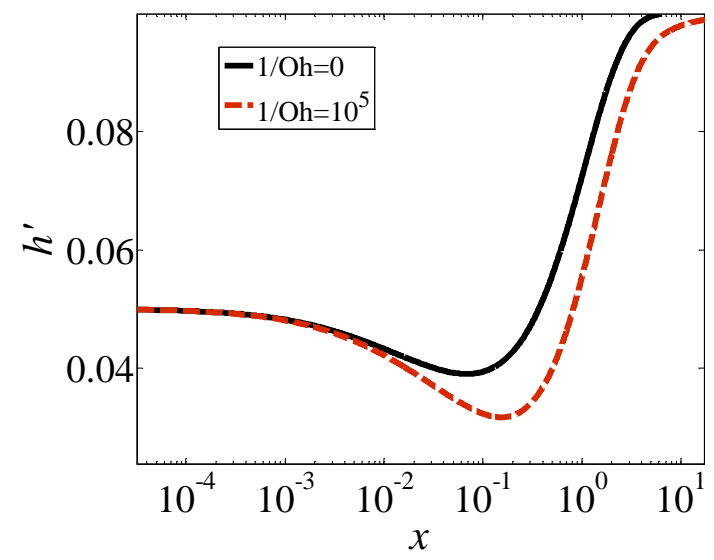

Figure 6.2: $h^{\prime}$ vs $x$ for $\mathrm{Ca}=3 \times 10^{-6}$. Solid line: $1 / \mathrm{Oh}=0$, Dashed line: $1 / \mathrm{Oh}=10^{5}$ $\left(\theta_{e}=0.05\right.$ radian, $\alpha=0.1$ radian and $\left.\lambda_{s}=10^{-4}\right)$.

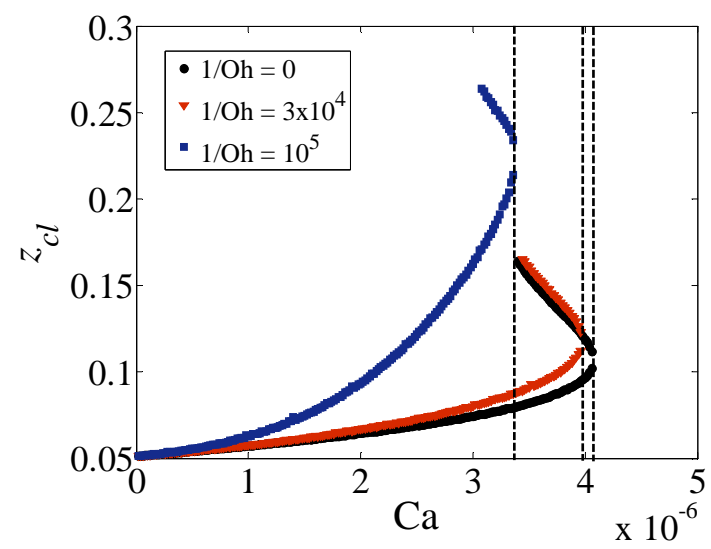

Figure 6.3: $z_{c l}$ vs $\mathrm{Ca}$ for $1 / \mathrm{Oh}=0,3 \times 10^{4}$ and $10^{5}\left(\theta_{e}=0.05\right.$ radian, $\alpha=0.1$ radian and $\lambda_{s}=10^{-4}$ ). Dashed vertical lines indicate the position of $\mathrm{Ca}_{\mathrm{c}}$.

\subsection{Discussion}

In this Chapter we provided a simple approach to investigate the effect of inertia on contact line motion. Following [88], we assumed that the velocity profile in the liquid remains parabolic and considered a depth-integrated momentum balance. The motivation for this work is that the breakup of water drops at the receding contact line 


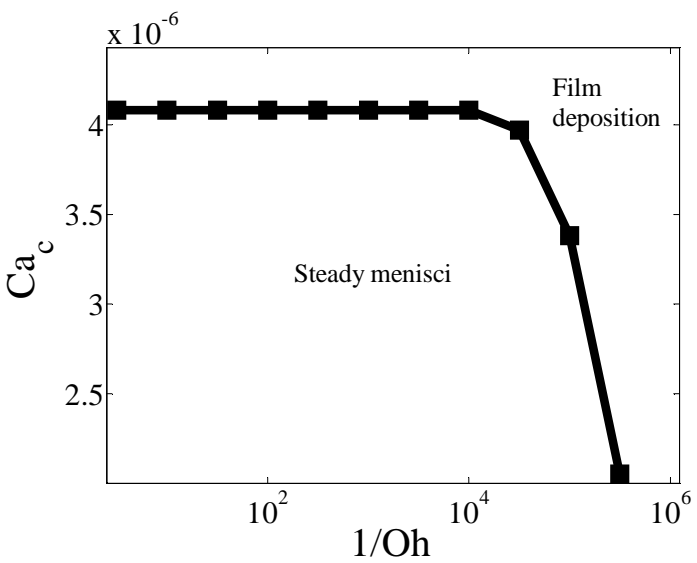

Figure 6.4: $\mathrm{Ca}_{\mathrm{c}}$ vs $1 / \mathrm{Oh}\left(\theta_{e}=0.05\right.$ radian, $\alpha=0.1$ radian and $\left.\lambda_{s}=10^{-4}\right)$.

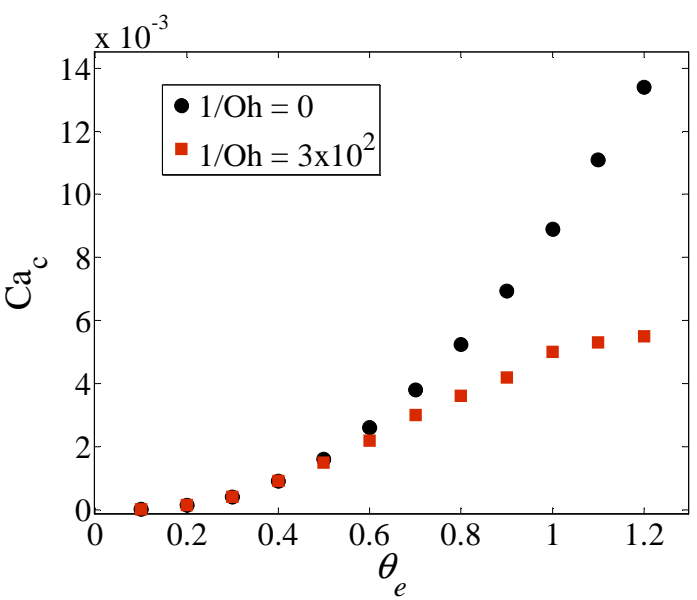

Figure 6.5: $\mathrm{Ca}_{\mathrm{c}}$ as a function of $\theta_{e}$ for $1 / \mathrm{Oh}=0$ and $1 / \mathrm{Oh}=3 \times 10^{2}(\alpha=0.1$ radian and $\lambda_{s}=10^{-4}$ ).

appears at much lower velocities than expected from viscous hydrodynamical theory: the speed was found to be smaller by approximately a factor $2[1,4]$, which cannot be explained using realistic values for the microscopic (slip) length. The results of this Chapter clearly show that inertial effects can indeed lower the critical speed. Interestingly, inertia is only effective at relatively large Reynolds numbers, $R e \gg 1$. 
This is because the relevant factor for inertial effects with respect to viscous forces is $\sim R e h h^{\prime}$, where $h$ is the local thickness (expressed in capillary length) and $h^{\prime}$ the interface slope. The influence of inertia is thus greatly suppressed for small contact angle. In addition, since viscous effects are always dominant at small distances from the contact line, where most of the dissipation occurs, the influence of inertial only appears at larger scales.

To what extent can our findings explain the dynamical wetting transition for receding water drops? The inverse of Ohnesorge number for water at room temperature is $5 \times 10^{2}$, using the capillary length as the length scale. For the case of water drops with contact angle $70^{\circ}$ (1.2 radian) sliding on a turntable [4], our model indeed predicts a reduction of the critical speed by about a factor 2 . On the other hand, the model predicts almost no inertial effects when the contact angle is $30^{\circ}(0.52$ radian), as was the case for the sliding drops in [1]. A possible reason for this inconsistency is that in the vicinity of the critical speed, the tail of the droplet becomes very sharp, thus the problem cannot be treated as two-dimensional anymore. Alternatively, it has been suggested that other microscopic mechanisms such as thermal activation may be of importance for low-viscosity liquids as water [91]. Clearly, many aspects of the present model can be improved. First, one may attempt a more systematic expansion of the Navier-Stokes equations, in which the assumption of the parabolic profile is no longer needed. In addition, the strong dependence on the contact angle would make it interesting to study large $\theta$, e.g. using numerical simulations. Still, we expect the qualitative features of our model to be valid, just as it was able to capture the influence of inertia on Landau-Levich films [88]. From an experimental point of view, an experimental study of dip-coating with water would provide valuable information to address these issues in further detail. 


\section{7 \\ Summary and Outlook}

The speed at which a liquid can move over a substrate is strongly limited when the surface is partially dry. The wetting dynamics is then governed by the motion of the contact line, which has remained a challenging problem in fluid mechanics. Such moving contact lines play a key role for printing and imaging techniques. The technology of Immersion Lithography, for example, uses a portion of water between a lens and a substrate in order to increase the resolution. When the substrate is moving too fast, droplets are emitted at the receding part of the water. Similarly, air bubbles can be observed in the advancing part, suggesting entrainment of air at the advancing contact line. The transition from a steady interface to drop emission or air entrainment is called a dynamical wetting transition, and originates from the maximum speed at which contact lines can move across a surface. This thesis therefore addresses the fundamentals of "dynamical wetting transitions".

A convenient geometry to study fundamental aspects of dynamical wetting transitions is dip coating, in which a solid is withdrawn from or plunged into a liquid reservoir. This geometry has the advantage that the flow is two-dimensional, which largely simplifies the governing equations. This thesis addresses two types of wetting transitions in a dip coating geometry: film deposition at receding contact lines (withdrawing plate, Chapters 2, 3 and 6), and air entrainment by advancing contact lines (plunging plate, Chapters 4 and 5). For the receding contact line problem, usually the flow of air can be neglected since the air viscosity $\eta_{g}$ is much smaller than the liquid viscosity $\eta_{\ell}$. Thus the relevant parameters are the capillary number, $\mathrm{Ca} \equiv \eta_{\ell} \mathrm{U} / \gamma$, 
which is the ratio between the viscous and capillary forces, and the static contact angle $\theta_{e}$. For the advancing contact line problem, the situation becomes more complicated since usually a recirculation of the air occurs in a very confined region near the contact line. This significantly increases the shear stress in the air. Above a certain flow speed, the dissipation in the air can no longer be neglected. Hence one more parameter has to be introduced to characterize advancing contact line problems, namely the viscosity ratio between the air and the liquid.

We first discuss the dynamical wetting transition at a receding contact line. In Chapter 2, we consider a smooth solid plate being withdrawn from a bath of viscous liquid which it does not wet. The steady state solutions can be numerically computed by the lubrication equation. It is found that there is a maximum speed below which the solid can be withdrawn without leaving a film on the substrate. This critical speed for the dynamical wetting transition arises when the solutions bifurcate into upper branch. The interface profile of the upper branch solution is elongated in the direction of the flow, demonstrating a "finger" shape behind the contact line in small scales. In this study, we add the local analysis of the upper branch of the bifurcation to a previous analysis of the lower branch using matched asymptotic expansion. Due to the "finger" behind the contact line, the outer solution can no longer match the inner solution in the vicinity of the contact line position. We propose that the outer solution should match the inner solution at the "apparent" position of the contact line which is of certain distance (length of the finger) below the real contact line position. It turns out the shift of the contact line position calculated by the matching agrees very well with the numerical solutions of the full lubrication equation. We also show that the bifurcation is of saddle-node type. Thus we have provided a complete description of the dynamical wetting transition in terms of a matched asymptotic expansion.

In Chapter 3, we investigate the effect of the macroscopic geometry on the dynamical wetting transition. Instead of a plate, we consider the withdrawal of a fiber and investigate the role of the fiber radius. First, we use a matched asymptotic expansion to derive the maximum speeds of dewetting for the limiting cases of large fiber radius and small fiber radius. We then develop a modified lubrication equation for flow along a fiber of different radii. The maximum speeds computed by the modified lubrication equation turn out to agree very well with the matched asymptotic expansion. To further investigate the transition we numerically determine the bifurcation diagram for steady menisci. It is found that the meniscus profiles on thick fibers are smooth, even when there is a film deposited between the bath and the contact line, while profiles on thin fibers exhibit strong oscillations. We propose that this may be relevant to experimental observations that transitions occur at zero apparent contact angle for the thin fiber case but not for the thick fiber case. A detailed experimental 
study on the dynamical wetting transition for varying fiber radius is still lacking. In particular it would be interesting to investigate what type of film will be deposited on a thin fiber.

In Chapter 2 and 3, we restrict ourselves to small Reynolds number regimes, namely the inertial terms were neglected. In Chapter 6 we release this restriction since inertia could be important for low viscosity liquids such as water. We study the inertial effect on the dynamical wetting transition, once more by withdrawing a plate from a reservoir of liquid. Based on thin film approximations, we developed a lubrication-type equation which takes the inertial effect into consideration. We find that once inertial terms start to have effect, the critical capillary number is reduced with increasing Reynolds number $R e$. This is because the inertia tends to maintain the flow. In an experiment similar to the Immersion Lithography setup [4], it was previously found that the critical capillary number is a factor two smaller for water than the more viscous silicone oils. Our theoretical results could explain this observation, since for water the inertia is indeed important. The main assumption of our model is that the velocity field is approximated by a parabolic profile. This means we consider a small perturbation from the viscous dominated regime. In the framework of the thin film approximation, the small parameter is $R e h h^{\prime}$, where $h$ is the the liquid thickness and $h^{\prime}$ the slope of the interface. Thus if $R e$ is too large, such that $R e h h^{\prime}$ is no longer small, our model will no longer be quantitatively accurate. Interestingly, for a thin film of water this criterion is very often fulfilled. Experimental verification of our model would provide more understanding of the dynamical wetting transition in less viscous liquids. It remains a great challenge to study wetting dynamics when $R e$ is pushed to much higher values.

Next we consider the case of advancing contact lines. The entrainment of air by advancing contact lines is studied experimentally in Chapter 4 by plunging a solid plate into a reservoir of viscous silicone oil of viscosities $\eta_{\ell}$ ranging from 0.02 to 5 Pa.s. Above an entrainment velocity, we observe the formation of an air film, which is unstable and subsequently decays into bubbles. Unexpectedly, we find that the entrainment speed depends on the liquid viscosity much more weakly than $1 / \eta_{\ell}$. This implies the flow inside the air film plays a crucial role. We explain this result by comparing the dissipation inside the air and the liquid in a wedge geometry. The dissipation in the air increases dramatically as $\eta_{g} /\left(\pi-\theta_{w}\right)$ when the wedge angle $\theta_{w}$ measured in the liquid gets very close to $\pi$, which is what happens at the wetting transition. At the same time, dissipation in the liquid decreases as $\eta_{\ell}\left(\pi-\theta_{w}\right)^{2}$. Hence, even a very small air viscosity could have significant contribution to the total dissipation. During the breakup of the air film, we observed the oil reestablished the contact with the solid and formed the so-called "rewetting bridges". Strikingly, we 
found the bridges spread out in a way similar to the dewetting holes but the roles of air and liquid are exchanged. However, the spreading speed of the "rewetting" bridges is orders of magnitude larger than the dewetting speed.

After this experimental study, we develop a hydrodynamic model in Chapter 5 to study the dynamical wetting transition in two-phase flow, in particular the entrainment of air. In the spirit of the standard lubrication equation for flows in a thin film, we approximate the local flow by flows in a wedge. Once the flow is known, the pressure difference across the interface can be determined, as well as the interface curvature. Using this model, we calculate the meniscus deformations for various values of the capillary number and viscosity ratio $R$. The results are verified by comparing with Lattice Boltzmann simulations. The agreement turns out to be very good in particular for relatively small $\mathrm{Ca}$. In order to study air entrainment, we explore the parameter space of small $R$. Contrary to existing models, we find that the critical speed of air entrainment does not scale as $1 / \eta_{\ell}$, but has a much weaker dependence. This is consistent with the experiments discussed in Chapter 4, and our model further quantifies the influence of the air. Since we approximate the flow by flows in a wedge, our model is limited to small interface curvature, and thus to small values of $\mathrm{Ca}$. Modeling for large Ca remains a challenge. Another issue that remains ambiguous is the physical mechanisms for the cutoff at the contact line. We propose that in the air phase, the slip length is determined by the mean free path of the gas. This provides a new perspective on experiments where the air pressure is reduced, which gives rise to a remarkable increase in entrainment speeds [23]. The interesting point is that depressurizing the air will increase the mean free path. Interpreting the pressure reduction as an increase of the slip length, our model indeed predicts an increase of the critical speed that is comparable to the experimental observation. More studies have to be carried out to provide a definite answer to these questions. 


\section{References}

[1] T. Podgorski, J. M. Flesselles, and L. Limat, "Corners, cusps, and pearls in running drops”, Phys. Rev. Lett. 87, 036102(1)-(4) (2001).

[2] T. D. Blake and K. J. Ruschak, "A maximum speed of wetting.", Nature 282, 489-491 (1979).

[3] J. Eggers, "Hydrodynamic theory of forced dewetting", Phys. Rev. Lett. 93, 094502 (2004).

[4] K. G. Winkels, I. R. Peters, F. Evangelista, M. Riepen, A. Daerr, L. Limat, and J. Snoeijer, "Receding contact lines: From sliding drops to immersion lithography”, Eur. Phys. J. Special Topics 192, 195 (2011).

[5] J. H. Burnett, S. G. Kaplana, E. L. Shirleya, P. Tompkins, and J. E. Webb, Proceedings of SPIE 5754, Optical Microlithography XVIII (edited by B. W. Smith, SPIE, Bellingham, WA, 2005, Vol. 5754).

[6] C. Huh and L. E. Scriven, "Hydrodynamic model of steady movement of a solid/liquid/fluid contact line", J. Coll. Int. Sci. 35, 85-101 (1971).

[7] H. Hervet and P.-G. de Gennes, "The dynamics of wetting: Precursor films in the wetting of "dry" solids", C. R. Acad. Sc. Paris, Serie II 299, 499-503 (1984).

[8] P. Seppecher, "Moving contact lines in the cahn-hilliard theory", Int. J. Engng. Sci. 34, 977-992 (1996).

[9] D. Bonn, J. Eggers, J. Indekeu, J. Meunier, and E. Rolley, "Wetting and spreading", Rev. Mod. Phys. 81, 739 (2009).

[10] A. Oron, S. H. Davis, and S. G. Bankoff, "Long-scale evolution of thin liquid films", Rev. Mod. Phys. 69, 931-980 (1997).

[11] J. Eggers, "Existence of receding and advancing contact lines", Phys. Fluids 17, 082106 (2005).

[12] J. H. Snoeijer, G. Delon, M. Fermigier, and B. Andreotti, "Avoided critical behavior in dynamically forced wetting”, Phys. Rev. Lett. 96, 174504 (2006).

[13] J. H. Snoeijer, B. Andreotti, G. Delon, and M. Fermigier, "Relaxation of a dewetting contact line part 1: A full-scale hydrodynamic calculation", J. Fluid Mech. 579, 63 (2007). 
[14] G. Delon, M. Fermigier, J. H. Snoeijer, and B. Andreotti, "Relaxation of a dewetting contact line part 2: Experiments", J. Fluid Mech. 604, 55 (2008).

[15] J. H. Snoeijer, J. Ziegler, B. Andreotti, M. Fermigier, and J. Eggers, "Thick films coating a plate withdrawn from a bath”, Phys. Rev. Lett. 100, 244502 (2008).

[16] J. Ziegler, J. H. Snoeijer, and J. Eggers, "Film transitions of receding contact lines”, Eur. Phys. J. Special Topics 166, 177 (2009).

[17] L. D. Landau and B. V. Levich, "Dragging of a liquid by a moving plate", Acta physico-chimica USSR 17, 42 (1942).

[18] R. V. Sedev and J. G. Petrov, "The critical condition for transition from steady wetting to film entrainment", Colloids and Surfaces 53, 147 (1991).

[19] E. Lorenceau, F. Restagno, and D. Quéré, "Fracture of a viscous liquid", Phys. Rev. Lett. 90, 184501 (2003).

[20] J. Eggers, "Air entrainment through free-surface cusps", Phys. Rev. Lett. 86, 4290 (2001).

[21] R. G. Cox, "The dynamics of the spreading of liquids on a solid surface. part 1. viscous flow", J. Fluid Mech. 168, 169-194 (1986).

[22] L. Xu, W. W. Zhang, and S. R. Nagel, "Drop splashing on a dry smooth surface", Phys. Rev. Lett. 94, 184505 (2005).

[23] H. Benkreira and M. I. Khan, "Air entrainment in dip coating under reduced air pressures”, Chem. Eng. Sci. 63, 448-459 (2008).

[24] B. V. Deryaguin and S. M. Levi, Film Coating Theory (Focal, London) (1964).

[25] D. Quéré, "Fluid coating on a fiber", Annu Rev. Fluid Mech. 31, 347 (1999).

[26] S. J. Weinstein and K. J. Ruschak, "Coating flows", Annu. Rev. Fluid Mech. 36, 29-53 (2004).

[27] O. V. Voinov, "Hydrodynamics of wetting [english translation]", Fluid Dynamics 11, 714-721 (1976).

[28] T. S. Chan, T. Gueudré, and J. Snoeijer, "Maximum speed of dewetting on a fiber", Phys. Fluids 23, 112103 (2011). 
[29] J. H. Snoeijer, "Free surface flows with large slopes: beyond lubrication theory”, Phys. Fluids 18, 021701 (2006).

[30] L. D. Landau and E. M. Lifshitz, Fluid Mechanics (Pergamon: Oxford) (1984).

[31] Drazin, Nonlinear systems (Cambridge University Press, Cambridge) (1992).

[32] B. R. Duffy and S. K. Wilson, "A third-order differential equation arising in thin-film flows and relevant to tanner's law", Appl. Math. Lett. 10, 63 (1997).

[33] M. Abramowitz and I. A. Stegun, Handbook of Mathematical Functions (Dover) (1968).

[34] B. V. Deryaguin, "On the thickness of a layer of liquid remaining on the walls of vessels after their emptying, and the theory of the application of photoemulsion after coating on the cine film”, Acta physico-chimica USSR 20, 349 (1943).

[35] P.-G. de Gennes, "Deposition of langmuir-blodget layers", Coll. Pol. Sci. 264, 463-465 (1986).

[36] L. W. Schwartz, D. Roux, and J. J. Cooper-White, "On the shapes of droplets that are sliding on a vertical wall”, Physica D 209, 236 (2005).

[37] D. F. James, "The meniscus on the outside of a small circular cylinder", J. Fluid Mech. 63, 657 (1973).

[38] M. Maleki, E. Reyssat, D. Quéré, and R. Golestanian, "On the landau-levich transition", Langmuir 23, 10116 (2007).

[39] C. Duez, C. Ybert, C. Clanet, and L. Bocquet, "Making a splash with water repellency”, Nat. Phys. 3, 180 (2007).

[40] J. H. Burnett, S. G. Kaplana, E. L. Shirleya, P. Tompkins, and J. E. Webb, "High-index materials for $193 \mathrm{~nm}$ immersion lithography", Proceedings of SPIE 5754, Optical Microlithography XVIII 5754 (2005).

[41] U. Thiele, K. Neuffer, M. Bestehorn, Y. Pomeau, and M. G. Velarde, "Sliding drops on an inclined plane", Colloid Surf. A 206, 87-104 (2002).

[42] L. Limat and H. A. Stone, "Three-dimensional lubrication model of a contact line corner singularity”, Europhys. Lett. 65, 365 (2004).

[43] N. Le Grand, A. Daerr, and L. Limat, "Shape and motion of drops sliding down an inclined plane", J. Fluid Mech. 541, 293-315 (2005). 
[44] J. Snoeijer, N. Le Grand, L. Limat, H. A. Stone, and J. Eggers, "Cornered drop and rivulets", Phys. Fluids 19, 042104 (2007).

[45] L. M. Hocking, "The spreading of a thin drop by gravity and capillarity", Q. J. Mech. Appl. Math. 36, 55-69 (1983).

[46] E. Lauga, M. P. Brenner, and H. A. Stone, "Microfluidics: the no-slip boundary condition", in Springer Handbook of Experimental Fluid Mechnaics, edited by C. Tropea, J. F. Foss, and A. Yarin, 1219-1240 (Springer) (2008).

[47] C. Cottin-Bizonne, B. Cross, A. Steinberger, and E. Charlaix, "Boundary slip on smooth hydrophobic surfaces: intrinsic effets and possible artifacts", Phys. Rev. Lett. 94, 056102 (2005).

[48] R. Golestanian and E. Raphaël, "Roughening transition in a moving contact line", Phys. Rev. E 67, 031603 (2003).

[49] J.-F. Joanny and P.-G. de Gennes, "Competition between wetting and adverse macroscopic forces [in french]”, C. R. Acad. Sc. Paris, Serie II 299, 605-608 (1984).

[50] R. Golestanian and E. Raphaël, "Relaxation of a moving contact line and the landau-levich effect", Europhys. Lett. 55, 228-234 (2001).

[51] C. Redon, F. Brochard-Wyart, and F. Rondelez, "Dynamics of dewetting", Phys. Rev. Lett. 66, 715 (1991).

[52] H. N. Oguz, A. Prosperetti, and A. Kolaini, "Air entrapment by a falling water mass”, J. Fluid Mech. 294, 181-207 (1995).

[53] E. Lorenceau, D. Quéré, and J. Eggers, “Air entrainment by a viscous jet plunging into a bath”, Phys. Rev. Lett. 93, 254501 (2004).

[54] J. de Jong, R. Jeurissen, H. Borel, M. van den Berg, H. Wijshoff, H. Reinten, M. Versluis, A. Prosperetti, and D. Lohse, "Entrapped air bubbles in piezodriven inkjet printing: Their effect on the droplet velocity", Phys. Fluids 18, 121511 (2006).

[55] D. D. Joseph, J. Nelson, M. Renardy, and Y. Renardy, "Two-dimensional cusped interfaces”, J. Fluid Mech. 223, 383-409 (1991).

[56] J.-T. Jeong and H. K. Moffatt, "Free-surface cusps associated with a flow at low reynolds numbers", J. Fluid Mech. 241, 1-22 (1992). 
[57] M. M. Driscoll, C. C. Stevens, and S. R. Nagel, "Thin film formation during splashing of viscous liquids", Phys. Rev. E 82, 036302 (2010).

[58] P. Tsai, R. C. A. van der Veen, M. van de Raa, and D. Lohse, "How micropatterns and air pressure affect splashing on surfaces", Langmuir 26, 16090 (2010).

[59] C. Josserand, P. Ray, and S. Zaleski, "Air entrapment and splashing treshold in drop impacts", 7th International Conference on Multiphase Flow (2010).

[60] S. Mandre, M. Mani, and M. P. Brenner, "Precursors to splashing of liquid droplets on a solid surface", Phys. Rev. Lett. 102, 134502 (2009).

[61] M. Mani, S. Mandre, and M. Brenner, "Events before droplet splashing on a solid surface”, J. Fluid Mech. 647, 163 (2010).

[62] R. Ledesma-Aguilar, R. Nistal, A. Hernández-Machado, and I. Pagonabarraga, "Controlled drop emission by wetting properties in driven liquid filaments", Nature Materials 10, 367-371 (2011).

[63] R. Burley and B. S. Kennedy, "An experimental study of air entrainment at a solid/liquid/gas interface”, Chem. Eng. Sci. 31, 901-911 (1976).

[64] P. G. Simpkins and V. J. Kuck, "Air entrapment in coatings by way of a tipstreaming meniscus", Nature 403, 641-643 (2000).

[65] H. Benkreira and J. B. Ikin, "Dynamic wetting and gas viscosity effects", Chem. Eng. Sci. 65, 1790-1796 (2010).

[66] J. C. Flitton and J. R. King, "Surface-tension-driven dewetting of newtonian and power-law fluids", J. Engin. Math. 50, 241-266 (2004).

[67] J. H. Snoeijer and J. Eggers, "Asymptotics of the dewetting rim", Phys. Rev. E 82, 056314 (2010).

[68] G. Debregeas and F. Brochard-Wyart, "Nucleation radius and growth of a liquid meniscus”, J. Colloid Interface Sci. 190, 134 (1997).

[69] R. Burley and B. S. Kennedy, "A study of the dynamic wetting behavior of polyester tapes", Brit. Polym. J. 8, 140-143 (1976).

[70] R. W. Hewson, "Free surface model derived from the analytical solution of stokes flow in a wedge.”, J. Fluids Engineering 131, 041205 (2009). 
[71] E. Lemmon and R. T. Jacobsen, "Viscosity and thermal conductivity equations for nitrogen, oxygen, argon, and air", Int. J. Thermophys. 25, 21 (2004).

[72] L. Bocquet, "Slipping of a fluid on a surface of controlled roughness", C. R. Acad. Sci. Paris 316, serie 2, 7 (1993).

[73] M. K. Andrew and P. D. harris, "Damping and gas viscosity measurements using a microstructure", Sensors and Actuators A 49, 103-108 (1995).

[74] T. D. Blake and K. J. Ruschak, "Wetting: static and dynamic contact lines", in Liquid film coating, edited by S. F. Kistler and P. M. Schweizer (London, Chapman and Hall) (1997).

[75] D. Quéré, "On the minimal velocity of forced spreading in partial wetting (in french)", C. R. Acad. Sci. Paris, Serie II 313, 313-318 (1991).

[76] A. Marchand, T. S. Chan, J. H. Snoeijer, and B. Andreotti, "Air entrainment by contact lines of a solid plate plunged into a viscous fluid", Phys. Rev. Lett. 108, 204501 (2012).

[77] J. Eggers, "Toward a description of contact line motion at higher capillary numbers", Phys. Fluids 16, 3491 (2004).

[78] R. Buckingham, M. Shearer, and A. Bertozzi, "Thin film traveling waves and the navier slip condition”, SIAM J. Appl. Math. 63, 722-744 (2003).

[79] P. L. Bhatnagar, E. P. Gross, and M. Krook, "A Model for Collision Processes in Gases. I. Small Amplitude Processes in Charged and Neutral One-Component Systems", Phys. Rev. 511-525 (1954).

[80] S. Succi, Lattice Boltzmann equation for fluid dynamics and beyond (Oxford University press) (2001).

[81] D. A. Wolf-gladrow, Lattice-Gas Cellular Automata and Lattice Boltzmann Models - An Introduction (Springer) (2005).

[82] X. Shan and G. Doolen, "Multicomponent lattice-Boltzmann model with interparticle interaction", Journal of Statistical Physics 81, 379-393 (1995).

[83] X. Shan and H. Chen, "Lattice Boltzmann model for simulating flows with multiple phases and components”, Phys. Rev. E 47, 1815 (1993).

[84] X. Shan, "Simulation of nonideal gases and liquid-gas phase transitions by the lattice Boltzmann equation", Phys. Rev. E 49, 2941-2948 (1994). 
[85] R. Benzi, L. Biferale, M. Sbragaglia, S. Succi, and F. Toschi, "Mesoscopic modeling of a two-phase flow in the presence of boundaries: The contact angle", Phys. Rev. E 74, 021509 (2006).

[86] H. Huang, J. D. T. Thorne, M. G. Schaap, and M. C. Sukop, "Proposed approximation for contact angles in shan-and-chen-type multicomponent multiphase lattice boltzmann models", Phys. Rev. E 76, 066701 (2007).

[87] E. Rame and S. Garoff, "Microscopic and macroscopic dynamic interface shapes and the interpretation of dynamic contact angles", J. Colloid Interface Sci. 177, 234-244 (1996).

[88] A. Koulago, V. Shkadov, D. Quéré, and A. de Ryck, "Film entrained by a fiber quickly drawn out of a liquid bath", Phys. Fluids 7, 1221 (1995).

[89] L. Prandtl, "Über flüssigkeitsbewegung bei sehr kleiner reibung", in Ludwig Prandtl, Gesammelte Abhandlungen, Vol. II, edited by W. Tollmien, H. Schlichting, and H. Görtler, 575-584 (Springer) (1961).

[90] R. G. Cox, "Inertial and viscous effects on dynamic contact angles", J. Fluid Mech. 357, 249-278 (1998).

[91] D. Seveno, A. Vaillant, R. Rioboo, H. A. o, J. Conti, and J. D. Coninck, "Dynamics of wetting revisited", Langmuir 25, 1303413044 (2009). 


\section{Samenvatting}

De snelheid waarmee een vloeistofdruppel zich verplaatst over een oppervlak wordt bepaald door het krachtenspel aan de rand van de druppel op het substraat. De dynamica van deze rand, de "contactlijn", is een fundamenteel probleem in de vloeistoffysica. De contactlijn speelt een belangrijke rol bij diverse moderne industriële toepassingen. Bijvoorbeeld, in Immersie Lithografie wordt een laagje water gebruikt tussen de lens en het substraat. Dit heeft als doel de optische resolutie te vergroten waarmee structuren kunnen worden afgebeeld op een silicium chip. Echter, wanneer het substraat te snel wordt gescand zullen er kleine druppeltjes achterblijven of luchtbellen worden ingevangen. Deze overgangen waarbij druppels of bellen ontstaan heten "dynamische bevochtigingstransities" en vormen het onderwerp van dit proefschrift.

Een geschikte methode om de dynamica van bevochtiging te onderzoeken is "dip coating", waarbij een substraat in een vloeistofbad wordt gedompeld, of eruit wordt getrokken. In het eerste geval is de beweging van de contactlijn voortschrijdend over het substraat (bevochtiging), terwijl in het tweede geval de contactlijn zich terugtrekt (ontvochtiging). De stroming in de vloeistof die in deze geometrie ontstaat is voor beide gevallen tweedimensionaal, waardoor de onderliggende wiskundige vergelijkingen eenvoudiger op te lossen zijn. Dit proefschrift onderzoekt twee verschillende bevochtigingstransities: depositie van vloeistoffilms door terugtrekkende contactlijnen (Hoofdstukken 2, 3 en 6)), en inhappen van lucht voor het geval van voortschrijdende contactlijnen (Hoofdstukken 4 en 5). Voor terugtrekkende contactlijnen kan de stroming in de lucht worden verwaarloosd, omdat de viscositeit van lucht $\eta_{g}$ veel kleiner is de de viscositeit van vloeistoffen $\eta_{\ell}$. De relevante kentallen zijn het capillaire getal, $\mathrm{Ca} \equiv \eta_{\ell} \mathrm{U} / \gamma$, de verhouding van viskeuze en capillaire krachten, en de evenwichtscontacthoek $\theta_{e}$. Echter, voor het geval van voorschrijdende contactlijnen, waarbij luchtbellen worden ingehapt, is de luchtstroming wel van belang. In dat geval is er een extra kental van belang, namelijk de verhouding van viscositeiten $\eta_{g} / \eta_{\ell}$.

In Hoofdstuk 2 bestuderen we terugtrekkende contactlijnen door een substraat met constante snelheid uit een vloeistofreservoir te trekken. Met behulp van numerieke berekeningen worden eerst alle "steady-state" oplossingen gevonden, waarbij we gebruik maken van de lubricatiebenadering. Het blijkt dat er een maximale 
snelheid is waarmee een contactlijn over een substraat kan bewegen. Wanneer het substraat met een hogere snelheid uit het bad wordt gehaald zal deze een vloeistoflaag meetrekken, en dus bevochtigd worden. Het optreden van de maximumsnelheid kan in detail worden verklaard met behulp van een asymptotische expansie van de lubricatievergelijkingen. Met deze techniek tonen we aan dat de bevochtigingstransitie optreedt door een bifurcatie, waarbij een stabiele en instabiele tak van oplossingen samenkomen. Ook vinden we een analytische voorspelling voor de maximumsnelheid. Vervolgens onderzoeken we in Hoofdstuk 3 in hoeverre de bevochtigingstransitie afhangt van de geometrie van de stroming. In plaats van een vlak substraat bekijken we daarom de bevochtigingstransitie voor cilinders met verschillende doorsnedes. Onze berekeningen laten zien dat de maximumsnelheid toeneemt bij afnemende cilinderradius. Een opvallende voorspelling van onze theorie is dat voor dunne cilinders, de vloeistoffilm die wordt meegetrokken niet mooi vlak kan blijven. In tegenstelling tot de film op vlakke substraten vinden we sterke oscillaties van de dikte van de vloeistoflaag. Het zou interessant zijn om experimenteel te onderzoeken of dergelijke vloeistoflagen stabiel zijn, of uiteenvallen in kleine druppeltjes.

Bovenstaande resultaten gelden voor sterk viskeuze vloeistoffen, waarvoor de massatraagheid van de vloeistof kan worden verwaarloosd. Traagheid is echter wel degelijk van belang voor bevochtiging door water. In Hoofdstuk 6 ontwikkelen we daarom een model waarmee het effect van traagheid kan worden onderzocht. We vinden dat de maximumsnelheid van de contactlijn afneemt door het effect van traagheid. De afname die wordt voorspeld door het model komt goed overeen met recente experimenten in de context van Immersie Lithografie. Een meer gedetailleerde vergelijking met dip-coating experimenten zal echter nodig zijn om het model, en de onderliggende aannames, te valideren.

Hoofdstuk 4 beschrijft een experimentele studie naar het inhappen van lucht door voortschrijdende contactlijnen. Het substraat wordt gedompeld in reservoirs gevuld met siliconenoliën van verschillende viscositeit $\eta_{\ell}$. Voor elke olie bepalen we de kritische snelheid waarboven een luchtlaag wordt meegetrokken in de vloeistof. Deze luchtlaag breekt later op in de vorm van luchtbellen in de olie. We vinden dat de kritische snelheid afneemt bij toenemende viscositeit. Echter, het verrassende resultaat is dat deze afname veel minder sterk is dan $\sim 1 / \eta_{\ell}$, zoals de klassieke theoriën voorspellen. Dit duidt erop dat de stroming in de luchtlaag een sterke invloed heeft op het proces.

Deze hypothese wordt verder ondersteund door de theoretische analyse van Hoofdstuk 5. In dit hoofdstuk presenteren we een uitbreiding van de lubricatietheorie, waarbij de krachten uitgeoefend door de luchtstroming worden berekend. Deze krachten hebben inderdaad een sterke invloed op de vorm van de meniscus wanneer de lokale 
contacthoek bijna $180^{\circ}$ is, zoals bij het inhappen van lucht. Onze theoretische voorspellingen zijn consistent met de experimentele resultaten. Het blijft echter een uitdaging om een volledig kwantitatieve beschrijving te vinden wanneer het capillaire getal Ca niet klein is. Tot slot biedt ons model een nieuw perspectief op experimenten in de groep van Benkreira, die gedaan zijn onder een sterk gereduceerde luchtdruk. Het verlagen van de druk leidde tot een verrassende toename van de kritische snelheid voor het inhappen van lucht. Het verlagen van de druk leidt ook tot een toename van de vrije weglengte van de gasmoleculen. Wanneer we de vrije weglengte interpreteren als de sliplengte van het gas aan de wand kunnen we met ons model deze verrassende experimenten verklaren. 


\section{Acknowledgements}

Time runs fast. It has been four years since the first time I stepped on the land of this country of windmills (my impression of the Netherlands before I came). Living in a country far away from home is always not easy, especially in a culture completely different from what I am familiar with . Very luckily, thanks to all the friends I have met here and all the wonderful group members and staff in POF, this four years has become one of the greatest time in my life. When I was away for some time for conferences or meeting, it happened a few times that I started to miss Enschede, just like missing my home!

First, I would like to thank Detlef and Jacco for giving me the opportunity to do my $\mathrm{PhD}$ in POF. I did experiments during my master in Hong Kong. Then I decided to work on theory for my PhD study. Perhaps in Europe it is not uncommon to switch between experimental and theoretical work when one starts a $\mathrm{PhD}$, but for me, it was a big change. I appreciate Detlef and Jacco a lot for trusting me and letting me make this change.

The first time I talked to Jacco was on the phone. My English was bad, indeed most of the time I did not understand what he was talking about in spite of his excellent English speaking. I also tried to explain what I had been working on and obviously, I did not succeed. Back then I had the impression that, yes, Jacco was difficult to communicate with, haha! After the phone chat, I came to visit POF. I met Jacco and we had a conversation face to face. This time the process of knowledge exchange was better. Well, Jacco seemed to be a nice guy! I started to work in POF in September 2008, a few months after my visit. It was a new environment to me, there were many things I had to adapt to. Not just the food and the weather, but also the way people do research and communicate with each other. Jacco was always very patient explaining everything to me. I still remember he said to me when I had just arrived: "We work very slowly ;-)". So I always enjoyed working in POF. Talking to Jacco is always a great pleasure. I enjoyed every moment we talked about science, not only the deep insights and ideas he has come up with and the skills I have learnt, but also the atmosphere felt like talking to an old friend. He always encourages you and you are never afraid of making mistakes, and then you build up confidence. Jacco, with 
your guidance, this 4-year period working in POF has been a fantastic experience for me. I could never imagine a better advisor than you. I thank you deep from my heart! I would also like to thank Leon and Danielle. I enjoyed very much playing with you, Leon. And Danielle, the pasta you prepared was really great!

I was very lucky to have the opportunity to work with many nice people. In the third year of my PhD, I went to work in Bruno Andreotti's group in Paris for two months. It was pretty difficult to find a place to stay at that period of time and we were in a bit of a hurry. I know Bruno had searched many websites, contacted many landlords, and spent a lot of time trying to get a comfortable apartment for me. In the end, after all that effort, the apartment I stayed in was wonderful. During my visit and afterward as well, we had frequent discussions. I learnt a lot from him and I hope we will continue to interact. Thanks a lot, Bruno. I also worked with Antonin Marchand in the lab in Paris on the air entrainment problem. Antonin is an excellent experimentalist. I was always amazed by his skill and idea of constructing a nice setup for measurements. We obtained many nice results. Antonin, thanks! I would also like to thank Thomas for working together on the withdrawing fiber problem, it was amazing that you could manage the tough matched asymptotic expansion in just a very short period of time. Sudhir, I am very happy we had so many nice discussions on the air entrainment models. I am looking forward to finish our paper.

Chao was my daily advisor when I was doing my master's degree in Hong Kong. As many of us have experienced, working with Chao is fruitful, but also big fun. I have learnt a lot from him. He always had many great ideas, in particular on performing a nice experiment. Chao joined POF one year earlier than I did. Because of Chao, adapting to a new life in Netherlands has been much easier. And I feel like I am living at home, and he is my older brother, giving me support and encouragement. Thanks a lot, Chao! And of course Vivian also, I love all the Chinese food you prepare!

Working in POF is enjoyable because of all the friendly and active members, and a very comfortable environment. POF is a fantastic research group. Whenever I had problems with my computer, I came to Bas, and all problems were solved. Thanks Bas! We are all happy to see POF getting bigger and bigger, and we are all amazed that Detlef manages this huge group so well. On the same level of importance, I would say, there is another person who has to deal with problems from all of us and is doing an amazing job. Joanita, thanks a lot! Koen and I work in similar projects. It was a great time every time we had discussions about different physical problems, went to conferences and workshops, and made posters together. Talking to Koen is always pleasant. No matter what the subject is, he is always willing to listen and expresses his interest. Thanks Koen. Our office room may not be the most active one, but every time we had conversations, it was fun. Thanks Bram, ShangJiong, Todd, 
Stefan, Yoshi and Tuan. Doing research in POF is great, and playing football for POF is also great. Even though we did not win every game (or we lost quite many of them), I enjoyed playing with all of you every time. Many thanks to captains Stefan and Arjan for managing our POF football team. I also give special thanks to Sylvain and Koen, I greatly appreciated your vision, you were always able to notice my runs and gave amazing passes. Koen, it was a pity you did not play so often, otherwise we would have won more matches! One great advantage of working in a big group is that you can interact with many people and have lot of fun. Here I would like to thank Ceyda, Raja, Dennis, Oscar, Vivek, Sander, Federico, Claas, Henry, Antonin, Hanneke, Wilco, Ivo, Amy, Bram, Alvaro, Julian, Devaraj, Michel and many others for many nice conversations, both face to face and on Facebook, at movie nights and indoor football playground, etc.

I would also like to express my gratitude to ASML, Marie Curie Actions, FOM and Océ for funding this project. In particular, I would like to thank Michel Riepen, Ramin Badie and Pieter de Witte for many discussions and organizing many interesting meetings.

Apart from all the group members in POF, life in this four years would not have been so beautiful without all the other friends in Enschede. I still find it incredible when I met DanChao, ShaoLin and Sheng on the streets in the first week I arrived Enschede. It turned out we were living in the same building called Macandra, and gradually we became very good friends. Some time later, HaiShan, XinFu and CuiYang joined us. And we even had a name for our group "Macandra the sixth floor". I will never forget the times we prepared dinner together every day, traveled to different places, played card games, ping-pong...... and had parties with many friends. Whatever we did, it was always part of the happiest moments in these four years. DanChao, I always think the trip cycling from Enschede to Amsterdam was really amazing and unforgettable. Your little child is very big now, and very lovely! ShaoLin, I wish you enjoy life in Amsterdam with ZiZhen. Sheng, is your business running well? HaiShan, I wish you a successful academic career. XinFu and Cui Yang, I will always miss the amazing food you prepare.

Two and a half years after I started, I moved to a new apartment where I met my roomates XiaoChen, JiangLai and later YiHan and Qiao Yu. Many thanks to XiaoChen for letting me know about the room. I enjoyed every time we had big dinner parties with many friends in our cosy, well-decorated sitting room. I specially thank YiHan, Ying Ying, Rena and XiaoChen for organizing so many nice gatherings and parties. I would also like to thank TaoYin, XiuLi, Leo, WangZhao and many others for sharing your great time with me.

Finally, I would like to thank my family for your love and support. This can never 
be described in words. I will be away for a few more years, then I will be back home!

Tak Shing Chan

Enschede, the Netherlands

August 2012 


\section{About the author}

Tak Shing Chan was born on February $6^{\text {th }} 1982$ in Guang Dong, China. He moved to Hong Kong when he was six years old and then grew up there. He went to the Queen Elizabeth school in the first five years of his secondary level study. Then he spent another two years at the Po Leung Kuk Vicwood K.T. Chong Sixth Form College. After graduating from the college in 2002, he continued his study at the Chinese University of Hong Kong and majored in physics. He obtained his Bachelor degree in 2005, after which he joined the turbulence group for his master study under the supervision of Prof. Ke-Qing Xia. In 2008 he joined the Physics of Fluids group at the University of Twente, where he did a PhD on contact line dynamics with Jacco Snoeijer and Detlef Lohse. During these four years, he went twice to Bruno Andreotti's group at the ESPCI in Paris, to perform experiments on air entrainment. He has also attended a number of summer schools and conferences held in different countries in Europe and the USA.

Tak Shing has a wide variety of interests besides physics. He loves traveling and knowing different cultures. During the four years of his $\mathrm{PhD}$, he has visited more than twenty European countries. He likes different sport activities, in particular he is a big fan of football, both playing and watching. Although he rarely sings, he likes musics and dance. He has taken some salsa courses in Enschede, although only managed to dance the first few basic steps. Hopefully, he will continue to learn and practice it when he will be traveling in Cuba. Also painting is something he enjoys a lot. 
